\title{
Flora bromeliolóGica del eStAdo de GuerRero, MÉXICO: RIQUEZA Y DISTRIBUCIÓN
}

\author{
Valeria Angélica Pulido-Esparza, Ana Rosa López-Ferrari y Adolfo Espejo-Serna ${ }^{1}$ \\ Herbario Metropolitano, Departamento de Biología, C.B.S., Universidad Autónoma \\ Metropolitana. Unidad Iztapalapa, Apartado Postal 55-535, 09340 México, D. F. \\ ${ }^{1}$ Autor para la correspondencia. Correo-e: aes@xanum.uam.mx
}

\begin{abstract}
Resumen: El estado de Guerrero resulta de especial interés por la singularidad de su flora bromeliológica, la cual presenta un alto porcentaje de endemitas. Con base en el estudio de 1,250 ejemplares herborizados correspondientes a 797 recolecciones y una detallada revisión bibliográfica, concluimos que la flora bromeliológica hasta ahora conocida para Guerrero consta de 88 especies y 11 géneros. Se registran por primera vez los géneros Racinaea y Ursulaea y las especies Catopsis berteroniana, Hechtia reflexa, Racinaea ghiesbreghtii, Tillandsia andrieuxii, T. gymnobotrya, T. magnusiana, T. marabascoensis, T. polystachia, T. rettigiana, T. tricolor y Ursulaea tuitensis. Los estados con una flora bromeliológica más parecida a la de Guerrero son Jalisco, Michoacán y Colima, seguidos por el Estado de México y Morelos. De los tipos de vegetación que prosperan en el estado, el que presenta una mayor riqueza bromeliológica es el bosque de Quercus con 42 taxa, seguido por los bosques tropical caducifolio con 33 y tropical subcaducifolio con 27. El tipo de vegetación con menor número de especies es el bosque de Juniperus. Se presenta un catálogo completo de la familia, incluyendo claves de identificación a nivel genérico y específico, y se hace un análisis de la representación de la familia en el estado, documentándose detalladamente la distribución de sus especies. Palabras clave: Bromeliaceae, endemismo, florística, Guerrero, México.
\end{abstract}

\begin{abstract}
The state of Guerrero is interesting because of its rich and peculiar Bromeliad flora that includes a large number of endemic species. Based on the study of 1,250 herbarium specimens corresponding to 797 collections, and a detailed bibliographic revision we conclude that the Bromeliad flora known up to now for Guerrero comprises 88 species and 11 genera. The genera Racinaea and Ursulaea and the species Catopsis berteroniana, Hechtia reflexa, Racinaea ghiesbreghtii, Tillandsia andrieuxii, T. gymnobotrya, T. magnusiana, T. marabascoensis, T. polystachia, T. rettigiana, T. tricolor and Ursulaea tuitensis, are recorded for the first time for Guerrero. The states whose Bromeliad flora is most similar to that of Guerrero are Jalisco, Michoacán and Colima, followed by State of México and Morelos. Among the vegetation types occurring in the state, Quercus forest is the richest with 42 taxa, followed by tropical deciduous forest with 33 and tropical subdeciduous forest with 27 . The vegetation type with the lowest species number is the Juniperus forest. Finally, an updated catalogue of the Bromeliaceae from the state of Guerrero, including keys to genus and species identification, is presented; also, the species representation and distribution are analized in detail.
\end{abstract}

Key words: Bromeliaceae, endemism, floristics, Guerrero, Mexico.

$\mathbf{L}$

a familia Bromeliaceae agrupa a nivel mundial cerca de 2,700 especies (Benzig, 2000), de las cuales 342 están representadas en México (Espejo et al, 2004), ocupando el tercer lugar en importancia dentro de las Liliopsidae nativas del país. Además de ser uno de los principales componentes epifíticos y saxícolas de variadas comunidades vegetales del país (Rzedowski, 1978; Aguirre-León, 1992; Wolf y Flamenco, 2003), la familia está ampliamente distribuida en toda la República. De acuerdo con Benzing (2000), las bromeliáceas ejemplifican la radiación adaptativa llevada al extremo y aportan una gran cantidad de servicios a la biota con la que se asocian, desde los detritívoros hasta los polinizadores, por lo que su importancia en los ecosistemas que habitan es de primordial interés.

Además, muchos de los taxa de la familia son valiosos desde un punto de vista económico, ya que son explotados y comercializados a nivel local o incluso nacional para diversos fines, como fuente de obtención de fibras, como alimento, forraje, combustible, medicina o como elementos ornamentales o ceremoniales (Kirby, 1963; Clark, 1965; Gardner, 1982; Brücher, 1989; Martínez, 1990; Ríos y Khan, 1998; Pierce, 2000; Guess y Guess, 2001; Espejo y López-Ferrari, 2003).

A pesar de su importancia y representación en nuestro 
país, las bromeliáceas siguen siendo un grupo poco atendido y entendido por los botánicos mexicanos. Desde la contribución de Smith y Downs para Flora Neotropica (1974, 1977, 1979), no se ha trabajado de forma integral con la familia para México, aunque se cuenta con algunos trabajos florísticos regionales (McVaugh, 1989; Utley y BurtUtley, 1994; Victoria, 2001).

La revisión sistemática de las colecciones de la familia en diversos herbarios nacionales y extranjeros con el fin de completar las Bromeliaceae de México y la constante incorporación de material recolectado en diversos puntos del país, han generado información sobre la representación que tiene el grupo a nivel nacional (Espejo et al., 2004). Entre los estados que sobresalen por su riqueza y su diversidad bromeliológica se encuentran Oaxaca, Chiapas, Veracruz, Jalisco y Guerrero. Este último en particular resulta de especial interés por la singularidad de su flora, la cual presenta el más alto porcentaje de especies endémicas (Espejo y López-Ferrari, 1998). Sin embargo, no existe actualmente un catálogo completo de sus especies, aunque varias de ellas han sido referidas en diversos trabajos de índole florística (Fonseca y Lozada, 1993, Diego y Lozada, 1994; Lozada, 1994; Gual, 1995; Peralta-Gómez, 1995; Verduzco y Rodríguez, 1995; Gallardo, 1996; Vargas y Pérez, 1996; Diego-Pérez, 2000; Diego-Pérez et al., 2001; Fonseca et al., 2001; Lozada et al., 2003; VelázquezMontes y Domínguez-Licona, 2003). Presentamos este trabajo con el triple propósito de completar el catálogo florístico de la familia para el estado, de estimar la representa- tividad de cada taxon en el mismo y de presentar en detalle su distribución espacial conocida.

\section{Área de estudio}

El estado de Guerrero está situado en el sur de la República Mexicana, entre $16^{\circ} 19^{\prime}$ y $18^{\circ} 53^{\prime}$ de latitud norte y entre $98^{\circ} 00^{\prime}$ y $102^{\circ} 11^{\prime}$ de longitud oeste (figura 1 ). Con una extensión territorial de $64,282 \mathrm{~km}^{2}$, equivale a $3.3 \%$ de la superficie del territorio nacional, y se divide políticamente en 76 municipios (http://mapserver.inegi.gob.mx/dsist/municipios/reporte.cfm).

En el estado se reconocen cuatro grandes regiones: las serranías del norte -derivadas del Eje Neovolcánico-, la cuenca del río Balsas, la zona de la costa y la zona de la montaña (Paucic, 1980). El gradiente altitudinal va de 0 a $3,500 \mathrm{~m}$ snm y las principales elevaciones son el cerro Teotepec con 3,500 m y los cerros Los Alzados y El Veladero, con 3,198 y 3,192 m, respectivamente (Paucic, 1980).

Los tipos climáticos van desde los cálidos semi-secos (BS) en las partes bajas de la cuenca del río Balsas, hasta los templados subhúmedos $(\mathrm{CW})$ en las partes altas de la Sierra Madre del Sur. El estado se caracteriza por tener una época de lluvias ubicada en la mitad calurosa del año (de mayo a octubre), alternando con un período extremadamente seco, en la mitad fría del año (de noviembre a abril) (Paucic, 1980).

Como resultado de las diversas condiciones climáticas,

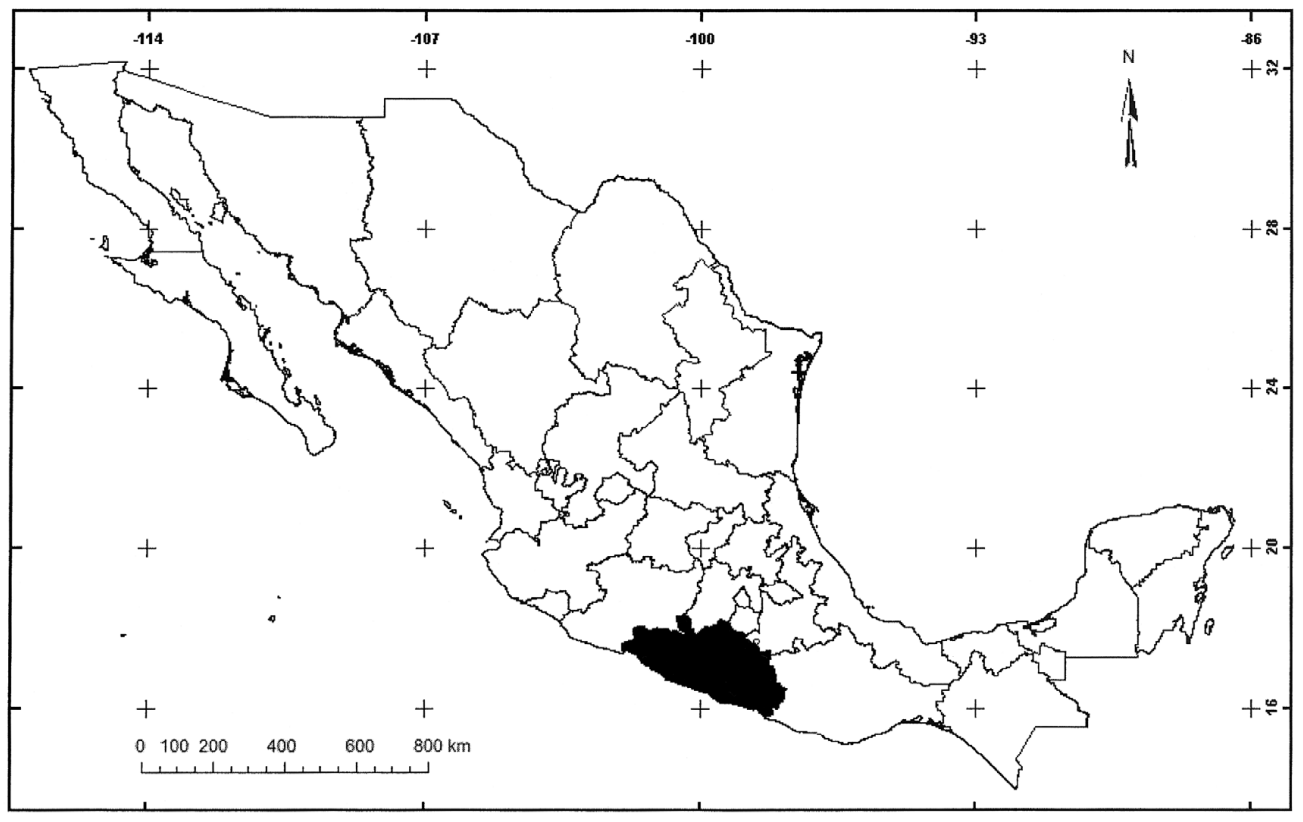

Figura 1. Ubicación del estado de Guerrero en la República Mexicana. 
edafológicas y topográficas, en el estado se presentan varios tipos de vegetación clasificados, de acuerdo con Rzedowski (1978), como: bosque de coníferas, bosque de Quercus, bosque de Pinus y Quercus, bosque mesófilo de montaña, bosque tropical caducifolio, bosque tropical subcaducifolio, bosque de galería, bosque de enebros o Juniperus, y palmar. Los bosques de coníferas, junto con los de Quercus y los de Pinus y Quercus, ocupan la mayor parte de los macizos montañosos del estado. El bosque mesófilo de montaña se distribuye en la región centro y sureste de la vertiente de barlovento de la Sierra Madre del Sur y en algunas cañadas de la Sierra de Taxco; los bosques tropical caducifolio y subcaducifolio tienen una amplia distribución en la depresión del Balsas, en la costa, y en la vertiente de barlovento de la Sierra Madre del Sur. Los bosques de galería ocupan las márgenes de los ríos y los palmares se presentan en manchones entremezclados con los bosques tropicales. Otros tipos de vegetación que ocupan áreas más restringidas son el tular, el manglar, el pastizal y la vegetación halófila.

\section{Métodos}

Desde junio de 1997 hasta principios de 2004 revisamos, cotejamos la determinación y capturamos en una base de datos toda la información de los especímenes de herbario procedentes del estado de Guerrero, así como de los ejemplares tipo depositados en las siguientes colecciones institucionales: BM, CHAPA, CHAP, CICY, CIIDIR CODAGEM, ENCB, F, FCME, HEID, HUH, IEB, LG, LL, M, MA, MEXU, MICH, MO, NY, P, SEL, TEX, UAMIZ, UC, US, WU, XAL y Z.

El estudio del material herborizado se complementó con registros obtenidos de la revisión cuidadosa de material bibliográfico diverso (McVaugh, 1951; Hinton y Rzedowski, 1972; Smith y Downs, 1974, 1977, 1979; BurtUtley y Utley, 1988; Ehlers, 1992; Till, 1992; VelázquezMontes y Domínguez-Licona, 2003). La base de datos así generada, que consta de 1,250 registros correspondientes a 797 colectas, se ha integrado al Catálogo Electrónico de Monocotiledóneas Mexicanas, en el Herbario Metropolitano (UAMIZ).

Con el fin de analizar la distribución de las especies, los datos referentes a la ubicación geográfica (geoposición) de los sitios de colecta se obtuvieron directamente de las etiquetas de los especímenes de herbario y en el caso de que no existieran, se utilizaron mapas topográficos escala 1:250,000 (INEGI, cartas E13-6-9, E14-4, E14-5, E14-710, E14-8 y E14-11) para ubicar las localidades y obtener las coordenadas de la forma más precisa posible. Con estos datos y utilizando el programa ArcView GIS 8 (ESRI, 2003), se cotejó la ubicación municipal y se elaboraron los mapas de distribución. Con el fin de tener un marco de referencia para estimar la frecuencia de aparición de las especies en el estado, se utilizó una rejilla de $10 \mathrm{~km}$ por lado, basada en la cuadrícula universal transversa de Mercator (UTM).

Una parte sustancial del análisis fue la revisión y la actualización de la determinación de los ejemplares estudiados, lo que aunado al análisis crítico de la bibliografía pertinente nos permitió definir la flora bromeliológica registrada hasta ahora para el estado. Con el propósito de proporcionar a los interesados una herramienta para reconocer las especies de Bromeliaceae que crecen en Guerrero, incluimos claves dicotómicas para la identificación de los géneros y de las especies, así como un catálogo completo de los mismos y de los ejemplares que los respaldan.

Con el fin de determinar la semejanza de la flora bromeliológica del estado con la de las floras de áreas vecinas, se hizo un análisis de la composición de las mismas. Para ello se seleccionaron los estados circunvecinos, y en dos casos, con el fin de compensar el tamaño de las áreas, se consideraron dos o más estados (Colima-Jalisco y Estado de México-Morelos) como una sola región.

Con los datos de distribución (presencia-ausencia) se construyó una matriz que se usó para obtener los índices de similitud de Jaccard y para llevar a cabo los análisis de agrupamiento. Este procedimiento permitió determinar, por un lado, las afinidades de la flora bromeliológica del estado con respecto a la de algunas áreas adyacentes, y por el otro, evaluar la semejanza de la flora misma entre los diferentes tipos de vegetación presentes en Guerrero. Para efectuar los análisis de agrupamiento se utilizó el método UPGMA (Unweighted pair-group arithmetic average clustering). El análisis se corrió con el programa NTSYSpc2.0 (Rohlf, 1998).

Con el propósito de tener una idea del estado que guarda el conocimiento y la exploración de la flora bromeliológica en el estado, generamos una curva de acumulación de especies en función del tiempo, utilizando la información de los años de recolección de las etiquetas de herbario y considerando como muestras al total de colecciones hechas cada 10 años, a partir de 1800, año en el que se tiene el primer registro de una Bromeliaceae para el estado. Además, generamos una curva de rarefacción (Colwell y Coddington, 1994) y los estimadores Chao2 y Jackknife2 (Chao, 1984; Colwell y Coddington, 1994). Para ello se contruyó una matriz de especies contra muestras, tomando en cuenta la abundancia de cada taxon. Los algoritmos se calcularon usando para ello el programa EstimateS Ver. 7 (Colwell, 2004).

\section{Resultados y discusión}

Como resultado de la revisión de 1,250 pliegos de herbario, correspondientes a 797 recolecciones diferentes, podemos decir que la flora bromeliológica hasta ahora conocida para Guerrero consta de 88 especies ubicadas en 11 géneros y 


\section{Valeria Angélica Pulido-EsparZa, Ana Rosa LóPez-Ferrari y Adolfo Espejo-Serna}

representa $25.73 \%$ de los 342 taxa de la familia registrados para el país (Espejo et al., 2004). Los géneros con mayor número de especies en la entidad son Tillandsia con $44 \mathrm{y}$ Pitcairnia con 14, en tanto que Billbergia, Racinaea, Ursulaea y Fosterella presentan un solo taxon. Se registran por primera vez para el estado los géneros Racinaea y Ursulaea, así como las siguientes especies: Catopsis berteroniana, Hechtia reflexa, Racinaea ghiesbreghtii, Tillandsia andrieuxii, T. gymnobotrya, T. magnusiana, T. marabascoensis, T. polystachia, T. rettigiana, T. tricolor y Ursulaea tuitensis.

De los nombres citados con anterioridad para el estado (Smith y Downs, 1974, 1977, 1979), excluimos 19 (ver catálogo), tres debido a que los consideramos como sinónimos, 12 por ser nombres mal aplicados debido a malas identificaciones, tres debido a actualizaciones nomenclaturales y uno más porque no hemos visto material que corresponda a dicho taxon.

Como ya lo habíamos apuntado anteriormente (Espejo y López-Ferrari, 1998), el estado de Guerrero es el que tiene mayor porcentaje de bromeliáceas endémicas, ya que 18 de las 88 especies presentes en él, es decir, $20.45 \%$ (una quinta parte), se encuentran exclusivamente dentro de sus límites (cuadro 1). Dichos taxa se ubican en tres géneros: Hechtia (4 especies), Pitcairnia (6) y Tillandsia (8). Asimismo, del total de los taxa que crecen en la entidad, 59 (67.04\%) son endémicos de México y se ubican en siete géneros, Bromelia (2 especies), Catopsis (1), Hechtia (7), Pitcairnia (12), Tillandsia (33), Ursulaea (1) y Viridantha (3).

Cabe destacar además que de las 18 especies endémicas de Guerrero, 9 (50\%) son, hasta donde sabemos, endemitas de un solo municipio (cuadro 1, figura 2) y también es notable que de las siete especies de Hechtia y de las 14 de Pitcairnia reportadas para el estado, cuatro $(57.1 \%)$ y seis (42.8\%), respectivamente, sean endémicas del mismo. Además, 13 taxa más son endémicos de Guerrero y de dos o tres estados vecinos, con poblaciones relativamente cercanas a los límites con Guerrero (cuadro 2), lo que reafirma la importancia del estado como centro de endemismo.

En lo tocante a su distribución conocida, podemos señalar que la mayoría de los registros se concentran en las partes centro-oriental y norte del estado y a lo largo de las diversas carreteras que lo recorren (figura 3). De los 76 municipios que conforman el estado, sólo para 54 (71.1\%) se tienen colecciones de Bromeliaceae (cuadro 3) y de siete de estos últimos sólo se conoce un registro. Para 27 (50.9\%) de los 53 municipios citados se tienen seis registros o menos que incluyen cinco especies o menos y en la mayoría de los casos, cada registro corresponde a una especie diferente. Es notable que de los 797 registros usados para el análisis (ver catálogo), $362(45.4 \%)$ se concentran en cuatro municipios, Chilpancingo, Eduardo Neri, José

Cuadro 1. Especies de Bromeliaceae endémicas del estado de Guerrero.

\begin{tabular}{|c|c|}
\hline Especie & Municipio \\
\hline Hechtia carlsoniae & Buenavista de Cuéllar, Taxco de Alarcón \\
\hline Hechtia melanocarpa & $\begin{array}{l}\text { Chilpancingo de los Bravo, Copalillo, Eduardo Neri, Huitzuco de los Figueroa, } \\
\text { Mártir de Cuilapan, Tepecoacuilco de Trujano }\end{array}$ \\
\hline Hechtia mooreana & $\begin{array}{l}\text { Chilapa de Álvarez, Copalillo, Eduardo Neri, Mártir de Cuilapan, Tepecoacuilco } \\
\text { de Trujano }\end{array}$ \\
\hline Hechtia pumila & Chilpancingo de los Bravo, Tepecoacuilco de Trujano \\
\hline Pitcairnia flavescentia & Chilpancingo de los Bravo \\
\hline Pitcairnia leprosa & Coahuayutla de José María Izazaga, Coyuca de Catalán, José Azueta \\
\hline Pitcarnia modesta & Chilpancingo de los Bravo \\
\hline Pitcairnia mooreana & Chilpancingo de los Bravo \\
\hline Pitcairnia sordida & $\begin{array}{l}\text { Coyuca de Catalán, Eduardo Neri, General Heliodoro Castillo, San Miguel } \\
\text { Totolapan }\end{array}$ \\
\hline Pitcairnia tillandsioides & San Miguel Totolapan \\
\hline Tillandsia guerreroensis & Juan R. Escudero, Tecpan de Galeana \\
\hline Tillandsia ilseana & Tixtla de Guerrero \\
\hline Tillandsia mirabilis & Atlixtac \\
\hline Tillandsia ortgiesiana & Acapulco de Juárez \\
\hline Tillandsia paraisoensis & Atoyac de Álvarez, Chilpancingo de los Bravo, General Heliodoro Castillo \\
\hline Tillandsia pentasticha & $\begin{array}{l}\text { Chilapa de Álvarez, Chilpancingo de los Bravo, Coyuca de Benítez, Leonardo } \\
\text { Bravo, Malinaltepec, Tlapa de Comonfort }\end{array}$ \\
\hline Tillandsia taxcoensis & Taxco de Alarcón \\
\hline Tillandsia trauneri & Coyuca de Catalán \\
\hline
\end{tabular}




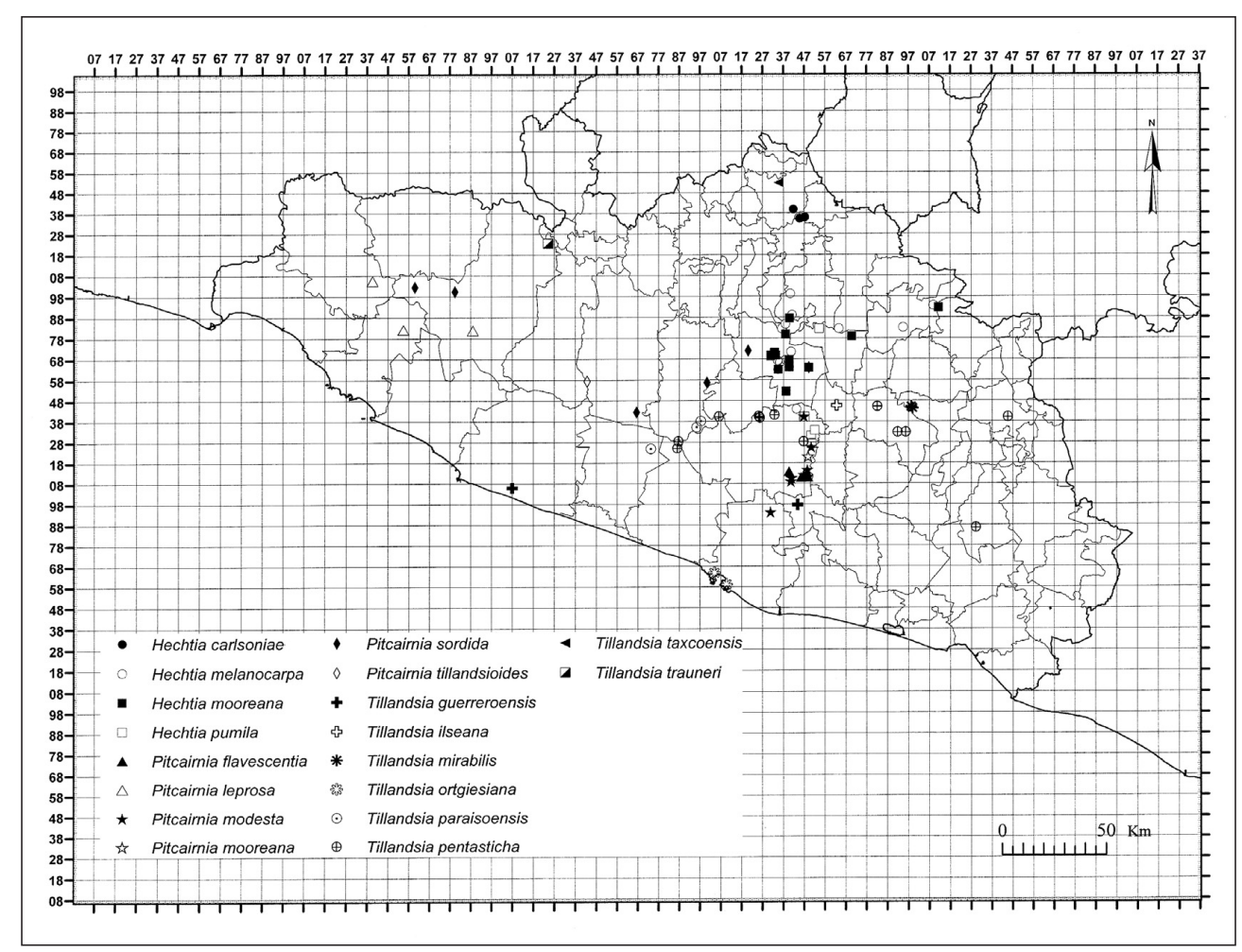

Figura 2. Especies de Bromeliaceae endémicas del estado de Guerrero.

Azueta y Leonardo Bravo. Además, casi $70 \%$ de los registros conocidos provienen de sólo 11 municipios (cuadro 3). Esto indica la gran heterogeneidad en la distribución de las recolecciones en la entidad y evidencia la preferencia que hasta el momento se presenta en la selección de los sitios de colecta.

En cuanto a la representación de las especies en las colecciones, existe también un claro sesgo hacia unos pocos taxa. De los 88 presentes en el estado, sólo 25 (28.4\%) cuentan con 10 registros o más (ver catálogo), en tanto que $14(15.9 \%)$ se encuentran representados únicamente por un espécimen.

En Flora Neotropica (Smith y Downs, 1974, 1977, 1979), único trabajo que hasta el momento aportaba información integral sobre las bromeliáceas de Guerrero, se citan para el estado 52 especies distribuidas en 6 géneros, basándose en 84 colecciones (registros). Como resultado de nuestro trabajo contamos ahora con 11 géneros, 88 especies y 797 colecciones. Los registros aumentaron un $89.46 \%$ (713) en el mismo lapso de tiempo. Descontando las sinonimias, las especies mal entendidas, los cambios nomenclaturales, etc., tenemos un incremento neto de 36 especies $(41 \%)$ en un promedio de 27 años (1977-2004), es decir, más de una especie añadida por año.
Cuadro 2. Especies endémicas de Guerrero y regiones adyacentes.

\begin{tabular}{ll}
\hline Especie & Distribución \\
\hline Bromelia hemisphaerica & $\begin{array}{l}\text { Guerrero, Estado de México y } \\
\text { Morelos }\end{array}$ \\
Hechtia caerulea & Guerrero y Estado de México \\
Hechtia reflexa & Guerrero y Michoacán \\
Hechtia sphaeroblasta & Guerrero, Oaxaca y Puebla \\
Pitcairnia hintoniana & Guerrero y Estado de México \\
Pitcairnia karwinskyana & Guerrero, Estado de México y \\
Pitcairnia pteropoda & Guerelos \\
Tillandsia circinnatioides & Guerrero, Morelos, Oaxaca y Puebla \\
Tillandsia kalmbacheri & Guerrero y Oaxaca \\
Tillandsia langlasseana & Guerrero, Estado de México y \\
Tillandsia laui & Michoacán \\
Tillandsia lydiae & Guerrero y Oaxaca \\
Tillandsia oaxacana & Guerrero y Morelos \\
Tillandsa supermexicana & Guerrero y Oaxaca \\
\hline & Guerrero y Oaxaca
\end{tabular}




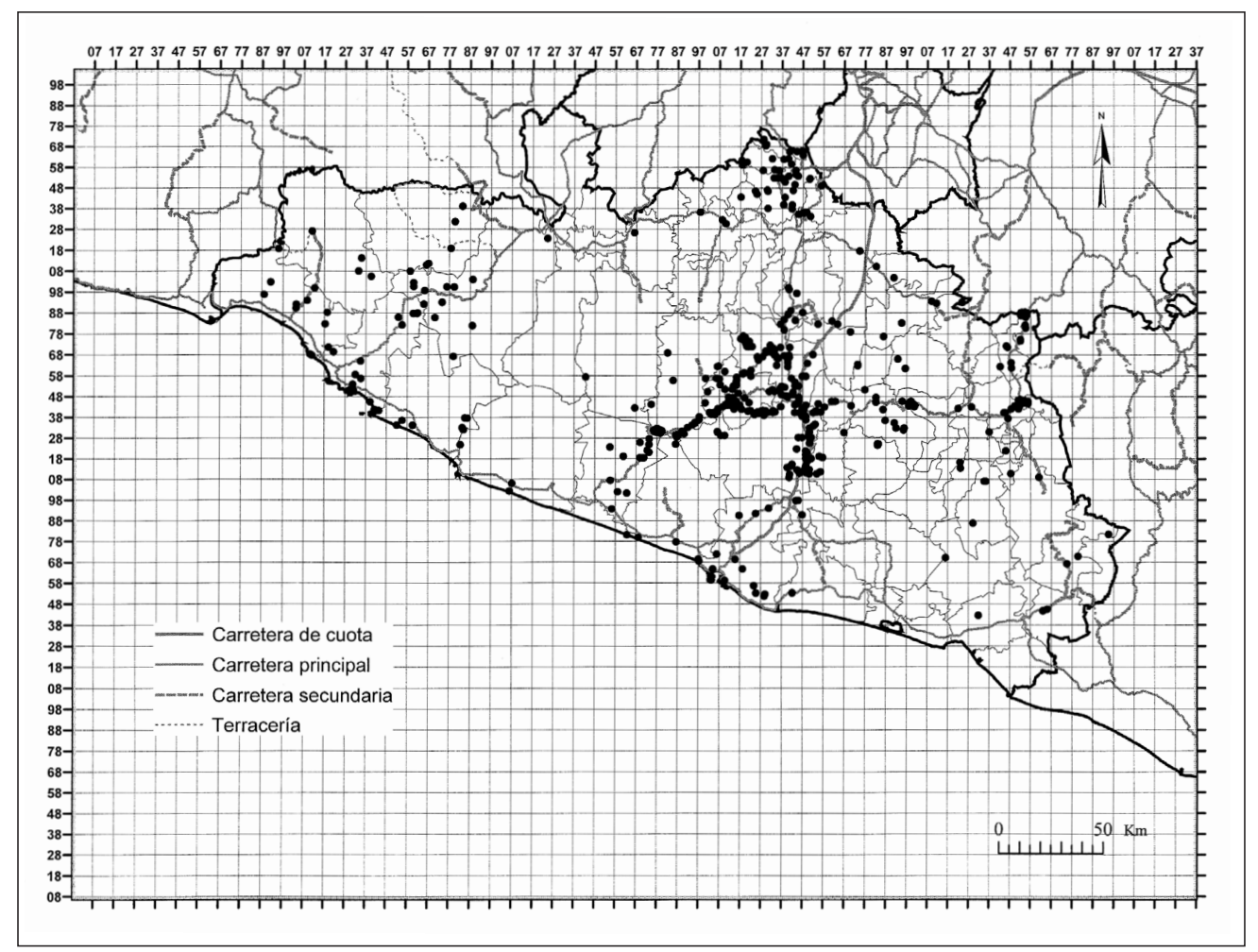

Figura 3. Total de colecciones $(\bullet)$ de Bromeliaceae registradas para Guerrero.

Con el propósito de tener una representación más clara de estos datos, elaboramos una curva de acumulación de especies-área (figura $4, \diamond$ ). Dicha curva se encuentra lejos de llegar a una asíntota, lo cual en principio nos indica que falta aún trabajo para contar con un conocimiento adecuado de la familia en el estado. Cabe mencionar, sin embargo, que la curva está basada en muestras heterogéneas, azarosas y sin ningún patrón temporal y/o espacial, debido a la condición misma de las recolecciones. Con el fin de tener una estimación estadísticamente más confiable, construimos una curva de rarefacción (ם) y la comparamos con las curvas obtenidas utilizando los algoritmos de Chao2 (A) y Jackknife2 (メ) para estimar la riqueza (Colwell y Coddington, 1994). Como resultado de este último análisis, tenemos que el número de especies aquí reportado (88) se encuentra por debajo del obtenido por dichos algoritmos, que alcanzan las cifras de 99 (Chao2) y 115 (Jackknife2), es decir, que deberíamos tener entre 10 y 27 especies más de las que aquí reportamos, considerando el número de colecciones con el cual contamos actualmente.

Otro dato interesante a considerar es que de las 36 especies que reportamos aquí por primera vez para el estado, desde el trabajo de Smith y Downs (1974, 1977, 1979), $14(38.8 \%)$ son taxa que se han descrito como nuevos para la ciencia con material tipo procedente del estado de
Cuadro 3. Número de especies y de colecciones por municipio.

\begin{tabular}{lcc}
\hline Municipio & No. especies & $\begin{array}{c}\text { No. registros } \\
\text { (colecciones) }\end{array}$ \\
\hline Acapulco de Juárez & 9 & 28 \\
Acatepec & 0 & 0 \\
Ahuacuotzingo & 3 & 4 \\
Ajuchitlán del Progreso & 0 & 0 \\
Alcozauca de Guerrero & 0 & 0 \\
Alpoyeca & 5 & 9 \\
Apaxtla & 0 & 0 \\
Arcelia & 1 & 1 \\
Atenango del Río & 2 & 2 \\
Atlamajalcingo del Monte & 1 & 1 \\
Atlixtac & 9 & 19 \\
Atoyac de Álvarez & 13 & 25 \\
Ayutla de los Libres & 0 & 0 \\
Azoyú & 1 & 1 \\
Benito Juárez & 2 & 2 \\
Buenavista de Cuéllar & 5 & 11 \\
Chilapa de Álvarez & 9 & 12 \\
Chilpancingo de los Bravo & 45 & 155 \\
Coahuayutla de José María Izazaga & 5 & 7 \\
Cocula & 0 & 0 \\
Copala & 0 & 0 \\
Copalillo & 3 & 5 \\
Copanatoyac & 0 & 0 \\
Coyuca de Benítez & 4 & 5 \\
Coyuca de Catalán & 14 & 26 \\
\hline & & \\
\hline & &
\end{tabular}


Flora BromeliolóGicA DE GUERRERO

Cuadro 3. Continuación

\begin{tabular}{|c|c|c|}
\hline Municipio & No. especies & $\begin{array}{l}\text { No. registros } \\
\text { (colecciones) }\end{array}$ \\
\hline Cuajinicuilapa & 0 & 0 \\
\hline Cualác & 0 & 0 \\
\hline Cuautepec & 0 & 0 \\
\hline Cuetzala del Progreso & 0 & 0 \\
\hline Cutzamala de Pinzón & 0 & 0 \\
\hline Eduardo Neri & 19 & 104 \\
\hline Florencio Villarreal & 0 & 0 \\
\hline General Canuto A. Neri & 0 & 0 \\
\hline General Heliodoro Castillo & 17 & 40 \\
\hline Huamuxtitlán & 7 & 12 \\
\hline Huitzuco de los Figueroa & 4 & 4 \\
\hline Iguala de la Independencia & 0 & 0 \\
\hline Igualapa & 0 & 0 \\
\hline Ixcateopan de Cuauhtémoc & 2 & 2 \\
\hline José Azueta & 20 & 48 \\
\hline Juan R. Escudero & 7 & 10 \\
\hline La Unión de Isidoro Montes de Oca & 12 & 12 \\
\hline Leonardo Bravo & 20 & 55 \\
\hline Malinaltepec & 6 & 7 \\
\hline Mártir de Cuilapan & 5 & 7 \\
\hline Metlatónoc & 1 & 1 \\
\hline Mochitlán & 5 & 5 \\
\hline Olinalá & 1 & 1 \\
\hline Ometepec & 2 & 2 \\
\hline Pedro Ascencio Alquisiras & 1 & 1 \\
\hline Petatlán & 7 & 11 \\
\hline Pilcaya & 7 & 14 \\
\hline Pungarabato & 0 & 0 \\
\hline Quechultenango & 3 & 3 \\
\hline San Luis Acatlán & 3 & 3 \\
\hline San Marcos & 1 & 1 \\
\hline San Miguel Totolapan & 2 & 2 \\
\hline Taxco de Alarcón & 13 & 27 \\
\hline Tecoanapa & 0 & 0 \\
\hline Tecpan de Galeana & 2 & 2 \\
\hline Teloloapan & 3 & 3 \\
\hline Tepecoacuilco de Trujano & 6 & 9 \\
\hline Tetipac & 8 & 8 \\
\hline Tixtla de Guerrero & 14 & 26 \\
\hline Tlacoapa & 3 & 6 \\
\hline Tlacochistlahuaca & 2 & 3 \\
\hline Tlalchapa & 0 & 0 \\
\hline Tlalixtaquilla de Maldonado & 4 & 5 \\
\hline Tlapa de Comonfort & 7 & 15 \\
\hline Tlapehuala & 0 & 0 \\
\hline Xalpatláhuac & 3 & 3 \\
\hline Xochihuehuetlán & 8 & 12 \\
\hline Xochistlahuaca & 0 & 0 \\
\hline Zapotitlán Tablas & 2 & 2 \\
\hline Zirándaro & 4 & 5 \\
\hline Zitlala & 6 & 9 \\
\hline
\end{tabular}

Guerrero. También es importante notar que algunas especies como T. nuyooensis, T. setiformis y T. nolleriana, entre otras, se conocen de estados vecinos en regiones cercanas a las fronteras con Guerrero, por lo que en un futuro podrían ser encontradas en la entidad.

Por otra parte y como resultado de la comparación de la flora bromeliológica de Guerrero con las de las regiones vecinas, se observa que el estado de Oaxaca ocupa el
Cuadro 4. Número total de especies y endémicas por estado y/o región.

\begin{tabular}{lccc}
\hline Estado & $\begin{array}{c}\text { Total de } \\
\text { especies }\end{array}$ & $\begin{array}{c}\text { Especies } \\
\text { endémicas }\end{array}$ & $\begin{array}{c}\text { Porcentaje } \\
\text { de endemismo }\end{array}$ \\
\hline Chiapas & 126 & 18 & 14.28 \\
Oaxaca & 139 & 28 & 20.14 \\
Michoacán & 47 & 3 & 6.30 \\
Colima-Jalisco & 76 & 10 & 13.10 \\
México-Morelos-Puebla & 84 & 7 & 8.30 \\
Guerrero & 88 & 18 & 20.45 \\
\hline
\end{tabular}

primer lugar tanto por el número total de especies como por la cifra de taxa endémicos; sin embargo, el porcentaje más alto de especies endémicas lo presenta Guerrero (cuadro 4). Es importante hacer notar la aparente pobreza de especies, y por ende de endemitas, que presenta Michoacán.

El área que presenta una mayor afinidad florística con Guerrero es el conjunto Jalisco-Colima, en tanto que la que presenta una mayor disimilitud es la de Chiapas.

En el dendrograma de la figura 5 es posible distinguir dos grandes grupos $(\mathrm{J}=0.30)$, uno constituido por Chiapas $\mathrm{y}$ Oaxaca y otro formado por las demás regiones comparadas. Esto indica que las floras presentes en Chiapas y Oaxaca son muy distintas de las del resto, lo que probablemente se deba al elevado número de elementos mesoamericanos presentes en ellas ( $c f$. Rzedowski 1978:74-75). Como puede también advertirse, las regiones Michoacán y JaliscoColima son prácticamente iguales entre sí, y junto con el área Estado de México-Morelos, comparten con Guerrero una buena proporción de especies. Esto sugiere que Guerrero tiene una doble influencia en su composición florística, por una parte la de la vertiente pacífica del país, dentro de la provincia fisiográfica Sierra Madre del Sur, y por otra la del Eje Volcánico Transversal.

Al aplicar un análisis similar considerando los tipos de vegetación presentes en el estado (sensu Rzedowski, 1978), se obtuvo el dendrograma de la figura 6 , en el cual es posible distinguir cuatro conjuntos $(\mathrm{J}=0.22)$ agrupados a su vez en dos grandes grupos $(\mathrm{J}=0.12)$, uno en donde encontramos los tipos de vegetación con afinidades templadas (bosque de Quercus [BQ], bosque mesófilo de montaña [BM], bosque de Quercus-Pinus [BQP] y bosque de coníferas [BC]), y otro en el cual se distinguen comunidades con afinidades más cálidas (bosque tropical caducifolio [BTC] y bosque tropical subcaducifolio [BTS]), así como vegetación de transición o de condiciones ambientales muy específicas (bosques de galería [BG] y de Juniperus $[\mathrm{BJ}])$. 


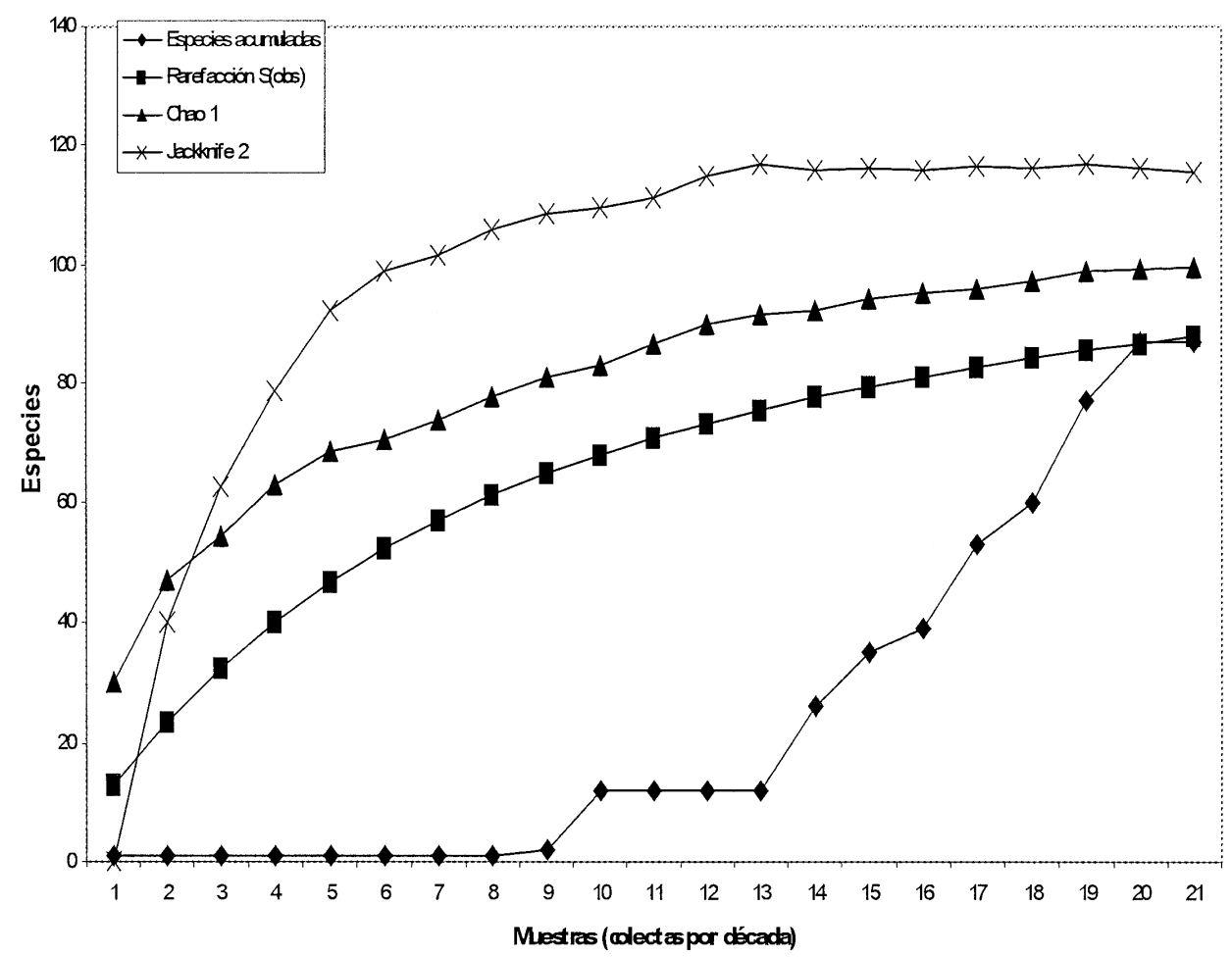

Figura 4. Curvas de acumulación de especies y de rarefacción.

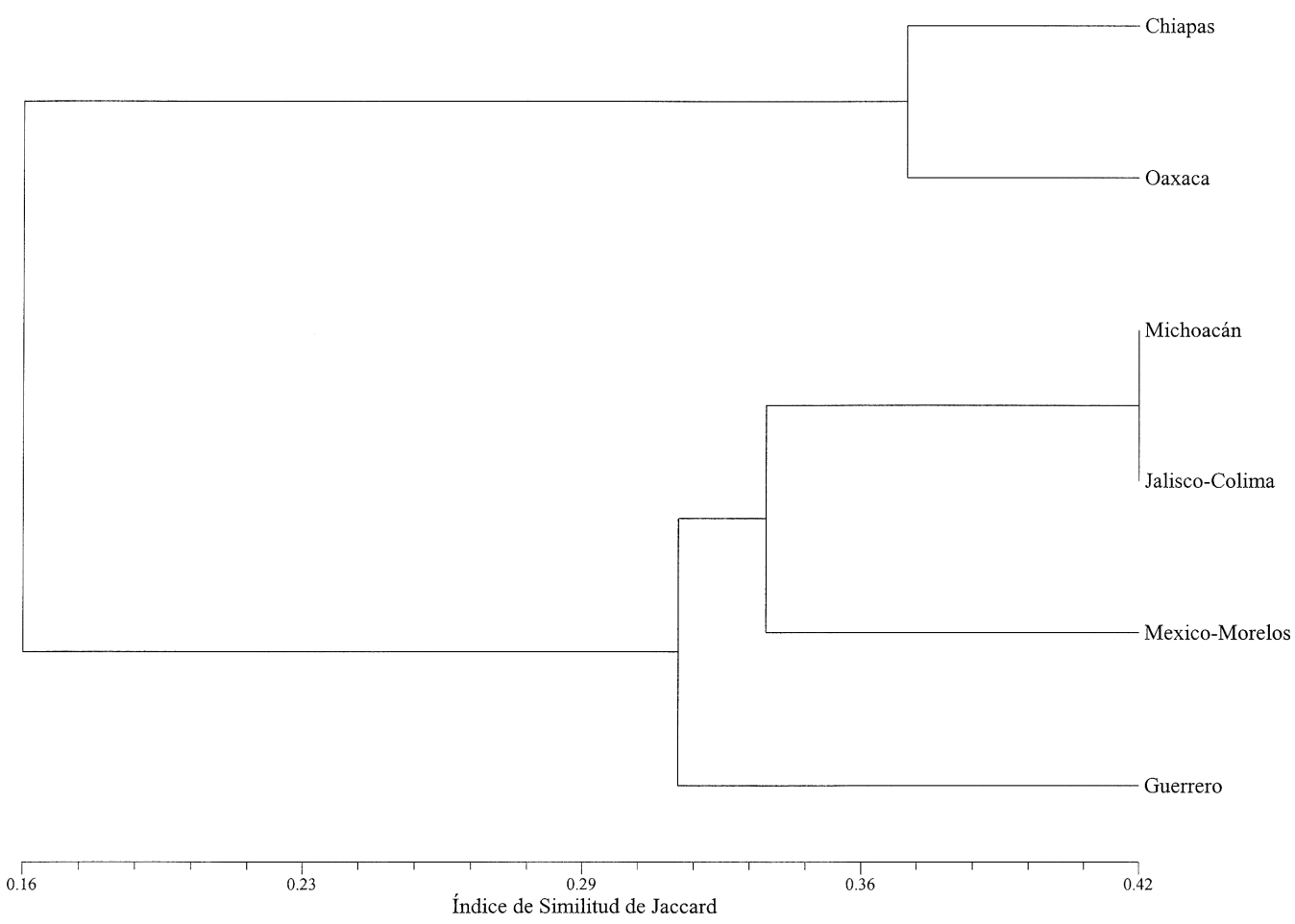

Figura 5. Similitud de la flora bromeliológica entre las áreas comparadas. 


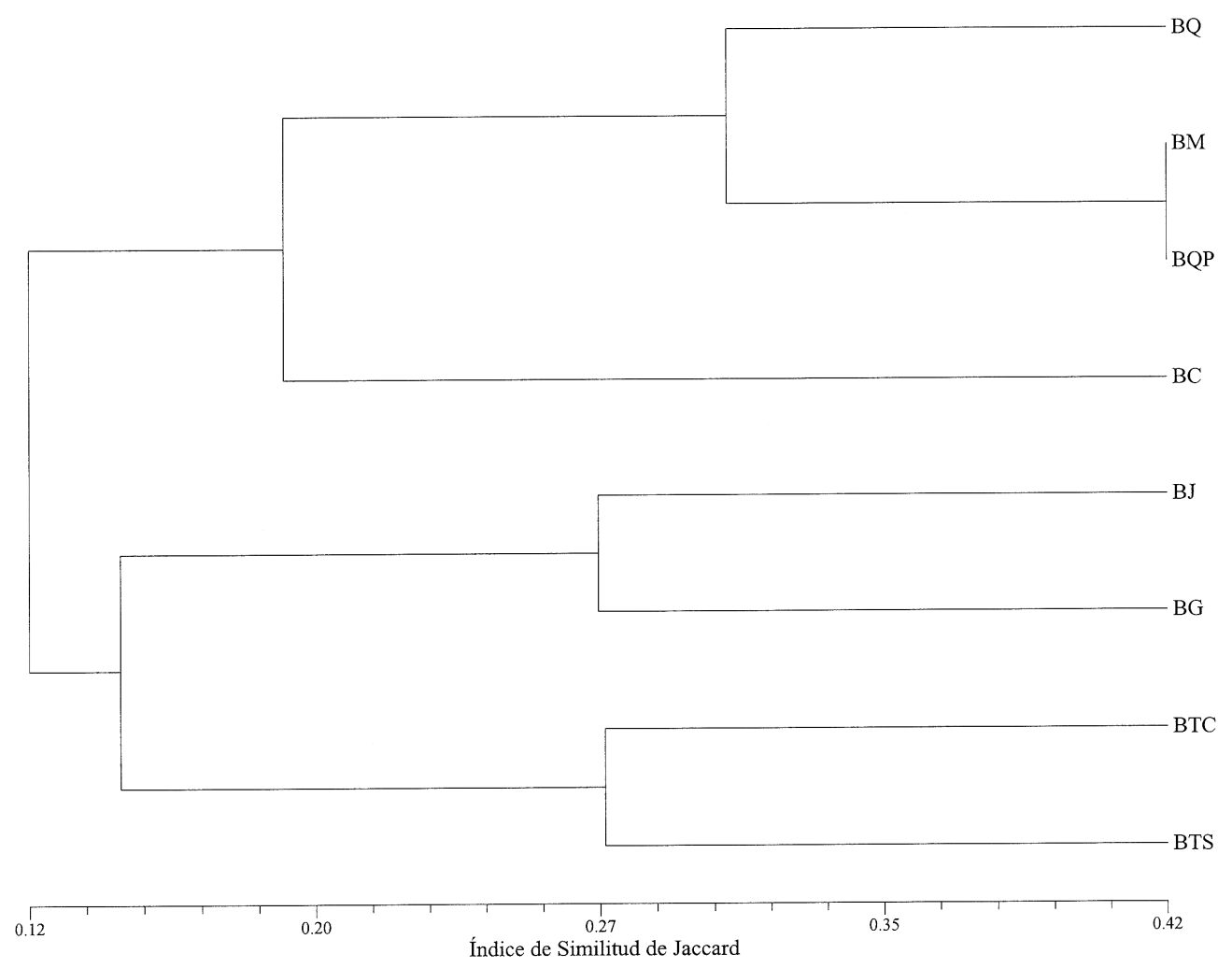

Figura 6. Similitud de la flora bromeliológica entre los tipos de vegetación (sensu Rzedowski, 1978) presentes en el estado. BQ: bosque de Quercus; BM: bosque mesófilo de montaña; BQP: bosque de Quercus-Pinus; BJ: bosque de enebros o Juniperus; BG: bosque de galería; BTC: bosque tropical caducifolio; BTS: bosque tropical subcaducifolio.

El primer lugar en riqueza de especies lo tiene el bosque de Quercus con 42 taxa, seguido por los bosques tropical caducifolio con 33 y tropical subcaducifolio con 27. El tipo de vegetación con menor número de especies es el bosque de Juniperus, en donde crecen sólo cuatro taxa.

Hechtia pumila, Pitcairnia oaxacana, P. pteroda, P. tillandsioides, Racinaea ghiesbreghtii, Tillandsia andrieuxii, T. calothyrsus, T. ilseana, T. kalmbacheri, T. magnusiana, T. polystachia, T. trauneri, Ursulaea tuitensis $\mathrm{y}$ Viridantha ignesiae crecen de manera exclusiva en bosques de Quercus. Los taxa registrados sólo para el bosque tropical caducifolio son Billbergia pallidiflora, Bromelia hemisphaerica, Hechtia caerulea, H. carlsoniae, H. melanocarpa, H. mooreana, Pitcairnia hintoniana, Tillandsia circinnatioides, T. concolor, T. lydiae, $T$. mirabilis y $T$. pseudobaileyi.

Diez especies crecen únicamente en el bosque tropical subcaducifolio: Bromelia karatas, B. palmeri, Fosterella micrantha, Hechtia reflexa, Tillandsia balbisiana, T. pseudosetacea, T. fuchsii, T. guerreroensis, T. marabascoensis y T. xerographica.

Aunque escasas, existen algunas especies de amplia distribución que se pueden encontrar en diversos tipos de vegetación: Tillandsia achyrostachys, T. bourgaei, $T$. caput-medusae, T. hintoniana, T. ionantha, T. makoyana, T. fasciculata, T. quaquaflorifera, T. recurvata, T. schiedeana y Viridantha plumosa.

En el apéndice 1 presentamos el catálogo florístico y los mapas de distribución de los taxa de bromeliáceas presentes en Guerrero. Se presentan primero los datos obtenidos de la revisión de los ejemplares de herbario, indicando al final los acrónimos respectivos según Holmgren $e t$ al., (1990). El asterisco que acompaña algunos acrónimos indica que los ejemplares se encontraban en proceso y aún no se integraban a las colecciones respectivas. Posteriormente se enlistan las localidades obtenidas de diversas fuentes bibliográficas, indicando entre paréntesis las mismas.

Con el fin de facilitar a los lectores la ubicación de los taxa, también proporcionamos claves de identificación a nivel génerico y a nivel específico.

A manera de conclusión podemos decir que el inventario de las Bromeliaceae de Guerrero dista de estar completo y que hay una urgente necesidad de aumentar la exploración de la familia en el estado, pero de acuerdo con planes de recolección dirigidos a las áreas poco conocidas y alejadas de las carreteras. 


\section{Valeria Angélica Pulido-Esparza, Ana Rosa LópeZ-Ferrari y Adolfo EsPejo-Serna}

\section{Agradecimientos}

Queremos agradecer a Ivón Ramírez, Alejandro Zavala, Jorge Meave, Bruce Holst, Jacqueline Ceja y un árbitro anónimo por la revisión crítica del manuscrito, el cual mejoró notablemente con sus comentarios y sugerencias. Los curadores de los herbarios mencionados nos facilitaron la consulta del material. Deseamos expresar nuestra gratitud al personal del Laboratorio de Plantas Vasculares y del Herbario FCME, de la Facultad de Ciencias (UNAM), por habernos permitido el acceso a todo el material de Bromeliaceae, incluyendo los ejemplares en proceso, lo que nos facilitó enormemente el trabajo y nos permtió enriquecer y completar los datos para el análisis aquí presentado. Parte de este trabajo pudo llevarse a cabo gracias al apoyo de la beca Elizabeth Bascom otorgada en el año 2004 por el Missouri Botanical Garden a A.R. LópezFerrari.

\section{Literatura citada}

Aguirre-León E. 1992. Vascular epiphytes of Mexico: a preliminary inventory. Selbyana 13:72-76.

Benzing D.H. 2000. Bromeliaceae: Profile of an Adaptative Radiation. Cambridge University Press, Cambridge.

Brücher H. 1989. Useful Plants of Neotropical Origin and their Wild Relatives. Springer-Verlag, Nueva York.

Burt-Utley K. y Utley J.F. 1988. New and noteworthy species of Hechtia (Bromeliaceae) from Guerrero, Mexico. Systematic Botany 13:276-282.

Chao A. 1984. Nonparametric estimation of the number of classes in a population. Scandinavian Journal of Statistics 11:265270

Clark T.F. 1965. Plant fibers in the paper industry. Economic Botany 19:394-405.

Colwell R.K y Coddington J.A. 1994. Estimating terrestrial biodiversity through extrapolation. Philosophical Transactions of the Royal Society (Series B) 345:101-188.

Colwell R.K. 2004. Estimates: statistical estimation of species richness and shared species from samples. Version 7. User's Guide and Application.

$<$ http://viceroy.eeb.uconn.edu/estimates >

Diego N. y Lozada L. 1994. Estudios Florísticos en Guerrero No. 3. Laguna de Tres Palos. Las Prensas de Ciencias, Facultad de Ciencias, Universidad Nacional Autónoma de México, México, D.F.

Diego-Pérez N. 2000. Estudios Florísticos en Guerrero No. 10. Lagunas Playa Blanca, El Potosí, Salinas del Cuajo y Zonas Circundantes. Las Prensas de Ciencias, Facultad de Ciencias, Universidad Nacional Autónoma de México, México, D.F.

Diego-Pérez N., Peralta-Gómez S. y Ludlow-Wiechers B. 2001. Estudios Florísticos en Guerrero No. 11. El Jilguero, Bosque Mesófilo de Montaña. Las Prensas de Ciencias, Facultad de Ciencias, Universidad Nacional Autónoma de México, México, D.F.

Ehlers R. 1992. Tillandsia laui, an additional collection. Journal of the Bromeliad Society 42:74.

Espejo A. y López-Ferrari A.R. 1998. Current floristic and phyto- geographic knowledege of Mexican Bromeliaceae. Revista de Biología Tropical 46:493-513.

Espejo A. y López-Ferrari A.R. 2003. Las Monocotiledóneas (Liliopsida) mexicanas con potencial ornamental. En: MejíaMuñoz J.M. y Espinosa-Flores A. Comps. Plantas Nativas de México con Potencial Ornamental, pp. 20-26, Universidad Autónoma Chapingo, Texcoco.

Espejo A., López-Ferrari A.R., Ramírez-Morillo I., Holst B.K., Luther H.E. y Till W. 2004. Checklist of Mexican Bromeliaceae with notes on species distribution by state and municipality, and levels of endemism. Selbyana 25:33-86.

ESRI. 2003. ArcView Ver. 8.1.

Fonseca R.M. y Lozada L. 1993. Estudios Florísticos en Guerrero No. 1. Laguna de Coyuca. Las Prensas de Ciencias, Facultad de Ciencias, Universidad Nacional Autónoma de México, México, D.F.

Fonseca R.M., Velázquez-Montes E. y Domínguez-Licona E. 2001. Estudios Florísticos en Guerrero No. 12. Carrizal de Bravos, Bosque Mesófilo de Montaña. Las Prensas de Ciencias, Facultad de Ciencias, Universidad Nacional Autónoma de México, México, D.F.

Gallardo C. 1996. Estudios Florísticos en Guerrero No. 8. Parque Ecológico La Vainilla, Zihuatanejo, Guerrero. Las Prensas de Ciencias, Facultad de Ciencias, Universidad Nacional Autónoma de México, México, D.F.

Gardner S. 1982. Tillandsias at Christmas in Mexico. Journal of the Bromeliad Society 32:261-262.

Gual M. 1995. Estudios Florísticos en Guerrero No. 6. El Cañón del Zopilote (Área Venta Vieja). Las Prensas de Ciencias, Facultad de Ciencias, Universidad Nacional Autónoma de México, México, D.F.

Guess V. y Guess R. 2001. Edible fruits of Bromelia plumieri and Bromelia pinguin from Chiapas. Journal of Bromeliad Society 51:51-55.

Hinton J. y Rzedowski J. 1972. George B. Hinton, collector of plants in southwestern Mexico. Journal of the Arnold Arboretum 53:141-181.

Holmgren P.K., Holmgren N.H. y Barnett L.C. Eds. 1990. Index Herbariorum. Part I: The Herbaria of the World. Regnum Vegetabile V. 120 ed. 8.

INEGI. 2003. Catálogo de Municipios e Integración Territorial. $<$ http://www.inegi.gob.mx/geo/default.asp?c=342>

Kirby R.H. 1963. Vegetable Fibers. Interscience Publisher, Londres.

Lozada L. 1994. Estudios Florísticos en Guerrero No. 2. Laguna de Mitla. Las Prensas de Ciencias, Facultad de Ciencias, Universidad Nacional Autónoma de México, México, D.F.

Lozada L., León M.E., Rojas J. y de Santiago R. 2003. Estudios Florísticos en Guerrero No. 13. Bosque Mesófilo de Montaña en El Molote. Las Prensas de Ciencias, Facultad de Ciencias, Universidad Nacional Autónoma de México, México, D.F.

Martínez M. 1990. Las Plantas Medicinales de México. Ed. Botas, México, D.F.

McVaugh R. 1951. The travels and botanical collections of Eugène Langlassé in Mexico and Colombia, 1898-1899. Candollea 13:167-211.

McVaugh R. 1989. Bromeliaceae. En: Anderson W.R. Ed. Flora Novo-Galiciana Vol. 15, pp. 4-79. The University of Michigan Herbarium, Ann Arbor.

Paucic A.W. 1980. Geografía General del Estado de Guerrero. 
FONAPAS, Gobierno del Estado, Chilpancingo.

Peralta-Gómez S. 1995. Estudios Florísticos en Guerrero No. 5. El Cañón del Zopilote (Área Papalotepec). Las Prensas de Ciencias, Facultad de Ciencias, Universidad Nacional Autónoma de México, México, D.F.

Pierce S. 2000. The use of Tillandsia species in ritual adornment in Qoosqo, Peru. Journal of Bromeliad Society 50:195-201.

Ríos R. y Khan B. 1998. List of ethnobotanical uses of Bromeliaceae. Journal of Bromeliad Society 48:75-87.

Rohlf F.J. 1998. NTSYSpc. Numerical Taxonomy and Multivariate Analysis System. Version 2.0. User Guide. University of New York. Stony Brook, Nueva York.

Rzedowski J. 1978. Vegetación de México. Limusa, México, D.F. Smith L.B. y Downs R.J. 1974. Pitcairnioideae (Bromeliaceae). Flora Neotropica 14:1-658.

Smith L.B. y Downs R.J. 1977. Tillandsioideae (Bromeliaceae). Flora Neotropica 14:659-1492.

Smith L.B. y Downs R.J. 1979. Bromelioideae (Bromeliaceae). Flora Neotropica 14:1493-2142.

Till W. 1992. A well-known new species: Tillandsia fuchsii. Journal of the Bromeliad Society 42:99-102.

Utley J.F. y Burt-Utley K. 1994. Bromeliaceae. En: Davidse G., Sousa M. y Carter A.O. Eds. Flora Mesoamericana Vol. 6,
Alismataceae a Cyperaceae, pp. 89-156. Universidad Nacional Autónoma de México, Missouri Botanical Garden y The Natural History Museum (London), México, D.F.

Vargas A. y Pérez A. 1996. Estudios Florísticos en Guerrero No. 7. Cerro Chiletépetl y Alrededores (Cuenca del Balsas). Las Prensas de Ciencias, Facultad de Ciencias, Universidad Nacional Autónoma de México, México, D.F.

Velázquez-Montes E. y Domínguez-Licona E. 2003. Estudios Florísticos en Guerrero No. 15. Cerro Teotepec. Las Prensas de Ciencias, Facultad de Ciencias, Universidad Nacional Autónoma de México, México, D.F.

Verduzco C. y Rodríguez L.C. 1995. Estudios Florísticos en Guerrero No. 4. El Rincón de la Vía. Las Prensas de Ciencias, Facultad de Ciencias, Universidad Nacional Autónoma de México, México, D.F.

Victoria A. 2001. Bromeliaceae. En: Rzedowski J. y Calderón de Rzedowski G. Eds. Flora Fanerogámica del Valle de México, pp. 1179-1187, Instituto de Ecología, A.C., Centro Regional del Bajío y Comisión Nacional para el Conocimiento y Uso de la Biodiversidad, Pátzcuaro.

Wolf J.H.D. y Flamenco A. 2003. Patterns in species richness and distribution of vascular epiphytes in Chiapas, Mexico. Journal of Biogeography 30:1689-1707.

Fecha de recepción: 19 de mayo de 2004

Versión corregida: 1 de octubre de 2004

Aceptado: 1 de octubre de 2004 


\section{Valeria Angélica Pulido-Esparza, Ana Rosa LóPeZ-Ferrari y Adolfo EsPejo-Serna}

Apéndice 1. Catálogo de las especies de Bromeliaceae presentes en Guerrero y claves para su identificación.

1. Hojas con los márgenes enteros, lisos.

2. Flores unisexuales; plantas dioicas Catopsis

2. Flores bisexuales; plantas hermafroditas.

3. Plantas terrestres a rupícolas; apéndices de las semillas apicales.

4. Ovario totalmente súpero; el verticilo floral petaloide actinomorfo; semillas bicaudadas Fosterella

4. Ovario parcial o totalmente ínfero, o si súpero entonces el verticilo petaloide zigomorfo; semillas unicaudadas....

Pitcairnia

3. Plantas epífitas o raramente terrestres, pero entonces los apéndices de la semillas total o principalmente basales, rectos en la madurez.

5. Sépalos asimétricos en contorno general, más anchos en su mitad apical

Racinaea

5. Sépalos simétricos en contorno general, más anchos en su mitad basal.

6. Estambres exertos, si inclusos entonces los pétalos de color violeta o verde claro; flores erectas..... Tillandsia

6. Estambres inclusos; pétalos de color verde oscuro; flores extendidas a nutantes.

Viridantha

1. Hojas con los márgenes espinosos a serrados.

7. Flores unisexuales; plantas dioicas.....

Hechtia

7. Flores bisexuales; plantas hermafroditas.

8. Flores de menos de $15 \mathrm{~mm}$ de largo; pétalos amarillos o raramente rosados a purpúreos Aechmea

8. Flores de más de $25 \mathrm{~mm}$ de largo; pétalos rojos, púrpuras, verdes o raramente blancos.

9. Filamentos libres.

10. Pétalos verdes; plantas epífitas; hojas variegadas Billbergia

10. Pétalos rojos, púrpura-obscuros o blancos; plantas terrestres o rupícolas.

11. Estambres más cortos que los pétalos; fruto una cápsula fusiforme.

Pitcairnia

11. Estambres más largos que los pétalos; fruto una baya globosa. Ursulaea

9. Filamentos connatos formando un tubo al cual se unen los pétalos.

Bromelia

\section{Aechmea Ruiz et Pav.}

1. Inflorescencias paniculadas, irregulares en contorno general; flores dísticas; brácteas del escapo rojas; pétalos amarillos.

A. bracteata

1. Inflorescencias tirsiformes, cilíndricas a cónicas en contorno general; flores polísticas; brácteas del escapo rosadas; pétalos rosados a purpúreos.

A. mexicana

1. Aechmea bracteata (Sw.) Griseb., Fl.Brit.W.I. 592. 1864.

Figura 7 DISTRIBUCIÓN: México a Colombia.

ALTITUD: 0-1100 m.

EJEMPLARES EXAMINADOS: Atoyac de Álvarez: $3.5 \mathrm{~km}$ al N de El Cacao, camino hacia El Fortín, $17^{\circ} 21^{\prime} 20^{\prime \prime} \mathrm{N}$, 100¹8'30" O, 1100 m, G. Campos y V. Valverde 1782 (FCME); a 1 km del ejido El Quemado por el arroyo grande, $17^{\circ} 11^{\prime} 40^{\prime \prime} \mathrm{N}, 100^{\circ} 17^{\prime} 30^{\prime \prime} \mathrm{O}, 600 \mathrm{~m}$, M. P. Ochoa s. n. (FCME*); camino a Las Selvas, J. Ramírez 147 (FCME).

Chilpancingo de los Bravo: El Rincón, $45 \mathrm{~km}$ adelante de Chilpancingo camino antiguo a Acapulco, 17 $17^{\prime} 40^{\prime \prime} \mathrm{N}$,

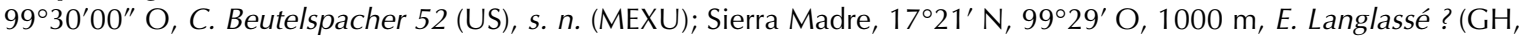

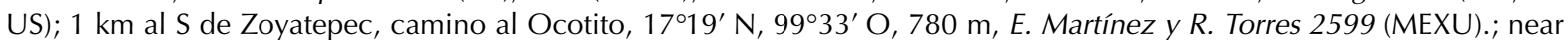
km 339 on hwy to Acapulco, below Acahuizotla, $17^{\circ} 22^{\prime} \mathrm{N}, 99^{\circ} 27^{\prime} \mathrm{W}$, ca. $940 \mathrm{~m}, \mathrm{H}$. E. Moore y A. Valiente 6205 
FLORA BROMELIOLÓGICA DE GUERRERO

(MEXU, UC, US); río La Hamaca, al SE de Soyatepec, 17¹9’30" N, 99³2'44" O, 800 m, L. C. Rodríguez M. 353L (FCME). Coahuayutla de José María Izazaga: $2.82 \mathrm{~km}$ al NE de San Cristóbal, $18^{\circ} 8$ '51' N, 101³1'4' O, $430 \mathrm{~m}$, J. Calónico13673 (FCME); in Vanilla Vinery, Coahuayula, 18¹9' N, 101 $44^{\prime}$ O, G. M. Emrick 49 (F); hacienda Coahuayutla

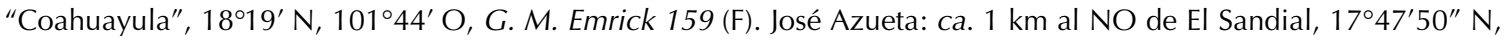

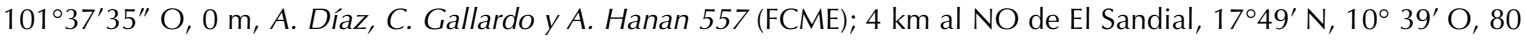

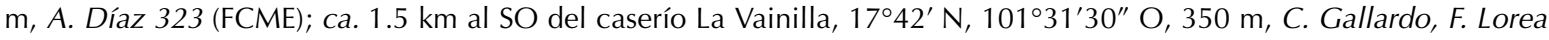
y A. Hanan 414 (FCME, MEXU); I. R. F. Vallecitos, $17^{\circ} 55^{\prime} \mathrm{N}, 101^{\circ} 19^{\prime} \mathrm{O}, 520 \mathrm{~m}$, G. B. Hinton et al. $10224(\mathrm{GH}, \mathrm{MEXU}$,

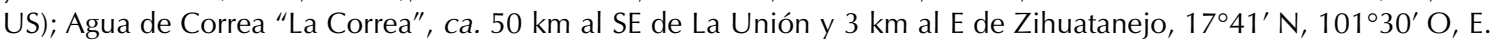
Langlassé $373(\mathrm{GH}) ; 3 \mathrm{~km}$ al NO de Vallecitos de Zaragoza, 1757’ N, 10120' O, 600 m, J. C. Soto 11657 (MEXU). La

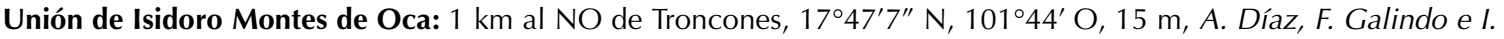

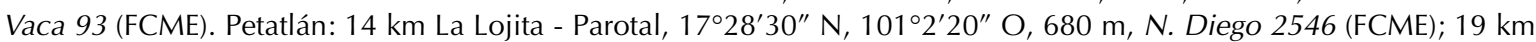
al N de Coyuquilla, $17^{\circ} 24^{\prime} \mathrm{N}, 101^{\circ} 2^{\prime} 50^{\prime \prime} \mathrm{O}, 260 \mathrm{~m}$, J. C. Soto, S. Román y A. Vidal 12160 (MEXU). Tlacochistlahuaca: ca. $3.5 \mathrm{~km}$ al S de San Pedro Cuitlapa, 16 $55^{\prime} 20^{\prime \prime}$ N, 98 $8^{\circ} 5^{\prime}$ O, 680 m, F. Lorea 4066 (FCME); 4.5 km al sur de San Isidro, 1653'22" N, 98¹8' O, 600 m, L. Lozada P. 1543 (FCME).

2. Aechmea mexicana Baker, J.Bot. 17: 165. 1879.

Figura 7

DISTRIBUCIÓN: México a Panamá.

ALTITUD: 1030-1900 m.

EJEMPLARES EXAMINADOS: Atoyac de Álvarez: cerca de Paraíso, $44 \mathrm{~km}$ al NE de Atoyac, $17^{\circ} 21^{\prime} \mathrm{N}, 100^{\circ} 14^{\prime} \mathrm{O}, 1030$

m, E. Martínez, J. C. Soto y B. Hernández 3747 (CHAP, MEXU). Coyuca de Catalán: La Primavera, 5 km al SE de Las Palancas, $17^{\circ} 47^{\prime} \mathrm{N}, 101^{\circ} 5^{\prime} \mathrm{O}, 1900$ m, F. González-Medrano 6801 (MEXU). General Heliodoro Castillo: en Las Golondrinas, 29 km al NE de Paraíso, 17²9’ N, 100¹' O, 1810 m, E. Martínez y T. P. Ramamoorthy 5203 (MEXU).

\section{Billbergia Thunb.}

3. Billbergia pallidiflora Liebm., Ann.Sci.Nat.Bot.sér.4. 2: 373. 1854.

Figura 7

DISTRIBUCIÓN: México a Nicaragua.

ALTITUD: ca. $200 \mathrm{~m}$.

EJEMPLARES EXAMINADOS: José Azueta: Agua de Correa "La Correa", ca. 50 km al SE de La Unión y 3 km al E de Zihuatanejo, $17^{\circ} 41^{\prime} \mathrm{N}, 101^{\circ} 30^{\prime} \mathrm{O}$, E. Langlassé 374 (US). La Unión de Isidoro Montes de Oca: ca. $21 \mathrm{~km}$ al N de Coyuquilla, rumbo a Infiernillo, $22 \mathrm{~km}$ al S de Infiernillo, por la carretera de cuota Lázaro Cárdenas - Uruapan, $18^{\circ}$ 5'37" N, 10155'2" O, 187 m, A. Espejo, A. R. López-Ferrari, J. Ceja, A. Mendoza e I. Ramírez 6601 (UAMIZ).

\section{BROMELIA L.}

1. Inflorescencia nidular, el escapo ausente o bien corto y escondido entre las hojas.

2. Sépalos estrechamente lanceolados, de 30 a $40 \mathrm{~mm}$ de largo; brácteas florales membranáceas, enteras o esparcidamente serradas; cápsulas maduras elipsoides a fusiformes.

2. Sépalos oblongos, de 25 mm de largo; brácteas florales papiráceas, delgadas, enteras; cápsulas maduras ovoides.

1. Inflorescencia escaposa, el escapo conspicuo.

3. Pétalos verdes; sépalos elíptico-oblongos, de 6 a $20 \mathrm{~mm}$ de largo, no más de tres veces tan largos como anchos; escapo delgado, con brácteas pequeñas, linear-lanceoladas.

3. Pétalos rojizos; sépalos muy angostamente triangular-subulados, de 15 a $30 \mathrm{~mm}$ de largo, 4 ó más veces más largos que anchos; escapo vigoroso con las brácteas muy angostamente triangular-subuladas, foliáceas, subinfladas B. pinguin

4. Bromelia hemisphaerica Lam., Encycl. 1:145. 1783.

Figura 8 DISTRIBUCIÓN: Endémica de México.

ALTITUD: $1100-1400 \mathrm{~m}$.

EJEMPLARES EXAMINADOS: Arcelia: Achotla, $18^{\circ} 19^{\prime} 30^{\prime \prime} \mathrm{N}, 100^{\circ} 16^{\prime} \mathrm{O}$, J. Jacques 35 (GH). Atlixtac: $1.37 \mathrm{~km}$ al NO de

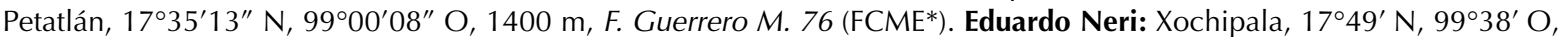
$1100 \mathrm{~m}$, C. Franco 16 (FCME, MEXU). 
5. Bromelia karatas L., Sp.PI. 285. 1753.

DISTRIBUCIÓN: México a Brasil y Las Antillas.

ALTITUD: 15-805 m.

EJEMPLARES EXAMINADOS: Chilpancingo de los Bravo: El Rincón, 45 km de Chilpancingo, camino a Acapulco,

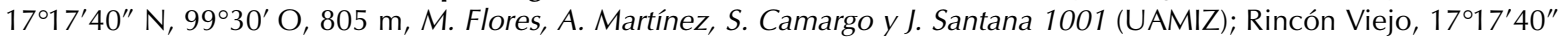
N, 99 $9^{\circ} 30^{\prime} \mathrm{O}, 750 \mathrm{~m}, \mathrm{H}$. Kruse 1246 (MEXU). Coyuca de Benítez: coast road 25 miles west of Acapulco, near Coyuca,

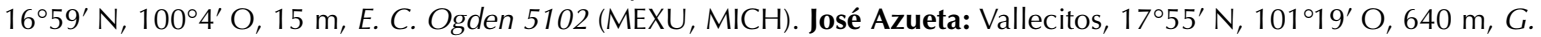
B. Hinton $10216(\mathrm{GH})$. La Unión de Isidoro Montes de Oca: puerta de la huerta de El Petatillo, $17^{\circ} 59^{\prime} \mathrm{N}, 101^{\circ} 48^{\prime} \mathrm{O}, E$. Guízar y Pimentel 2872 (CHAP, IEB, MEXU).

6. Bromelia palmeri Mez, in C.DC., Monogr.Phan. 9: 40. 1896.

Figura 8 DISTRIBUCIÓN: Endémica de México.

ALTITUD: 0-80 m.

EJEMPLARES EXAMINADOS: Acapulco de Juárez: parque nacional El Veladero, $16^{\circ} 55^{\prime} 50^{\prime \prime} \mathrm{N}, 9^{\circ} 53^{\prime} \mathrm{O}, \mathrm{N}$. Noriega A. 151 (FCME); isla La Roqueta, 1649'9" N, 9954'20" O, 0-80 m, J. Oseguera C. 106 (FCME). José Azueta: Zihuatanejo,

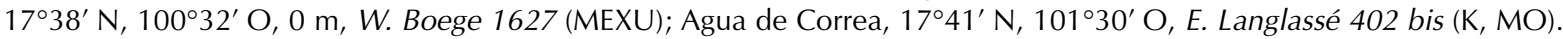
La Unión de Isidoro Montes de Oca: $1.6 \mathrm{~km}$ al SE de Troncones, $17^{\circ} 46^{\prime} 30^{\prime \prime} \mathrm{N}, 1^{\circ} 41^{\circ} 30^{\prime \prime} \mathrm{O}, 10 \mathrm{~m}$, A. Hanan, M. Gual y C. Gallardo 6 (FCME).

7. Bromelia pinguin L., Sp.PI. 285. 1753.

Figura 8

DISTRIBUCIÓN: México a Venezuela y Las Antillas.

ALTITUD: 0-150 m.

EJEMPLARES EXAMINADOS: Acapulco de Juárez: El Arenal, orilla de la laguna de Tres Palos, $16^{\circ} 47^{\prime} 40^{\prime \prime} \mathrm{N}, 9^{\circ} 42^{\prime} 55^{\prime \prime}$ O, 1 m, N. Diego 4144 (FCME); Punta Gorda, laguna de Tres Palos, 1645’45" N, 9942'20" O, 10 m, N. Diego 4975 (FCME); El Podrido, laguna de Tres Palos, 16 $16^{\circ} 45^{\prime} \mathrm{N}, 99^{\circ} 40^{\prime} 5^{\prime \prime} \mathrm{O}, 12 \mathrm{~m}$, S. Gil 200 (FCME); isla La Roqueta, 16 $46^{\circ} 49^{\prime} 16^{\prime \prime} \mathrm{N}$, 99 54'35" O, R. Otero, L. Radilla A. y A. G. Miranda R2 (CHAP). Atoyac de Álvarez, 45 miles W of Acapulco, near Cacalutla, $17^{\circ} 07^{\prime} 30^{\prime \prime} \mathrm{N}, 100^{\circ} 21^{\prime} 30^{\prime \prime} \mathrm{O}$, E. C. Ogden 5101 (MICH). Benito Juárez: rancho La Playa, Laguna de Mitla,

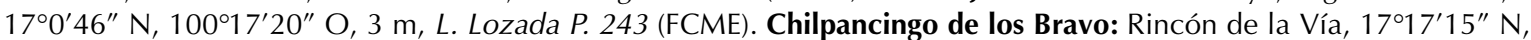

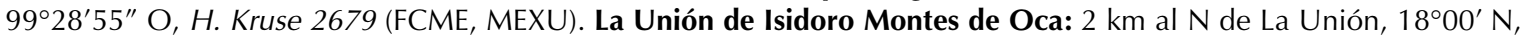

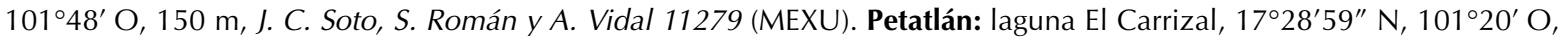

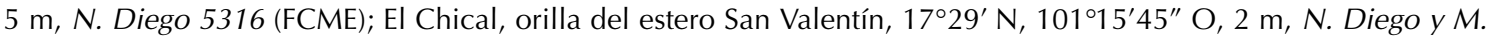

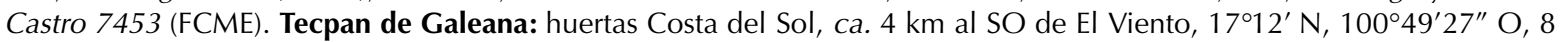
m, F. Lorea 5371 (FCME).

\section{CATOPSIS Griseb.}

1. Sépalos de (10) 13 a 20 mm de largo; pétalos espatulados de 15 a 25 mm de largo; cápsulas de (16) 19 a 24 de largo por 7 a $11 \mathrm{~mm}$ de diámetro.

1. Sépalos de 2 a $13 \mathrm{~mm}$ de largo; pétalos triangulares o estrechamente triangulares de 2 a $12 \mathrm{~mm}$ de largo; cápsulas de 8 a $15 \mathrm{~mm}$ de largo por 3 a $7 \mathrm{~mm}$ de diámetro.

2. Sépalos coriáceos, fuertemente carinados, lisos o muy levemente nervados cuando secos, de 7 a 13 $\mathrm{mm}$ de largo; raramente subcoriáceos y de 7 a $9 \mathrm{~mm}$ de largo en plantas estaminadas. C. berteroniana

2. Sépalos membranáceos o cartáceos, fuertemente nervados y rugosos cuando secos, de 4 a $8 \mathrm{~mm}$ de largo.

3. Brácteas del escapo de $1 / 4$ a $1 / 2$ de la longitud del entrenudo. C. sessiliflora

3. Brácteas del escapo más largas que los entrenudos, raramente las superiores más o menos iguales a ligeramente más cortas que los entrenudos.

4. Plantas en flor de (50) 70 a 100 (134) cm de alto; hojas de (25) 35 a $56 \mathrm{~cm}$ de largo; inflorescencia de 25 a $50 \mathrm{~cm}$ de largo: brácteas primarias inferiores triangular-lanceoladas, el ápice recto; cápsulas de (13) 15 a $17 \mathrm{~mm}$ de largo..... C. paniculata

4. Plantas en flor de 27 a $57 \mathrm{~cm}$ de alto; hojas de 17 a $30 \mathrm{~cm}$ de largo; inflorescencia de 9 a $25 \mathrm{~cm}$ de largo; brácteas primarias inferiores estrechamente triangulares, el ápice recurvado; cápsulas de 9 a $12 \mathrm{~mm}$ de largo 
FLora BROMELIOLÓGICA DE GUERRERO

8. Catopsis berteroniana (Schult. et Schult.f.) Mez, in C.DC., Monogr.Phan. 9: 621. 1896. DISTRIBUCIÓN: Estados Unidos, México, Las Antillas, Bahamas, Centro y Sudamérica.

ALTITUD: ca. $2400 \mathrm{~m}$.

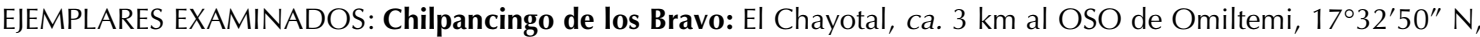
9941'02" O, 2500 m, E. Carranza y R. M. García 5299 (IEB); ca. 6 km después de Omiltemi, rumbo a Agua Fría, $17^{\circ} 33^{\prime} 22^{\prime \prime}$ N, 9944'47" O, 2420 m, A. R. López-Ferrari, A. Espejo y J. Ceja 2815 (UAMIZ).

9. Catopsis compacta Mez, Bull.Herb.Boissier sér. 2. 3:140. 1903.

Figura 9 DISTRIBUCIÓN: Endémica de México.

ALTITUD: $2200-2680 \mathrm{~m}$

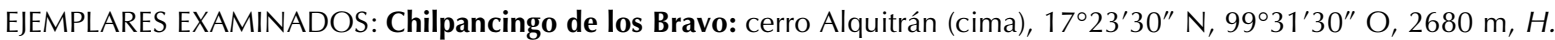

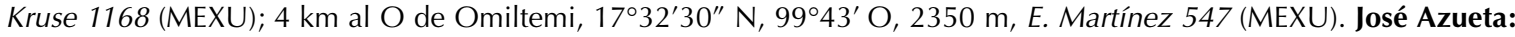

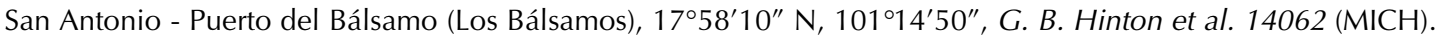

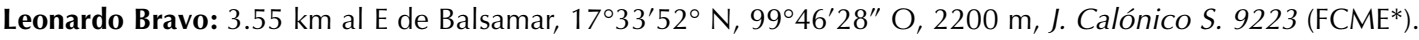

10. Catopsis nutans (Sw.) Griseb., Fl.Brit.W.I. 599. 1864.

Figura 9 DISTRIBUCIÓN: Estados Unidos, México, Las Antillas, Centro y Sudamérica. ALTITUD: $350-1880 \mathrm{~m}$. EJEMPLARES EXAMINADOS: Chilpancingo de los Bravo: Tres Caminos, $13 \mathrm{~km}$ al S rumbo a Jaleaca de Catalán, $17^{\circ} 26^{\prime} 60^{\prime \prime} \mathrm{N}, 99^{\circ} 52^{\prime} 04^{\prime \prime} \mathrm{O}, 750 \mathrm{~m}$, J. Calónico S. 8823 (FCME*); at km 338, $3 \mathrm{~km}$ beyond Acahuizotla, on hwy to Acapulco, $17^{\circ} 22^{\prime} \mathrm{N}, 9^{\circ} 27^{\prime} \mathrm{W}$, H. E. Moore y C. E. Wood 4686 (US ơ); en Jaleaca de Catalán, $17^{\circ} 27^{\prime} \mathrm{N}, 99^{\circ} 51^{\prime} \mathrm{O}, 790$

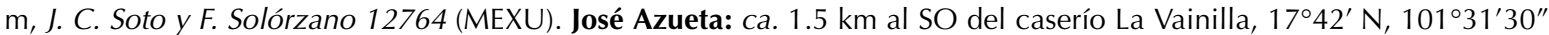
O, $350 \mathrm{~m}$, C. Gallardo, F. Lorea y A. Vargas 623 (FCME, MEXU). Leonardo Bravo: 8 km antes de llegar a Yextla, $17^{\circ} 32^{\prime} 41^{\prime \prime} \mathrm{N}, 99^{\circ} 54^{\prime} 55^{\prime \prime} \mathrm{O}, 1880$ m, B. González-Hidalgo1350 (FCME*). San Luis Acatlán: Mojonera de Atotonilco, 3 km al NO de Atotonilco, u 11 km al NO de Horcasitas, camino San Luis Acatlán - Pascala del Oro, 1655’ N, 9851' O, 800 m, E. Martínez, J. V. Lozano y B. Morales de Jesús 3552 (MEXU).

11. Catopsis paniculata E.Morren, in Jacob-Makoy, Cat.Hort. No. 121:1. X-1883.

Figura 9 DISTRIBUCIÓN: México a Nicaragua.

ALTITUD: $2300-2600 \mathrm{~m}$.

EJEMPLARES EXAMINADOS: Eduardo Neri: Cerro Ocotal, 3 km al SE de Amatitlán, 1750'56" N, 9944'36" O, 2300 m, R. Cruz-Durán y M. E. García G.791 (FCME); 10 km al E de Xochipala, 17²7’ N, 99³4' O, A. García-Mendoza, E. Martínez, Terrazas y Reyes 4859 (MEXU). General Heliodoro Castillo: $1 \mathrm{~km}$ adelante de La Vuelta, rumbo a Puerto del

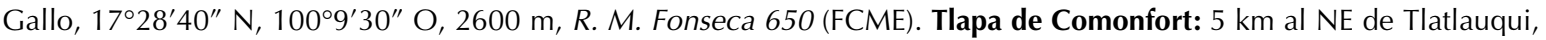
$17^{\circ} 34^{\prime} 5^{\prime \prime} \mathrm{N}, 98^{\circ} 47^{\prime} 30^{\prime \prime} \mathrm{O}$, J. I. Calzada 15859 (FCME), 15860 (FCME).

12. Catopsis sessiliflora (Ruiz et Pav.) Mez, in C.DC., Monogr.Phan. 9:625. 1896. DISTRIBUCIÓN: México, Las Antillas, Centro y Sudamérica.

ALTITUD: 900-2250 m.

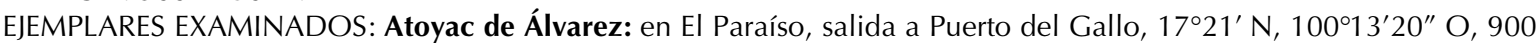

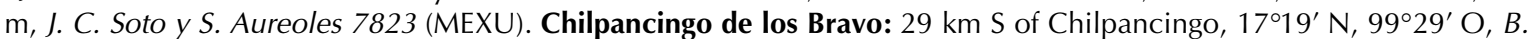

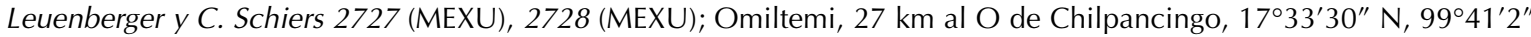
$\mathrm{O}, 2250 \mathrm{~m}$, Laboratorio de Biogeografía 18 (FCME).

\section{Fosterella L.B.SM.}

13. Fosterella micrantha (Lindl.) L.B.Sm., Phytologia 7:171, t. 1, f. 1-5. 1960.

Figura 7

DISTRIBUCIÓN: México a Guatemala y El Salvador.

ALTITUD: ca. $195 \mathrm{~m}$.

EJEMPLARES EXAMINADOS: Juan R. Escudero: Tierra Colorada, 179'56" N, 99³1'35" O, 195 m, H. Kruse 965 (ENCB, FCME, IEB, MEXU), 1001 (FCME, MEXU).

\section{HeCHTIA KLOTZSCH}

1. Margen de las hojas entero a serrado y entonces los dientes pequeños e inconspicuos, muy cercanos entre sí; rosetas con las hojas dísticamente dispuestas; flores azules a lilas. H. caerulea 


\section{Valeria Angélica Pulido-Esparza, Ana Rosa LóPEZ-Ferrari y Adolfo EsPejo-Serna}

1. Margen de la hojas con espinas uncinadas y conspicuas, separadas unas de otras por más de $5 \mathrm{~mm}$; rosetas con las hojas polísticamente dispuestas; flores blancas a amarillentas o verdosas.

2. Plantas femeninas (flores pistiladas y/o frutos maduros presentes).

3. Inflorescencia tripinnada; tépalos con puntos negros; cápsulas maduras de 5 a $6 \mathrm{~mm}$ de largo.... H. sphaeroblasta

3. Inflorescencia simple o bipinnada, raramente tripinnadas en las ramas basales; tépalos sin puntos negros; cápsulas maduras de (6-) 7 a $14 \mathrm{~mm}$ de largo.

4. Ramas de la inflorescencia de (1-) 2 a 7 (-10) $\mathrm{cm}$ de largo.

5. Rosetas largamente caulescentes, con frecuencia sobrepasando los $2 \mathrm{~m}$ de largo; ramas de la inflorescencia de 5 a $6.5 \mathrm{~cm}$ de largo; brácteas florales más cortas que los pedicelos; cápsulas de 8 a $10 \mathrm{~mm}$ de largo por 3 a $4 \mathrm{~mm}$ de diámetro.

H. mooreana

5. Rosetas compactas y acaulescentes; ramas de la inflorescencia de 2 a $3 \mathrm{~cm}$ de largo; brácteas florales sobrepasando a los pedicelos y casi totalmente a los sépalos; cápsulas de 6 a $11 \mathrm{~mm}$ de largo por 3 a $5 \mathrm{~mm}$ de diámetro....

H. pumila

4. Ramas de la inflorescencia de 9 a $35 \mathrm{~cm}$ de largo.

6. Cápsulas maduras casi negras, lustrosas, ascendentes; ramas de la inflorescencia de 20 a $35 \mathrm{~cm}$ de largo H. melanocarpa

6. Cápsulas maduras pardas a grisáceas, opacas, divaricadas a descendentes; ramas de la inflorescencia de 9 a $17 \mathrm{~cm}$ de largo.

7. Inflorescencia ramificada, las ramas más de dos; cápsulas lanceoloides, de 9 a $11 \mathrm{~mm}$ de largo por $4 \mathrm{~mm}$ de diámetro, divaricadas, pardas; pedicelos en el fruto de 4 a $6 \mathrm{~mm}$ de largo por $0.6 \mathrm{~mm}$ de diámetro H. reflexa

7. Inflorescencia simple o raramente con una o dos ramas basales; cápsulas ovoides, de 12 a $14 \mathrm{~mm}$ de largo por 2 a 4 mm de diámetro, descendentes, grisáceas; pedicelos en el fruto de 1.5 a $4 \mathrm{~mm}$ de largo por $2 \mathrm{~mm}$ de diámetro..... H. carlsoniae

2. Plantas masculinas (flores estaminadas; frutos ausentes).

8. Inflorescencia tripinnada, al menos en las ramas inferiores.

9. Pétalos blancos, de 2.7 a $3 \mathrm{~mm}$ de largo por ca. $2 \mathrm{~mm}$ de ancho en la base; sépalos de ca. $1.8 \mathrm{~mm}$ de largo por 1.7 a $2 \mathrm{~mm}$ de ancho en la base; filamentos de 2.7 a $2.8 \mathrm{~mm}$ de largo; anteras de 1 a $1.2 \mathrm{~mm}$ de largo. H. sphaeroblasta

9. Pétalos verdes a verde amarillentos, de 4.5 a $4.6 \mathrm{~mm}$ de largo por ca. $2.8 \mathrm{~mm}$ de ancho en la base; sépalos de 2.7 a $2.8 \mathrm{~mm}$ de largo por ca. $2.8 \mathrm{~mm}$ de ancho en la base; filamentos de 3.9 a $4 \mathrm{~mm}$ de largo; anteras de 2.4 a $2.5 \mathrm{~mm}$ de largo...... H. melanocarpa

8. Inflorescencia bipinnada o bien simple.

10. Ramas de la inflorescencia de (8-) 12 a $25 \mathrm{~cm}$ de largo.

11. Pedicelos en la antesis de ca. $2 \mathrm{~mm}$ de largo, delgados H. reflexa

11. Pedicelos en la antesis de menos de $0.5 \mathrm{~mm}$, robustos, o bien ausentes.

12. Pétalos verdes a verde amarillentos; plantas de más de $1 \mathrm{~m}$ de alto; hojas de más de $40 \mathrm{~cm}$ de largo.

12. Pétalos blancos; plantas de menos de $90 \mathrm{~cm}$ de alto; hojas de menos de $30 \mathrm{~cm}$ de largo...... H. carlsoniae

10. Ramas de la inflorescencia de 3 a $10(-14) \mathrm{cm}$ de largo.

13. Rosetas largamente caulescentes, con frecuencia sobrepasando los $2 \mathrm{~m}$ de largo; pedicelos alargados en antesis; brácteas más cortas que los pedicelos. H. mooreana

13. Rosetas compactas y acaulescentes; pedicelos muy cortos en antesis; brácteas sobrepasando los pedicelos y casi totalmente a los sépalos. 
14. Hechtia caerulea (Matuda) L.B.Sm., Phytologia 24: 446, t. 5, f. 5.1972.

Figura 10 DISTRIBUCIÓN: Endémica de México.

ALTITUD: ca. $1100 \mathrm{~m}$.

EJEMPLARES EXAMINADOS: Pilcaya: Grutas de Cacahuamilpa, 1840’ N, 99³0’ O, ca. 1100 m, J. Vázquez S. 3139 (MEXU); A. Espejo s. n. (Diapositivas UAMIZ).

15. Hechtia carlsoniae Burt-Utley et Utley, Syst.Bot. 13:276-279, f. 1, t. 1. 1988. DISTRIBUCIÓN: Endémica de México.

ALTITUD: 810-1000 m.

EJEMPLARES EXAMINADOS: Buenavista de Cuéllar: Cañón de la Mano, 18²4'36" N, 99³1'13" O, $810 \mathrm{~m}$, A. R. López y A. Espejo 2844 O (UAMIZ), 2845 O (UAMIZ). Taxco de Alarcón: 0.8 miles $\mathrm{N}$ of Mexcaltepec, $0.9 \mathrm{miles} \mathrm{N}$ of km 112 marker and 2.1 miles $\mathrm{N}$ of El Naranjo along Mex 95 from Taxco to Iguala, $18^{\circ} 27^{\prime} \mathrm{N}, 9^{\circ} 33^{\prime} \mathrm{W}, 3200 \mathrm{ft}$, J. Utley y K. Burt Utley 7492 (MEXU).

OTROS REGISTROS: Buenavista de Cuéllar: Cañón de la Mano, 18²45'ㄹ N, 99²9'50" O, M. C. Carlson 3035 (F). [Burt Utley y Utley, 1988: 277].

16. Hechtia melanocarpa L.B.Sm., Contr.Gray Herb. 161:32, t. 4, f. 8-9. 1946.

Figura 10 DISTRIBUCIÓN: Endémica de México.

ALTITUD: 565-1250 m.

EJEMPLARES EXAMINADOS: Chilpancingo de los Bravo: km 4.5 carretera a Chilpancingo, $17^{\circ} 35^{\prime} 00^{\prime \prime} \mathrm{N}^{\prime} 9^{\circ} 32^{\prime} 00^{\prime \prime} \mathrm{O}$, H. Kruse 2690 (FCME, MEXU). Copalillo: $1.5 \mathrm{~km}$ al NO del poblado de Papalutla, $18^{\circ} 02^{\prime} \mathrm{N}, 9^{\circ} 54^{\prime} 50^{\prime \prime} \mathrm{O}, 820 \mathrm{~m}, \mathrm{M}$. A.

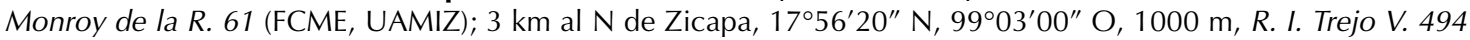

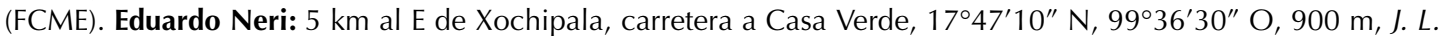
Contreras J. 2410 (FCME); Xochipala, camino a Papalotepec, $17^{\circ} 49^{\prime} \mathrm{N}, 99^{\circ} 38^{\prime} \mathrm{O}, 1050 \mathrm{~m}, \mathrm{C}$. Franco 3 (MEXU), 6

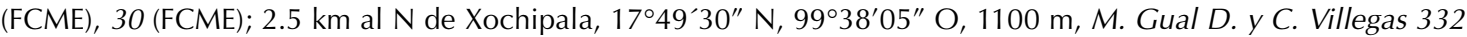

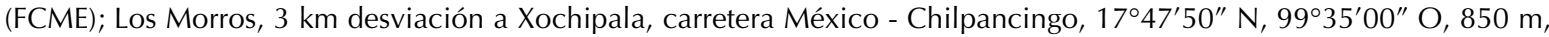
S. Peralta G. y C. Villegas 163 (FCME); cerro Papalotepec, $17^{\circ} 45^{\prime} 20^{\prime \prime}$ N, 99 $37^{\prime} \mathrm{O}, 1250 \mathrm{~m}$, S. Peralta G. y C. Villegas 219 (FCME); 12 km al S de Mezcala, 1750’ N, 19³3’30” O, 565 m, L. Rico, E. Martínez y O. Téllez 422 (MEXU, TEX);

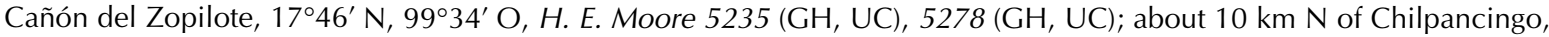
$17^{\circ} 46^{\prime} \mathrm{N}, 9^{\circ} 34^{\prime} \mathrm{W}$, E. C. Ogden $5112(\mathrm{MICH})$; Mezcala, 1756 $\mathrm{N}, 9^{\circ} 36^{\prime} \mathrm{O}$, W. Schwabe s. n. (MEXU). Mártir de

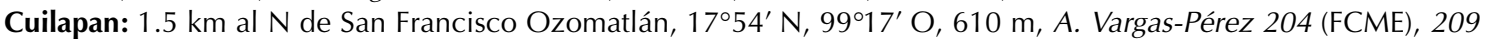

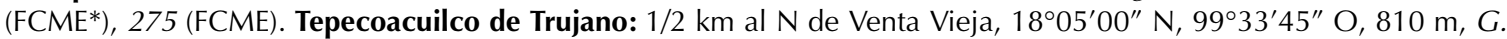
Campos R., E. Castelo N., E. Velázquez M. y C. Toledo 12 (FCME); 5.5 km al NNE de Mezcala, 1759'30" N, 99³5'15" O, 750 m, E. Castelo N. y G. Campos R. 39 (FCME); 3 km al NE de la desviación a Mezcala, carretera México -

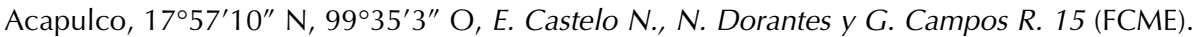

OTROS REGISTROS: Eduardo Neri: Cañón del Zopilote $17^{\circ} 46^{\prime} \mathrm{N}, 9^{\circ} 34^{\prime} \mathrm{O}, \mathrm{M}$. B. Foster 1258 (GH) [Smith y Downs, 1974: 582]; F. Miranda 9306 [Burt-Utley y Utley, 1988: 282]; J. Utley y K. Burt-Utley 7493, 7494, 7499 [Burt-Utley y Utley, 1988: 281].

17. Hechtia mooreana L.B.Sm., Contr.U.S.Natl.Herb. 29:522-523, f. 78. 1954.

Figura 11 DISTRIBUCIÓN: Endémica de México.

ALTITUD: $520-1250 \mathrm{~m}$.

EJEMPLARES EXAMINADOS: Chilapa de Álvarez: ca. $5 \mathrm{~km}$ al NE de Zoquiapan y $1 \mathrm{~km}$ al S de La Pera (EI Peral), $F$.

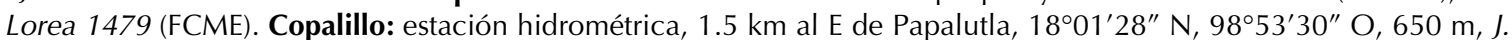
Calónico S. 829 (FCME). Eduardo Neri: 2.5 km al NE del El Palmar, 17³9’38” N, 99³4'48” O, 1200 m, J. Calónico S.

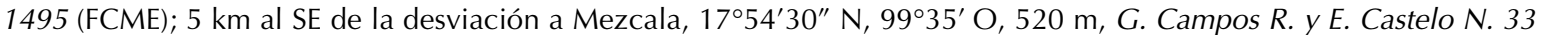
(FCME); Cañón del Zopilote, parte baja, $17^{\circ} 46^{\prime} \mathrm{N}, 9^{\circ} 34^{\prime} \mathrm{O}$, E. Castelo N. y G. Campos R. 40 (FCME); Xochipala

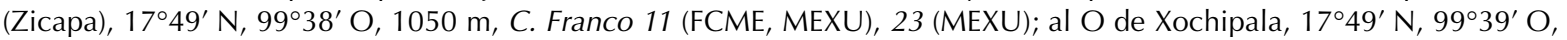

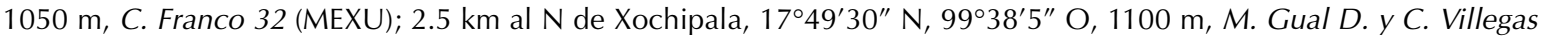
s. n. (FCME); Barranca del Tecolote, Xochipala, $17^{\circ} 49^{\prime} \mathrm{N}, 9^{\circ} 37^{\prime} 40^{\prime \prime} \mathrm{O}, 900 \mathrm{~m}$, M. Gual D. y C. Villegas 290 (FCME); 3 km al NE de Xochipala, 1749'45" N, 99³8' O, 880 m, M. Gual D. y C. Villegas 291 (FCME); Cañón del Zopilote

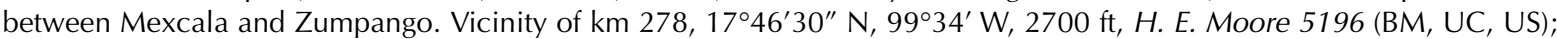
cerro Papalotepec, $17^{\circ} 45^{\prime} 20^{\prime \prime} \mathrm{N}$, 99 $9^{\circ} 37^{\prime} \mathrm{O}, 1250 \mathrm{~m}$, S. Peralta G. y C. Villegas 218 (FCME); Cañón del Zopilote, cerca de Milpillas, $17^{\circ} 46^{\prime} \mathrm{N}, 99^{\circ} 34^{\prime} \mathrm{O}, 750 \mathrm{~m}$, J. Rzedowski 22627 (GH, IEB, MEXU, MO); 8 km al S de Axaxacoalco, $17^{\circ} 45^{\prime} 50^{\prime \prime} \mathrm{N}, 9^{\circ} 28^{\prime} 41^{\prime \prime} \mathrm{O}, 1160 \mathrm{~m}, \mathrm{~S}$. Valencia A. 1205 (FCME, MEXU). Mártir de Cuilapan: $1.5 \mathrm{~km}$ al E de San

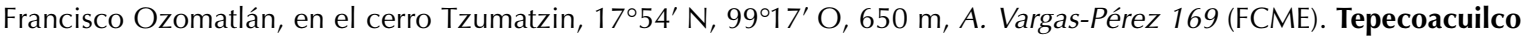


Valeria Angélica Pulido-Esparza, Ana Rosa LóPeZ-Ferrari y Adolfo EsPejo-Serna

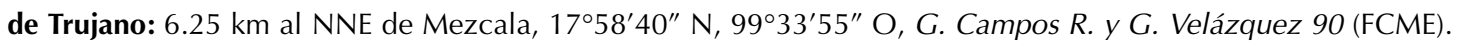

18. Hechtia pumila Burt-Utley et Utley, Syst.Bot. 13:279-281, f. 2. 1988.

Figura 11

DISTRIBUCIÓN: Endémica de México.

ALTITUD: $610-1650 \mathrm{~m}$.

EJEMPLARES EXAMINADOS: Chilpancingo de los Bravo: about 2.6 miles S of Petaquillas along Mex 95 from

Chilpancingo to Acapulco, $17^{\circ} 28^{\prime} \mathrm{N}, 99^{\circ} 28^{\prime} \mathrm{W}, 4400 \mathrm{ft}, \mathrm{K}$. Burt-Utley y Utley 7902 (MEXU); Mazatlán, falda E del cerro Alquitrán, $17^{\circ} 26^{\prime} 01^{\prime \prime} \mathrm{N}, 9^{\circ} 27^{\prime} 54^{\prime \prime} \mathrm{O}, 1650 \mathrm{~m}$, H. Kruse 1171 (FCME); dry hills with lime rock near km 315 on hwy

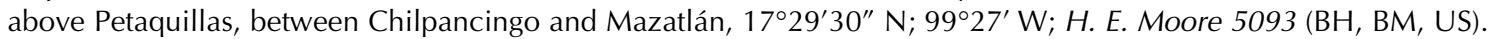

Mártir de Cuilapan: paraje Cerro de Achichican, terrenos del ejido San Agustín Oapan, 1756 $02^{\prime \prime}$ N, 99²5’50" O, 610 m, E. Guízar N. 4991 (MEXU).

OTROS REGISTROS: Chilpancingo de los Bravo: Chilpancingo, 17³3’ N, 99॰30’ O, C. M. Rowell, J. Paxson y F. A. Barkley 17-M-879 (F, TEX) [Smith y Downs, 1974: 597; Burt-Utley y Utley, 1988: 281]; near Chilpancingo, 17³2'58" N, 99²9'45" W, J. Utley y K. Burt-Utley 7614 ö y o (BH, AS, F, GH, MICH, MO, NY, US) [Burt-Utley y Utley, 1988: 281].

19. Hechtia reflexa L.B.Sm., Contr.Gray Herb. 117:18-19, t. 1, f. 54, 55. 1937.

Figura 10 DISTRIBUCIÓN: Endémica de México.

ALTITUD: $100-350 \mathrm{~m}$.

EJEMPLARES EXAMINADOS: La Unión de Isidoro Montes de Oca: cerca de La Garita, 8 km al SE del Infiernillo, carretera

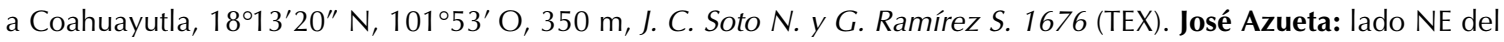

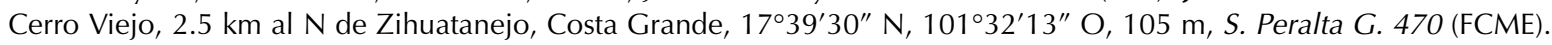

20. Hechtia sphaeroblasta B.L.Rob., Proc.Amer.Acad.Arts 35 (Contr. Gray Herb. n. s. 18): 323. 1900.

DISTRIBUCIÓN: Endémica de México.

ALTITUD: $700-1620 \mathrm{~m}$.

EJEMPLARES EXAMINADOS: Alpoyeca: $1.5 \mathrm{~km}$ al E de Tecoyo, cañada Tecuane, 17³5'29" N, 98²8'28" O, $1000 \mathrm{~m}, J$. Calónico S. 1671 (FCME); $1.5 \mathrm{~km}$ al N de Tecoyo, 17³6'10" N, 98²9'29" O, 1070 m, J. Calónico S. 1913 (FCME).

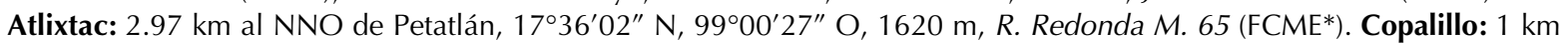
al S de Tlalcozotitlán, 1752'50" N, 9908'00" O, 930 m, G. Lozano V. 30 (FCME), R. I. Trejo V. y E. López 420 (FCME).

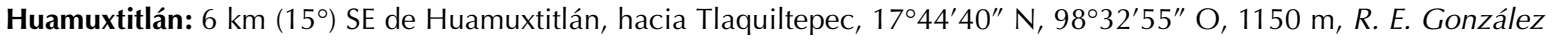
F. 56 (FCME*); 3 km al S de Humauxtitlán, camino Tlapa, 17046'1" N, 98³3’ O, 960 m, E. Martínez 1031 (MEXU); 5 km

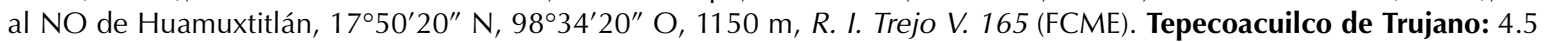
$\mathrm{km}\left(45^{\circ}\right) \mathrm{SE}$ de Palula hacia Ahuelican, $18^{\circ} 04^{\prime} 00^{\prime \prime} \mathrm{N}, 99^{\circ} 31^{\prime} 40^{\prime \prime} \mathrm{O}, 850 \mathrm{~m}, \mathrm{~V}$. Aguilar s. n. (FCME); $49 \mathrm{~km}$ al S de Iguala, $4 \mathrm{~km}$ al S de Xalitla, carretera Iguala - Chilpancingo, $17^{\circ} 58^{\prime} 30^{\prime \prime} \mathrm{N}, 99^{\circ} 34^{\prime} \mathrm{O}$, J. C. Soto y S. Aureoles $8853 \mathrm{f}$ (MEXU).

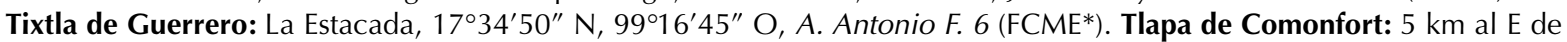

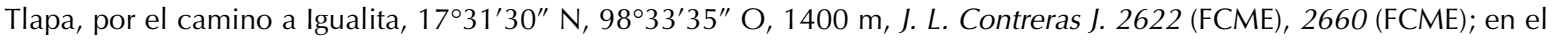

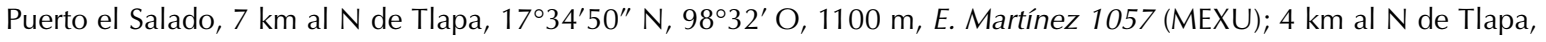
sobre la carretera Tlapa - Alpoyeca, $17^{\circ} 34^{\prime} \mathrm{N}, 98^{\circ} 33^{\prime} \mathrm{O}, 1080 \mathrm{~m}$, M. Flores C, A. Martínez B., J. Santana C. y $S$. Camargo 937 (UAMIZ); 4 km al N de Tlapa, sobre la carretera Tlapa - Alpoyeca, 17³4' N, 98³3' O, $1080 \mathrm{~m}$, M. Flores C, A. Martínez B., J. Santana C. y S. Camargo 937-bis (UAMIZ); cerro cerca de Los Otates, ca. $15 \mathrm{~km}$ al E de Tlapa,

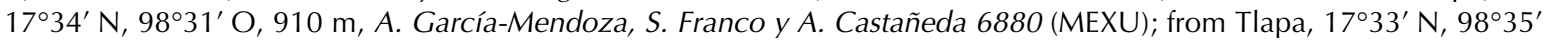

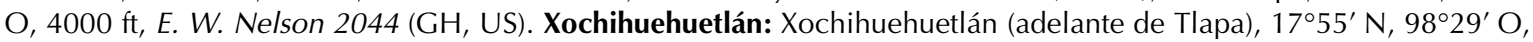
$1300 \mathrm{~m}$, E. Matuda y colaboradores 38471 (MEXU, US); paraje Ocotepec, $2.75 \mathrm{~km}$ al NNO de Jilotepec, cerro Xilotzin, $17^{\circ} 58^{\prime} 19^{\prime \prime} \mathrm{N}, 98^{\circ} 30^{\prime} 23^{\prime \prime} \mathrm{O}, 1410 \mathrm{~m}$, E. Moreno G. et al. 726 (FCME).

\section{Pitcairnia L’Hér.}

1. Hojas persistentes, con frecuencia pecioladas; plantas mesófilas.

2. Brácteas florales grandes, de más de $2 \mathrm{~cm}$ de largo, imbricadas, más largas que los sépalos; hojas conspicuamente pecioladas...... P. imbricata

2. Brácteas florales pequeñas, de menos de $1 \mathrm{~cm}$ de largo, más cortas que los sépalos y que los pedicelos; hojas angostándose hacia la base pero no conspicuamente pecioladas.

3. Plantas de hasta $50 \mathrm{~cm}$ de alto; láminas de las hojas de 2 a $2.7 \mathrm{~cm}$ de ancho; brácteas florales de $5 \mathrm{~mm}$ de largo; pétalos rojos, de ca. $7 \mathrm{~cm}$ de largo...... P. oaxacana

3. Plantas de 60 a $70 \mathrm{~cm}$ de alto; láminas de las hojas de (3-) 4 a $5 \mathrm{~cm}$ de largo; brácteas florales de 7 
a $9 \mathrm{~mm}$ de largo; pétalos amarillos, de 4 a $5 \mathrm{~cm}$ de largo.

P. flavescentia

1. Hojas deciduas a lo largo de una línea transversal, generalmente sésiles; plantas xerófilas.

4. Escapo de la inflorescencia muy corto o ausente; pétalos provistos de una escama basal interna

P. heterophylla

4. Escapo de la inflorescencia conspicuo; pétalos sin una escama basal interna.

5. Pedicelos de menos de $2 \mathrm{~mm}$ de largo.

6. Bráctas florales glabras a glabrescentes en la antesis.

P. cylindrostachya

6. Brácteas florales y raquis densa y persistentemente blanco lepidotos.

P. roseana

5. Pedicelos de 4 a $20 \mathrm{~mm}$ de largo.

7. Pétalos de 7 a $10 \mathrm{~cm}$ de largo; sépalos de 3 a $3.5 \mathrm{~cm}$ de largo.

8. Cálices densamente tomentosos, aterciopelados, el tomento pardo oscuro a ferrugíneo

P. modesta

8. Cálices glabros a glabrescentes, el indumento cuando presente blanquecino a pardo claro.

9. Sépalos esparcidamente lepidotos, las escamas estrellado-blanquecinas a pardo claras.

P. leprosa

9. Sépalos glabros P. hintoniana

7. Pétalos de 4 a $7 \mathrm{~cm}$ de largo; sépalos de 1.5 a $2.4 \mathrm{~cm}$ de largo.

9. Brácteas florales más cortas que los pedicelos; flores de $7 \mathrm{~cm}$ de largo.

P. mooreana

9. Brácteas florales del mismo tamaño o más largas que los pedicelos; flores de 4 a $5 \mathrm{~cm}$ de largo.

10. Hojas monomórficas; inflorescencia con 3 a 5 flores laxamente dispuestas. P. tillandsioides

10. Hojas dimórficas; inflorescencia con más de 10 flores más o menos apretadamente dispuestas.

11. Flores secundas; pedicelos de 10 a $14 \mathrm{~mm}$ de largo, aplanados. P. pteropoda

11. Flores polísticas; pedicelos de 5 a $9 \mathrm{~mm}$ de largo, rollizos.

12. Inflorescencia tomentosa, de aspecto sucio o polvoriento; sépalos posteriores no alados P. sordida

12. Inflorescencia glabra; sépalos posteriores conspicuamente alados. P. karwinskyana

21. Pitcairnia cylindrostachya L.B.Sm., Contr.Gray Herb. 117:25, t. 2, f. 14, 15. 1937. DISTRIBUCIÓN: Endémica de México.

ALTITUD: ca. $1810 \mathrm{~m}$.

EJEMPLARES EXAMINADOS: General Heliodoro Castillo: en Las Golondrinas, $29 \mathrm{~km}$ al NE de El Paraíso, 17⒉ $29^{\prime}$ N, $100^{\circ} 1^{\prime}$ O, 1810 m, J. C. Soto, E. Martínez y T. P. Ramammorthy 5220 (MEXU).

22. Pitcairnia flavescentia Matuda, Anales Inst.Biol.Univ.Nac.México 36: 110-111, f. 5. 1966.

Figura 11 DISTRIBUCIÓN: Endémica de México.

ALTITUD: $800-900 \mathrm{~m}$

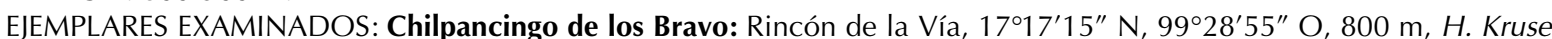

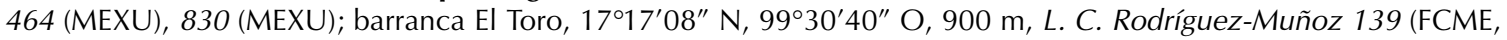
UAMIZ); camino Soyaltepec - Ocotito (3), 17018'40" N, 99³4' O, C. Verduzco M. 445 (FCME, UAMIZ), 446 (FCME*), 446 B (UAMIZ).

23. Pitcairnia heterophylla (Lindl.) Beer, Fam.Bromel. 68. 1857.

Figura 11 DISTRIBUCIÓN: México a Colombia y Venezuela.

ALTITUD: $1600-1900 \mathrm{~m}$.

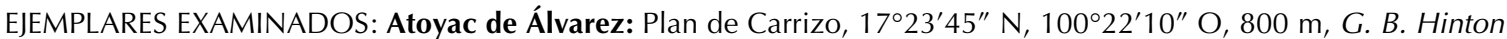
11027 (GH, US); ca. $14 \mathrm{~km}$ al SSO del campamento El Gallo, sobre el camino a Atoyac, estribaciones suroccidentales del cerro Teotepec, $17^{\circ} 25^{\prime} \mathrm{N}, 100^{\circ} 14^{\prime} \mathrm{O}, 1600-1900 \mathrm{~m}$, J. Rzedowski y R. McVaugh 72 (ENCB, MEXU, MICH).

Chilpancingo de los Bravo: in mountains above Mazatlán, $17^{\circ} 26^{\prime} 30^{\prime \prime} \mathrm{N}, 9^{\circ} 28^{\prime} \mathrm{W}$, D. S. Correll 14388 (LL, US). 
24. Pitcairnia hintoniana L.B.Sm., Contr.Gray Herb. 114:7, t. 1, f. 8, 9. 1936.

Figura 12 DISTRIBUCIÓN: Endémica de México.

ALTITUD: $1500 \mathrm{~m}$.

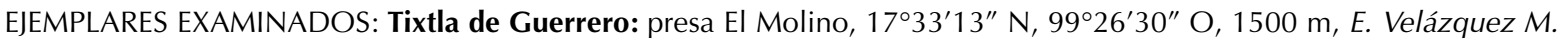
1526 (FCME, UAMIZ).

25. Pitcairnia imbricata (Brongn.) Regel, Gartenflora 17: 135, t. 579. 1868.

Figura 12

DISTRIBUCIÓN: México a Nicaragua.

ALTITUD: 850-1500 m.

EJEMPLARES EXAMINADOS: Atoyac de Álvarez: 8 km antes de El Edén, sobre la desviación... (sic), $17^{\circ} 22^{\prime} 30^{\prime \prime} \mathrm{N}$, $100^{\circ} 11^{\prime} 30^{\prime \prime} \mathrm{O}, 1210 \mathrm{~m}$, V. C. Aguilar J. 642 (FCME*); en El Ranchito, $11 \mathrm{~km}$ al NE de El Paraíso, $17^{\circ} 23^{\prime} \mathrm{N}, 1^{\prime} 0^{\circ} 12^{\prime} \mathrm{O}^{\prime}$ $1100 \mathrm{~m}$, J. C. Soto y F. Solórzano 12815 (MEXU, UAMIZ); $36 \mathrm{~km}$ al SO de Puerto del Gallo, 17¹5' N, 100²2' O, 1500 $\mathrm{m}$, P. Tenorio, E. Martínez y C. Romero de T. 487 (MEXU, MO). Chilpancingo de los Bravo: Agua de Obispo, ladera E del

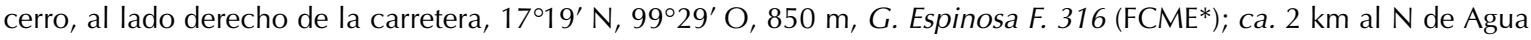

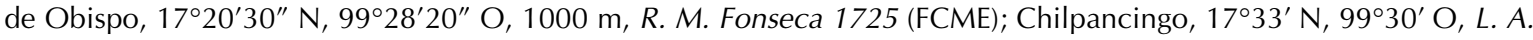

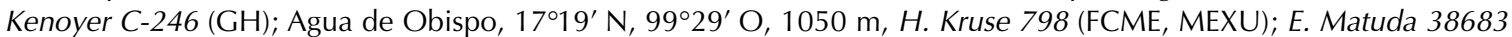
(ENCB, FCME, MEXU), 38711 (MEXU); km 339-340 on Acapulco highway, Acahuizotla to Agua de Obispo, $17^{\circ} 22^{\prime} \mathrm{N}$, 99²8' W, H. E. Moore y C. E. Wood 4705 (AA, MICH, US), H. E. Moore 5112 (GH, UC, US); ravine N of road along stream in back of road camp, $\mathrm{km}$ 304-305 on highway below Acahuizotla, between Chilpancingo and Acapulco, $17^{\circ} 22^{\prime}$ $\mathrm{N}, 99^{\circ} 29^{\prime} \mathrm{W}, \mathrm{H}$. E. Moore 8130 (MEXU, US). Tlacochistlahuaca: $c a .1 \mathrm{~km}$ al SE de Mixtecolapa, sobre el camino Tres Cruces - Pascala del Oro, $17^{\circ} 01^{\prime} \mathrm{N}, 98^{\circ} 06^{\prime} 40^{\prime \prime} \mathrm{O}$, F. Lorea 4884 (FCME).

26. Pitcairnia karwinskyana Schult. et Schult.f., Syst.Veg. 7: 1239-1240. 1830.

Figura 13 DISTRIBUCIÓN: Endémica de México.

ALTITUD: 700-1740 m.

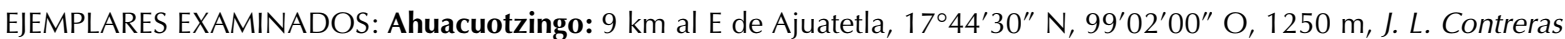

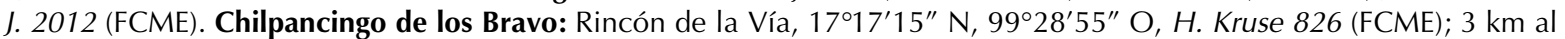
SE de Acahuizotla, $17^{\circ} 21^{\prime} \mathrm{N}, 9^{\circ} 27^{\prime} 30^{\prime \prime} \mathrm{O}, 700 \mathrm{~m}$, A. Nuñez 703 (FCME, UAMIZ); 25 miles S of Chilpancingo, 17³3' N, 99³0’ W, J. Paxson, G. Webster y F. A. Barkley 17-M-793 (LL). Coyuca de Catalán: Trincheras, $18^{\circ} 05^{\prime} \mathrm{N}, 1^{\circ} 1^{\circ} 16^{\prime} \mathrm{O}$,

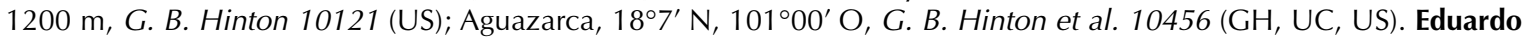
Neri: La Yesera, $12.3 \mathrm{~km}$ al N de Zumpango del Río, 1742’30" N, 99³0’00" O, $1500 \mathrm{~m}$, J. Calónico S. 1089 bis (FCME). Malinaltepec: Malinaltepec, $17^{\circ} 15^{\prime} \mathrm{N}, 98^{\circ} 40^{\prime} 25^{\prime \prime} \mathrm{O}, 1500 \mathrm{~m}, \mathrm{l}$. Wagenbreth 676 (MEXU, MO). Olinalá: 14 km al E de Papalutla, crucero Olinalá-Xixila-Papalutla, $18^{\circ} 01^{\prime} 40^{\prime \prime} \mathrm{N}, 98^{\circ} 46^{\prime} 30^{\prime \prime} \mathrm{O}, 1500 \mathrm{~m}$, M. A. Monroy de la R. 146 (FCME). Pilcaya: El Mogote, 1840'58" N, 99³3'46" O, R. Cruz-Durán 2429 (FCME). Taxco de Alarcón: N of Taxco,

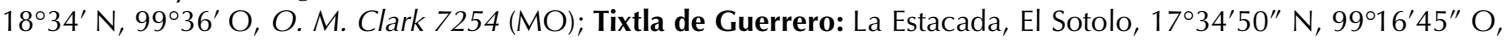

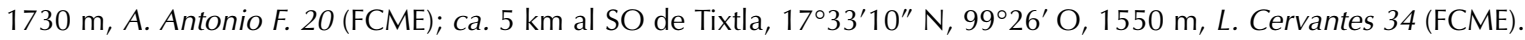

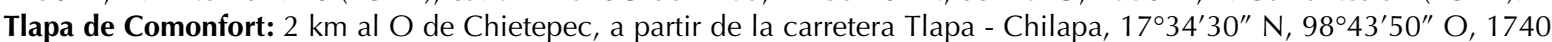
m, J. Santana C. 709 (FCME). Zirándaro: 12.4 km al O de Los Parajes, 18¹1'01" N, 101¹2'01" O, 1530 m, J. Calónico S. y R. Mayorga S. 15344 (FCME).

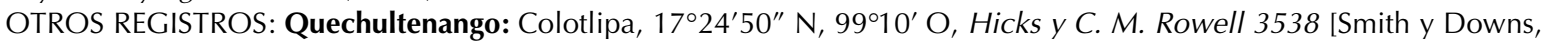
1974: 431], como P. puberula.

27. Pitcairnia leprosa L.B.Sm., Contr.Gray Herb. 161:33, t. 4, f. 4. 1946.

Figura 12 DISTRIBUCIÓN: Endémica de México.

ALTITUD: $520-800 \mathrm{~m}$.

EJEMPLARES EXAMINADOS: Coahuayutla de José María Izazaga: El Aguacate, $5.33 \mathrm{~km}$ al S, $18^{\circ} 7^{\prime} 31^{\prime \prime} \mathrm{N}, 101^{\circ} 27^{\prime} 34^{\prime \prime}$ O, 800 m, J. Calónico y M. Antonio 16251 (FCME, MEXU). Coyuca de Catalán: Zapo "Sapo", 1755' N, 1010' O, G. B. Hinton 10653 (US). José Azueta: Vallecitos, $17^{\circ} 55$ N, 101¹9' O, 520 m, G. B. Hinton et al. 10552 (GH, MEXU, NY, US).

28. Pitcairnia modesta L.B.Sm., Phytologia 7:417-418, t. 1, f. 1, 2. 1961.

Figura 12 DISTRIBUCIÓN: Endémica de México.

ALTITUD: 300-1000 m.

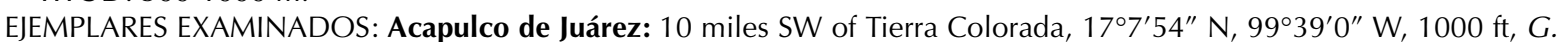

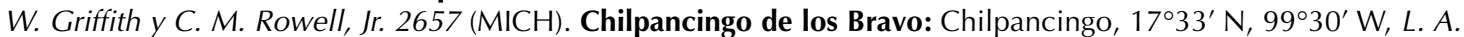

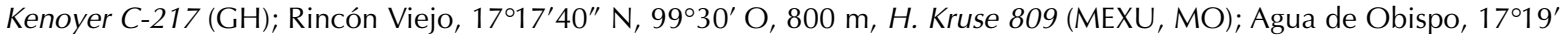

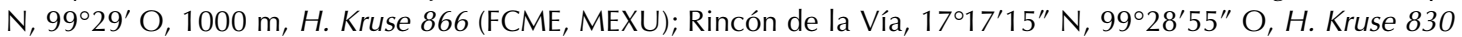


FLORA BROMELIOLÓGICA DE GUERRERO

(MEXU), 867 (FCME, MEXU); 25 miles S of Chilpancingo, $17^{\circ} 25^{\prime} \mathrm{N}, 9^{\circ} 28^{\prime} \mathrm{W}$, J. Paxson, G. Webster y F. A. Barkley $17-$ M-819 (LL); $5.2 \mathrm{~km}$ al O del Ocotito, camino a Jaleaca, $17^{\circ} 16^{\prime} \mathrm{N}, 9^{\circ} 33^{\prime} 30^{\prime \prime} \mathrm{O}, 735 \mathrm{~m}$, R. Torres, E. Martínez, P. Tenorio

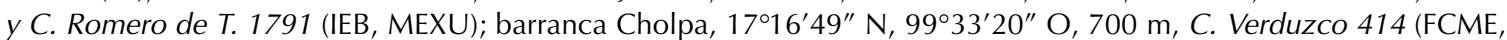
MEXU).

29. Pitcairnia mooreana L.B.Sm., Contr.U.S.Natl.Herb. 29:523-524, f. 79. 1954.

Figura 13

DISTRIBUCIÓN: Endémica de México.

ALTITUD: ca. 960 m.

EJEMPLARES EXAMINADOS: Chilpancingo de los Bravo: km 339-40 between Acahuizotla and Agua de Obispo on highway to Acapulco, $17^{\circ} 20^{\prime} 15^{\prime \prime} \mathrm{N}, 99^{\circ} 28^{\prime} 30^{\prime \prime} \mathrm{W}, 3000 \mathrm{ft}$, H. E. Moore 5117 (US).

30. Pitcairnia oaxacana L.B.Sm., Contr.Gray Herb. 117:25-26, t. 2, f. 16, 17. 1937.

Figura 13

DISTRIBUCIÓN: Endémica de México.

ALTITUD: ca. $730 \mathrm{~m}$.

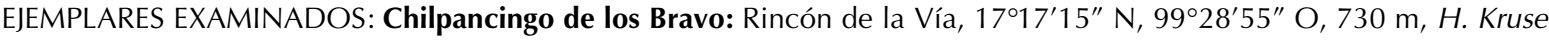
383 (FCME, MEXU, US). José Azueta: Vallecitos, $17^{\circ} 55^{\prime} \mathrm{N}, 101^{\circ} 19^{\prime} \mathrm{O}, \mathrm{G}$. B. Hinton 11366 (GH, LL, MICH, UC, US).

31. Pitcairnia pteropoda L.B.Sm., Contr.Gray Herb. 117:26-27, t. 2, f. 19.1937.

Figura 12 DISTRIBUCIÓN: Endémica de México.

ALTITUD: $1400-1700 \mathrm{~m}$.

EJEMPLARES EXAMINADOS: Coyuca de Catalán: Aguazarca, $18^{\circ} 7^{\prime} \mathrm{N}, 101^{\circ} 00^{\prime} \mathrm{O}$, G. B. Hinton et al. 9450 (GH, US); Tierras Blancas, 189' N, 101 ${ }^{\circ} 7^{\prime}$ O, 1400 m, G. B. Hinton et al. 10141 (MEXU, US). Teloloapan: along Mex. hwy. 51, $17 \mathrm{~km} \mathrm{~W}$ of Teloloapan between $\mathrm{km} 77$ and 78 at quarry. 50 yards above Teloloapan - Arcelia road, $18^{\circ} 25^{\prime} \mathrm{N}, 9^{\circ} 58^{\prime} \mathrm{W}$, $1700 \mathrm{~m}$, H. H. Iltis y T. S. Cochrane 29 (ENCB, MICH, US).

32. Pitcairnia roseana L.B.Sm., Contr.Gray Herb. 117:27-28, t. 2, f. 22, 23. 1937.

Figura 13

DISTRIBUCIÓN: Endémica de México.

ALTITUD: 880-2000 m.

EJEMPLARES EXAMINADOS: Buenavista de Cuéllar: Cañón de la Mano. $1.5 \mathrm{~km}$ al SO de Los Amates, por la vía del FC a El Naranjo, 18²4'56" N, 99²9’50" O, A. García-Mendoza, A. Gutiérrez, S. Franco y A. Castañeda 6733 (MEXU).

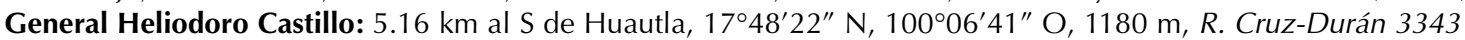

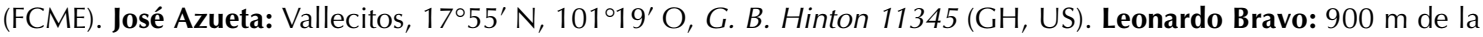

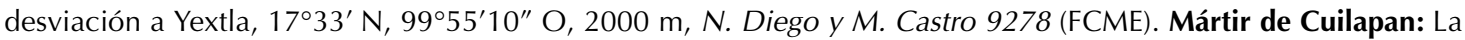
Esperanza, 1745'35" N, 99¹4'58" O, 1600 m, M. J. Sánchez 18 (FCME). Mochitlán: 12 km de Quechultenango por

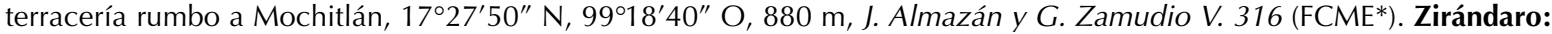
en El Plátano, $12 \mathrm{~km}$ al SE de Guayameo, 18²6 $6^{\prime} \mathrm{N}, 101^{\circ} 3^{\prime} \mathrm{O}, 1250 \mathrm{~m}$, J. C. Soto y G. Silva 4421 (ENCB, MEXU, MO).

33. Pitcairnia sordida L.B.Sm., Contr.Gray Herb. 161:34, t. 4, f. 6.1946.

Figura 12 DISTRIBUCIÓN: Endémica de México.

ALTITUD: $1160-2250 \mathrm{~m}$.

EJEMPLARES EXAMINADOS: Coyuca de Catalán: Carricera, $18^{\circ} 5^{\prime} \mathrm{N}, 101^{\circ} 5^{\prime} \mathrm{O}, 1000-1500 \mathrm{~m}$, G. B. Hinton 10090 (US); I. R. F. del Oro, Trincheras, Vinatita, $18^{\circ} 6^{\prime} \mathrm{N}, 101^{\circ} 16^{\prime} \mathrm{O}$, G. B. Hinton et al. 10127 (GH, MEXU, NY, US). Eduardo Neri:

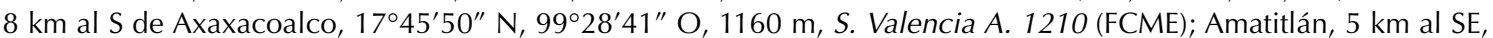

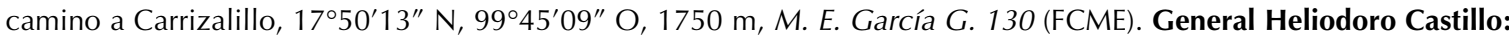

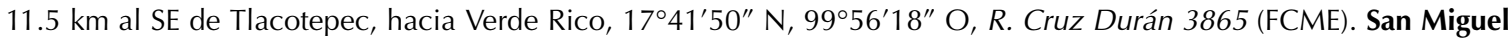
Totolapan: Toro Muerto, $17^{\circ} 34^{\prime} \mathrm{N}, 100^{\circ} 15^{\prime} 30^{\prime \prime} \mathrm{O}, 2250$ m, G. B. Hinton et al. 14248 (ENCB, GH, NY, US).

34. Pitcairnia tillandsioides L.B.Sm., Contr.Gray Herb. 161:35, t. 4, f. 7. 1946.

Figura 13 DISTRIBUCIÓN: Endémica de México.

ALTITUD: $1775 \mathrm{~m}$

EJEMPLARES EXAMINADOS: San Miguel Totolapan: Los Varales "Barrales" - Yesqueros, $17^{\circ} 42^{\prime}$ N, $100^{\circ} 29^{\prime} \mathrm{O}, 1775 \mathrm{~m}$, G. B. Hinton et al. $14398(\mathrm{GH}, \mathrm{US})$.

RACINAEA M. A. SPENCER et L.B.SM

35. Racinaea ghiesbreghtii (Baker) M.A.Spencer et L.B.Sm., Phytologia 74:153. 1993.

Figura 7

DISTRIBUCIÓN: México y Guatemala. 
ALTITUD: $1930 \mathrm{~m}$.

EJEMPLARES EXAMINADOS: Chilapa de Álvarez: ca. 2 km al NNO de El Caracol, camino Atzacoaloya-Hueycaltenango, $17^{\circ} 29^{\prime} \mathrm{N}, 9^{\circ} 4^{\prime} 30^{\prime \prime} \mathrm{O}, 1930 \mathrm{~m}$, F. Lorea 1225 (FCME*).

\section{TILLANDSIA L.}

1. Plantas largamente caulescentes, colgantes

T. usneoides

1. Plantas acaulescentes o muy cortamente caulescentes, erectas a extendidas, pero nunca colgantes.

2. Rosetas pseudobulbosas, las vainas foliares conspicuas, cóncavas, adpresas y a veces algo infladas.

3. Brácteas florales glabras u obscuramente lepidotas.

4. Brácteas florales lisas o nervadas sólo hacia el margen

T. balbisiana

4. Brácteas florales claramente nervadas en toda su superficie

T. caput-medusae

3. Brácteas florales lepidotas a densamente lepidotas.

5. Hojas variegadas con púrpura.

T. butzii

5. Hojas concoloras o manchadas de púrpura sólo en los márgenes.

6. Hojas lisas; las inflorescencias forman keikis y entonces las plantas semejan enredaderas T. intermedia

6. Hojas nervadas a sulcadas; las inflorescencias nunca forman keikis, las plantas nunca semejan enredaderas.

7. Láminas de las hojas largas y recurvadas a enroscadas sobre sí mismas; plantas erectas; inflorescencias compuestas, muy raramente simples.

8. Pseudobulbos globosos; escapo de 5 a $15 \mathrm{~cm}$ de largo; 4 a 6 flores por espiga; sépalos densamente lepidotos

T. pseudobaileyi

8. Pseudobulbos alargados; escapo de 1 a 2 cm de largo; 5-12 flores por espiga; sépalos glabros....... T. lydiae

7. Láminas de las hojas cortas y rígidas, nunca enroscadas sobre sí mismas; plantas falciformes; inflorescencias simples. T. circinnatioides

2. Rosetas nunca pseudobulbosas, las vainas foliares inconspicuas, generalmente planas, extendidas y nunca infladas.

9. Flores polísticas; inflorescencia siempre simple.

10. Sépalos de 25 a $35 \mathrm{~mm}$ de largo; inflorescencia péndula.

11. Vainas de las hojas de color pardo, más oscuras que las láminas y contrastando con ellas.

12. Pétalos azules a morados

T. oaxacana

12. Pétalos verdes a verde amarillentos

T. quaquaflorifera

11. Vainas de las hojas concoloras con las láminas.

13. Sépalos densamente lepidotos, de $35 \mathrm{~mm}$ de largo; pétalos de $6 \mathrm{~cm}$ de largo.

T. macdougallii

13. Sépalos glabros a esparcidamente lepidotos de 10 a $30 \mathrm{~mm}$ de largo; pétalos de 4 a $5 \mathrm{~cm}$ de largo.......

T. andrieuxii

10. Sépalos de 4 a $20 \mathrm{~mm}$ de largo, inflorescencia erecta a extendida.

14. Inflorescencia nidular, el escapo ausente.

15. Inflorescencia simple; rosetas generalmente solitarias; hojas largamente filiformes, de ca. $1 \mathrm{~mm}$ de ancho en la parte media, todas grisáceas. T. magnusiana

15. Inflorescencia compuesta; rosetas generalmente cespitosas; hojas angostamente triangularlanceoladas, de ca. $2 \mathrm{~mm}$ de ancho, las superiores de color rojo. T. ionantha

14. Inflorescencia no nidular, el escapo conspicuo y alargado.

16. Pétalos amarillos; hojas de 5 a $7 \mathrm{~mm}$ de ancho......

T. schiedeana 
16. Pétalos violetas a blancos; hojas de 0.5 a $4 \mathrm{~mm}$ de ancho.

17. Hojas dísticas, de 3 a $10 \mathrm{~cm}$ de largo, recurvadas; inflorescencias con una a dos flores; pétalos de 10 a $12 \mathrm{~mm}$ de largo......

17. Hojas polísticas de 20 a $40 \mathrm{~cm}$ de largo, rectas; inflorescencias capitadas con más de cinco flores; pétalos de $40 \mathrm{~mm}$ de largo.

9. Flores dísticas; inflorescencia compuesta, raramente simple.

18. Vainas de las brácteas primarias igualando o excediendo a sus ramas axilares.

19. Láminas de las hojas subuladas a setáceas

T. pseudosetacea

19. Láminas de las hojas estrecha a largamente triangulares.

20. Láminas de las hojas densamente cinéreo-lepidotas en ambas caras; inflorescencia capitada, pareciendo simple.

T. capitata

20. Láminas de las hojas glabras a glabrescentes, verdes; inflorescencia claramente paniculada.

21. Inflorescencia erecta.

22. Brácteas florales densamente lepidotas

T. bourgaei

22. Brácteas florales glabras.

23. Pétalos verdes; brácteas primarias extendidas, no imbricadas; inflorescencia no estrobiliforme

T. cossonii

23. Pétalos blancos; brácteas primarias adpresas, imbricadas; inflorescencia estrobiliforme.....

T. laui

21. Inflorescencia péndula.

24. Pétalos azules a morados, nunca con tonos verdes o verdosos; brácteas primarias perpendiculares a sus espigas axilares T. violacea

24. Pétalos verdes o verdes con morado; brácteas primarias paralelas a sus espigas axilares.

25. Inflorescencia de cuando mucho $25 \mathrm{~cm}$ de diámetro; brácteas primarias de hasta $15 \mathrm{~cm}$ de largo, con el ápice foliáceo, tan largas o un poco más cortas que sus espigas; pétalos verdes...... T. prodigiosa

25. Inflorescencia de $60 \mathrm{~cm}$ de diámetro; brácteas primarias de hasta $30 \mathrm{~cm}$ de largo, sin láminas, mucho más largas que sus espigas; pétalos verdes con tonos azules o morados. T. mirabilis

18. Vainas de las brácteas primarias más cortas que sus ramas axilares o bien la inflorescencia simple.

26. Espigas laxas, las flores muy separadas unas de otras; brácteas florales cortas, el raquis expuesto.

27. Plantas de ca. $5 \mathrm{~cm}$ de alto; flores 1 a 5 por espiga; láminas de las hojas filiformes.

T. fuchsii

27. Plantas de 10 a $50 \mathrm{~cm}$ de alto; flores muchas más de 5 por espiga; laminas de las hojas triangulares.

28. Estambres exertos; pétalos de color morado claro a blanco, el ápice recto. T. makoyana

28. Estambres incluidos; pétalos de color morado oscuro, el ápice extendido T. gymnobotrya

26. Espigas compactas, las flores muy cercanas unas de otras; brácteas florales imbricadas y cubriendo totalmente al raquis.

29. Inflorescencia con 1 a 3 flores, simple, el escapo corto o ausente.

T. ionantha

29. Inflorescencia con más de 3 flores, simple o compuesta, el escapo evidente.

30. Láminas de las hojas linear-subuladas a filiformes.

31. Brácteas florales membranáceas; rosetas claramente cuadrangulares en la base cuando vivas

T. pentasticha

31. Brácteas florales coriáceas a subcoriáceas; rosetas cilíndricas a redondeadas en la base cuando vivas.

32. Inflorescencias densamente digitadas, algo capitadas. T. juncea 


\section{Valeria Angélica Pulido-Esparza, Ana Rosa López-Ferrari y Adolfo Espejo-Serna}

32. Inflorescencias laxamente pinnadas o simples.

T. ortgiesiana

30. Láminas de las hojas triangulares a estrecha o largamente triangulares.

33. Brácteas florales ecarinadas y sin engrosamiento alguno en la vena media.

34. Vainas de las hojas de color pardo, más oscuras que las láminas y contrastando con ellas.

35. Pétalos azules a morados; brácteas florales no infladas.

36. Estambres exertos; hojas largas, contortas y péndulas.

36. Estambres insertos; hojas cortas y erectas.

T. gymnobotrya

35. Pétalos verdes; brácteas florales infladas.

T. kalmbacheri

34. Vainas de las hojas de color verde, concoloras con las láminas.

37. Inflorescencia compuesta, siempre con tres o más espigas

T. polystachia

37. Inflorescencia simple, raramente con dos espigas.

38. Pétalos verdes; brácteas florales rosadas, conspicuamente nervadas, de 3 a $4 \mathrm{~cm}$ de largo.

T. achyrostachys

38. Pétalos azules a morados; brácteas florales rojas con el margen verde, lisas, de ca. $2.5 \mathrm{~cm}$ de largo

T. guerreroensis

33. Brácteas florales carinadas o al menos con un engrosamiento extra en la vena media.

39. Láminas de las hojas verdes, no notablemente cinéreo o ferrugíneo-lepidotas.

40. Haz de las vainas de las hojas de color pardo, más oscuras que las láminas y contrastando con ellas.

41. Espigas de 30 a $60 \mathrm{~mm}$ de ancho.

42. Pétalos verdes; espigas comprimidas, aplanadas.

T. supermexicana

42. Pétalos azules a morados; espigas gruesas, casi cilíndricas.

43. Espigas de 30 a $40 \mathrm{~cm}$ de ancho; pétalos de $4.5 \mathrm{~cm}$ de largo.

T. langlasseana

43. Espigas de 50 a $60 \mathrm{~mm}$ de ancho; pétalos de $6.5 \mathrm{~cm}$ de largo.

T. trauneri

41. Espigas de 10 a $20 \mathrm{~mm}$ de ancho.

44. Pétalos verdes; espigas de 5 a $6(-8) \mathrm{cm}$ de largo, por 2 a $2.5 \mathrm{~cm}$ de ancho.

T. cossonii

44. Pétalos azules a morados; espigas de (5-)7 a $18 \mathrm{~cm}$ de largo, por 1 a $2 \mathrm{~cm}$ de ancho.

45. Espigas 1 a 3; plantas de menos de $40 \mathrm{~cm}$ de alto, con un rizoma basal conspicuo;

lámina de las hojas de menos de $10 \mathrm{~mm}$ de ancho.

T. tricolor

45. Espigas 5 a 20; plantas de más de $60 \mathrm{~cm}$ de alto, sin rizoma; lámina de las hojas de más de $20 \mathrm{~mm}$ de ancho.

46. Láminas de las brácteas primarias alargadas, filiformes y excediendo por mucho a sus espigas axilares; brácteas florales inconspicuamente lepidotas.....

T. calothyrsus

46. Láminas de las brácteas primarias cortas, las inferiores atenuadas e igualando a sus espigas axilares, las superiores agudas y mucho más cortas que sus espigas axilares; brácteas florales glabras.

47. Inflorescencia bipinnada, espigas de $10-13 \mathrm{~cm}$ de largo

T. paraisoensis

47. Inflorescencia tripinnada, espigas de 5 a $6 \mathrm{~cm}$ de largo.

T. rettigiana

40. Haz de las vainas de las hojas de color verde, concoloras con las láminas.

48. Brácteas primarias escasamente más largas que las brácteas florales o bien la inflorescencia simple; pétalos azules a morados; plantas de la costa, creciendo entre los 0 y los $220 \mathrm{~m} \mathrm{snm}$....... 
48. Brácteas primarias de más de la mitad del largo que sus espigas axilares; inflorescencia siempre compuesta; pétalos verdes; plantas de la sierra, creciendo entre los 1200 y los $1800 \mathrm{~m} \mathrm{snm}$

39. Láminas de las hojas notable y densamente ferrugíneo a cinéreo-lepidotas.

49. Brácteas florales densamente lepidotas.

50. Pétalos verdes; espigas ovaladas a elípticas. T. bourgaei

50. Pétalos blancos, violeta-pálidos inmediatamente arriba de los sépalos; espigas alargadas, oblongas.

49. Brácteas florales glabras a glabrescentes.

51. Láminas de las hojas contortas, péndulas. T. xerographica

51. Láminas de las hojas no contortas, erectas.

52. Inflorescencia simple, raramente subdigitada a subfasciculada.

53. Vainas de las hojas de color verde, concoloras con la lámina.....

T. concolor

53. Vainas de las hojas de color pardo, más oscuras que las láminas y contrastando con ellas.

54. Pétalos azules a morados; plantas de la sierra, creciendo entre los 700 y los 2000 m snm. T. fasciculata

54. Pétalos verdes; plantas de la costa, creciendo entre los 50 y los 300 m snm..... T. marabascoensis

52. Inflorescencia compuesta, paniculada, tirsiforme o fasciculada a subfasciculada.

55. Espigas divergentes a extendidas o recurvadas; inflorescencia laxa a sublaxa.

56. Pétalos verdes.

57. Brácteas florales infladas, glabras, pruinosas; espigas cilíndricas

T. kalmbacheri

57. Brácteas florales adpresas, no infladas, densamente lepidotas; espigas aplanadas.

T. supermexicana

56. Pétalos azules a morados.

58. Inflorescencias paniculadas, con 10 a 14 espigas, éstas engrosadas, casi clíndricas, de 7 a $10 \mathrm{~cm}$ de largo; brácteas florales de 2.5 a $3 \mathrm{~cm}$ de largo..... T. langlasseana

58. Inflorescencias fasciculadas, con 2 a 5 espigas, éstas comprimidas, aplanadas, de 18 a $30 \mathrm{~cm}$ de largo; brácteas florales de 4 a $4.5 \mathrm{~cm}$ de largo. T. fasciculata

55. Espigas estrictas; inflorescencia densa.

59. Vainas de las brácteas primarias inferiores de cerca de la mitad o más del largo que su espiga axilar; espigas de menos de $10 \mathrm{~cm}$ de largo, sésiles a subsésiles. T. bourgaei

59. Vainas de las brácteas primarias inferiores mucho más cortas que sus espigas axilares; espigas de más de $15 \mathrm{~cm}$ de largo, largamente pedunculadas.

T. taxcoensis

36. Tillandsia achyrostachys E.Morren ex Baker, Handb.Bromel. 171. 1889.

Figura 14

DISTRIBUCIÓN: Endémica de México.

ALTITUD: 800-1900 m.

EJEMPLARES EXAMINADOS: Ahuacuotzingo: Ajuatetla, $17^{\circ} 47^{\prime} \mathrm{N}, 99^{\circ} 4^{\prime} 5^{\prime \prime} \mathrm{O}, 1900 \mathrm{~m}$, E. Martínez 46 (FCME). Alpoyeca:

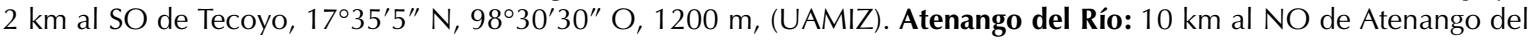

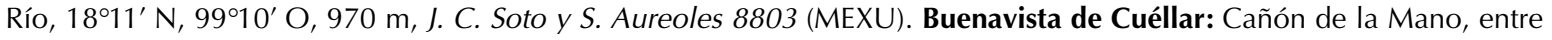
Los Amates y El Naranjo, $10 \mathrm{~km}$ al N de Iguala por el ferrocarril, $18^{\circ} 25^{\prime} \mathrm{N}, 99^{\circ} 30^{\prime} \mathrm{O}, 900-1000 \mathrm{~m}, C$. Catalán, F. Terán y S. Vázquez 549 (ENCB, MEXU); Palmilla, $19 \mathrm{~km}$ al SE de Amacuzac, $18^{\circ} 32^{\prime} \mathrm{N}, 9^{\circ} 25^{\prime} \mathrm{O}, 1100 \mathrm{~m}$, A. R. López-Ferrari 355 (IEB MEXU, UAMIZ). Chilapa de Álvarez: Atzacoaloya, 17³3'50” N, 99²' $5^{\prime \prime}$ O, 910 m, N. Diego 1808 (FCME). Chilpancingo de los Bravo: 3 km al O de Chilpancingo, 17³3’ N, 99³2' O, 1400 m, M. Blanco y C. Toledo 129 
Valeria Angélica Pulido-Esparza, Ana Rosa LóPEZ-Ferrari y Adolfo Espejo-SERna

(ENCB); Cerro de los Bueyes al N de Chilpancingo, 17³4' N, 99²9’30" O, 1330-1500 m, J. Chavelas ES-2876 (ENCB); cerca de Chilpancingo, $17^{\circ} 34^{\prime} \mathrm{N}, 9^{\circ} 30^{\prime} \mathrm{O}, 1200 \mathrm{~m}$, J. Chavelas s. $n$. (MEXU); microondas de Cabañas, por carretera Chilpancingo a Chichihualco, 17³2'58" N, 99³0'42" O, 1450 m, M. Cházaro, B. L. Mostul y F. Madariaga 7573 (IEB, $\mathrm{MICH}, \mathrm{XAL})$; Petaquillas, $30 \mathrm{~km}$ al S de Chilpancingo, carretera Chilpancingo - Acapulco, $17^{\circ} 29^{\prime} 30^{\prime \prime} \mathrm{N}, 99^{\circ} 27^{\prime} \mathrm{O}, 1^{2} 22$ m, M. Flores, A. Martínez, S. Camargo y J. Santana 1008 (UAMIZ); Chilpancingo, carretera a Chichihualco. [colectada en paraje Casa de Tejas al $\mathrm{O}$ en falda $\mathrm{O}$ de barranca con vereda a Amojileca], H. Kruse 3129 (FCME); $3 \mathrm{~km}$ al NO de

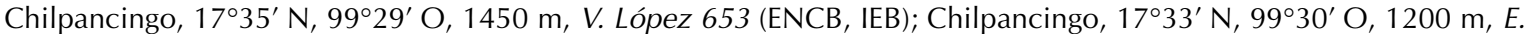
Matuda 38417 (MEXU); en las afueras de Chilpancingo, al NO, $17^{\circ} 34^{\prime} \mathrm{N}, 99^{\circ} 29^{\prime} 30^{\prime \prime} \mathrm{O}, 1300 \mathrm{~m}, \mathrm{C}$. Toledo y M. Blanco 125 (MEXU). Eduardo Neri: $12.3 \mathrm{~km}$ al N de Zumpango del Río, 17²4'30" N, 99³0'00" O, $1500 \mathrm{~m}$, J. Calónico S.

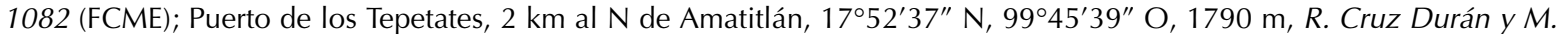

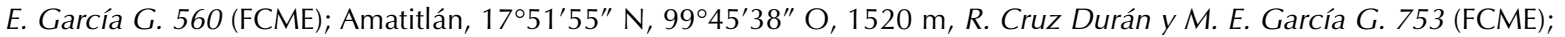
Xochipala, camino a Papalotepec, $17^{\circ} 49^{\prime} \mathrm{N}, 9^{\circ} 38^{\prime} \mathrm{O}$, C. Franco 5 (FCME, MEXU); barranca de Acatitlán, $17^{\circ} 50^{\prime} \mathrm{N}$,

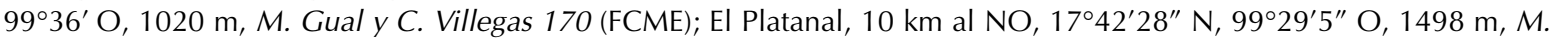
Luna F. 814 (FCME, MEXU); $6 \mathrm{~km}$ al S del río Zumpango, carretera a Chilpancingo, $17^{\circ} 37^{\prime} \mathrm{N}, 99^{\circ} 31^{\prime} \mathrm{O}, 1500 \mathrm{~m}, A . M$. Pascoe 282 (ENCB, IEB); carretera Filo de Caballo - Chichihualco - Chilpancingo, $12 \mathrm{~km}$ antes del entronque México Chilpancingo, 17³7'28" N, 99³4'58" O, 1550 m, A. C. Sánchez 288 (FCME*); 28 km al S de Milpillas, sobre la carretera Iguala - Chilpancingo, 17³2' N, 99³0' O, 900 m, J. C. Soto y S. Aureoles 8892 (MEXU); 5.7 km al O de Chilpancingo ó $2 \mathrm{~km}$ al E de Jalapa, hacia Chichihualco, $17^{\circ} 37^{\prime} \mathrm{N}, 99^{\circ} 34^{\prime} \mathrm{O}$, R. Torres, M. P. Ramírez de A. y T. P. Ramamoorthy 7685

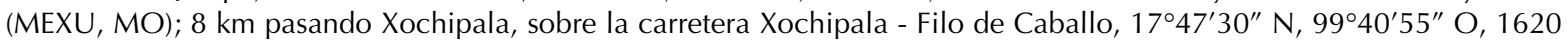

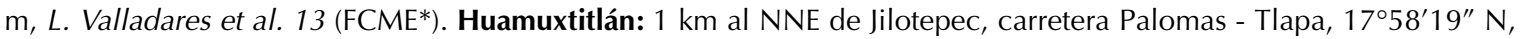
98²8'44" O, 1260 m, E. Moreno G. y M. A. Monroy 748 (FCME), 749 (FCME). Huitzuco de los Figueroa: en Amatitlán, $12 \mathrm{~km}$ al SE de Huitzuco, 18 $15^{\prime} \mathrm{N}, 99^{\circ} 14^{\prime} 30^{\prime \prime} \mathrm{O}, 1100 \mathrm{~m}$, J. C. Soto y S. Aureoles 8773 (MEXU). José Azueta: en San

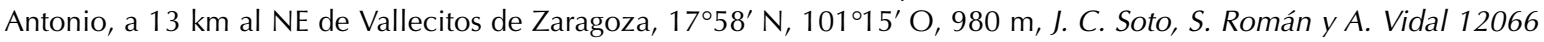

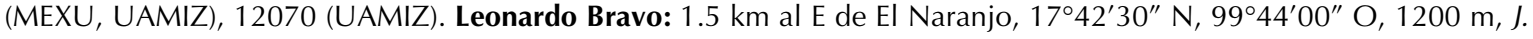
Calónico S. 6940 (FCME); 10.4 km al SO de Chichihualco, 17³6’48" N, 9945’41" O, 1350 m, J. Calónico S. 8335 (FCME); 4 km adelante de Atlixtac por la carretera Chilpancingo - Chichihualco, $1460 \mathrm{~m}, 17^{\circ} 38^{\prime} 30^{\prime \prime} \mathrm{N}, 99^{\circ} 39^{\prime} \mathrm{O}, 1460$ m, F. Lorea, R. M. Fonseca J. y R. Vilchis C. s. n. (FCME). Pilcaya: 21 km al SE de La Concepción, 18 $44^{\prime} 11^{\prime \prime} \mathrm{N}$, 9940'51" O, 1380 m, J. Calónico S. 6042 (FCME*); 3 km al O de Cacahuamilpa, 1841'01" N, 99³1'47" O, 1490 m, J. Calónico S. 8583 (FCME*); 9 km al E de Piedras Negras, 1841'04" N, 99³1'41" O, 1500 m, R. Cruz-Durán 2452 (FCME*). Taxco de Alarcón: $1.5 \mathrm{~km}$ al SE de Tehuilotepec, 18³2'16" N, 99³2'14" O, $1550 \mathrm{~m}$, J. Calónico 4710 (FCME*); 14 km al S de Casahuatlán, 18³3'54" N, 99²8'8" O, 1520 m, J. Calónico 7013 (FCME, MEXU); 8 km al O de Puente Campuzano, $18^{\circ} 26^{\prime} 4^{\prime \prime} \mathrm{N}, 9^{\circ} 39^{\prime} 37^{\prime \prime} \mathrm{O}, 1200 \mathrm{~m}$, J. Calónico 8050 (FCME); $15.3 \mathrm{~km}$ al E de Taxco, 18³3'46" N, 99²8'13" O, 1500 m, J. Calónico 9311 (FCME*); ca. 0.5 km después de Mexcaltepec y 4 km adelante de El Naranjo, carretera Iguala - Taxco, $18^{\circ} 26^{\prime} \mathrm{N}, 9^{\circ} 33^{\prime} \mathrm{O}, 1010 \mathrm{~m}$, A. R. López-Ferrari y A. Espejo 1757 (CICY, UAMIZ); ca. 2 km sobre la brecha a Las Granadas, a partir de San Francisco Acuitlapan, 18³5' N, 99 32' O, $1530 \mathrm{~m}$, A. R. López-Ferrari y A. Espejo 1761 (UAMIZ). Teloloapan: cerca de Teloloapan, carretera Ciudad Altamirano - Iguala, $18^{\circ} 22^{\prime} \mathrm{N}, 99^{\circ} 51^{\prime} \mathrm{O}$, 1600 m, J. C. Soto y S. Aureoles 8209 (MEXU). Tetipac: Tezoapa, entre Chontalcuatlán y Tetipac, 18³8'55" N, 99³5'20" O, 1110 m, A. Espejo, A. R. López-Ferrari, J. Ceja, A. Mendoza e I. Ramírez 6161 (UAMIZ). Tixtla de

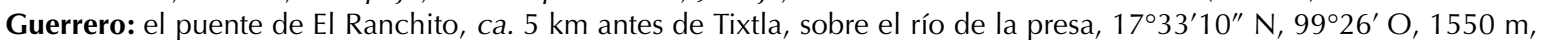
M. Huerta 48 (FCME, UAMIZ); ladera $\mathrm{N}$ del cerro Xomislo, ca. $10 \mathrm{~km}$ por el camino que va de Tixtla a Metlalapa, $17^{\circ} 35^{\prime} 21^{\prime \prime} \mathrm{N}, 9^{\circ} 25^{\prime} 40^{\prime \prime} \mathrm{O}, 1650$ m, E. Domínguez-Licona 310 (FCME, UAMIZ); presa El Molino, ladera poniente, ca.

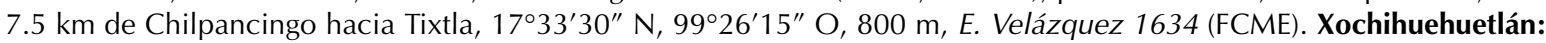

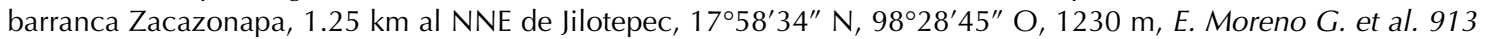
(FCME). Zitlala: Topiltepec, 17³9'1" N, 99¹3'2" O, 1500 m, N. Diego y B. González H. 7298 (FCME).

37. Tillandsia andrieuxii (Mez) L.B.Sm., Contr.Gray Herb. 117:31. 1937.

Figura 14 DISTRIBUCIÓN: Endémica de México.

ALTITUD: $2360-2380 \mathrm{~m}$.

EJEMPLARES EXAMINADOS: Pedro Ascencio Alquisiras: $1.9 \mathrm{~km}$ al NE de Puerto Oscuro, $18^{\circ} 37^{\prime} 23^{\prime \prime} \mathrm{N}, 9^{\circ} 46^{\prime} 41^{\prime \prime} \mathrm{O}$, $2380 \mathrm{~m}, R$, Cruz-Durán 2582 (FCME*). Tetipac: 19.9 km al NO de Taxco, 18³8'03"N , 9945'23" O, $2360 \mathrm{~m}, R$, CruzDurán 2270 (FCME*).

38. Tillandsia balbisiana Schult. et Schult.f., Syst.Veg. 7:1212. 1830.

Figura 14

DISTRIBUCIÓN: Estados Unidos, México, Las Antillas, Colombia y Venezuela.

ALTITUD: 0-116 m.

EJEMPLARES EXAMINADOS: Acapulco de Juárez: El Quemado, orilla de la Laguna de Tres Palos, 16 $45^{\prime} 30^{\prime \prime} \mathrm{N}$, 99³9'55" O, 10 m, N. Diego 4788 (FCME); La Venta, 1654'30" N, 9948' O, 50 m, F. A. Villanueva s. n. (IEB). La 
FLora BROMELIOLÓGICA DE GUERRERO

Unión de Isidoro Montes de Oca: ca. $14 \mathrm{~km}$ al $\mathrm{N}$ de Coyuquilla, rumbo a Infiernillo, por la carretera de cuota Lázaro

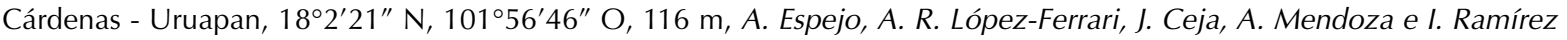
6600 (UAMIZ). Petatlán: 4 km La Salada - El Zarco, 17³2'45" N, 101²5' O, N. Diego 2420 (FCME).

OTROS REGISTROS: Acapulco de Juárez: Acapulco, 1652’ N, 9954’ O, 0 m, Hancock 51 (K) [Smith y Downs, 1977: 986].

39. Tillandsia bourgaei Baker, J.Bot. 25:278. 1887.

Figura 14

DISTRIBUCIÓN: Endémica de México.

ALTITUD: 500-3100 m

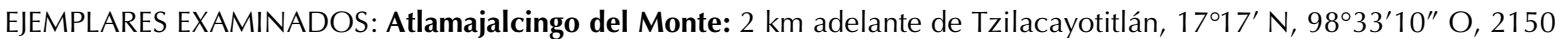

$\mathrm{m}$, F. Lorea y A. Palacios s. n. (FCME). Chilpancingo de los Bravo: km 19 carretera Chilpancingo - Omiltemi

"Omiltepec", 1733' N, 99³8' O, Bradovsky s. n. (MEXU); km 26 carretera Chilpancingo - Omiltemi "Omiltepec", $17^{\circ} 33^{\prime} \mathrm{N}, 9^{\circ} 39^{\prime} \mathrm{O}$, Bradovsky s. n. (MEXU); cerca de Chilpancingo, $17^{\circ} 34^{\prime} \mathrm{N}, 99^{\circ} 30^{\prime} \mathrm{O}, 1200 \mathrm{~m}$, J. Chavelas s. $n$.

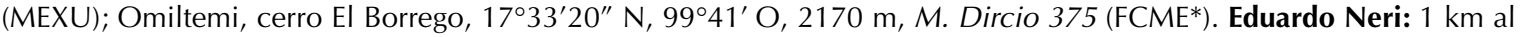

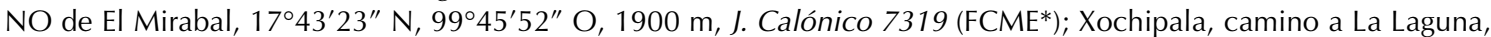
$17^{\circ} 47^{\prime} \mathrm{N}, 99^{\circ} 42^{\prime} \mathrm{O}, 1500 \mathrm{~m}$, C. Franco 27 (MEXU), s. n. (MEXU). General Heliodoro Castillo: $10 \mathrm{~km}$ al NO de Filo de

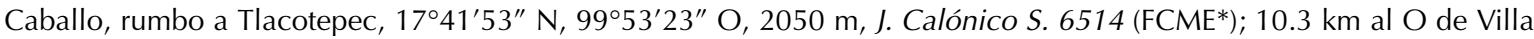
Xóchil, $17^{\circ} 41^{\prime} 16^{\prime \prime} \mathrm{N}, 100^{\circ} 05^{\prime} 07^{\prime \prime} \mathrm{O}, 1850 \mathrm{~m}$, J. Calónico S.13203 (FCME); $8 \mathrm{~km}$ al SO de puerto El Jilguero, camino a Puerto del Gallo, $17^{\circ} 28^{\prime} \mathrm{N}, 100^{\circ} 2^{\prime} 34^{\prime \prime}$ O, F. Lorea 3937 (FCME, UAMIZ); Cerro Teotepec, $17^{\circ} 28^{\prime}$ N, $100^{\circ} 8^{\prime} \mathrm{O}, 3100$ m,

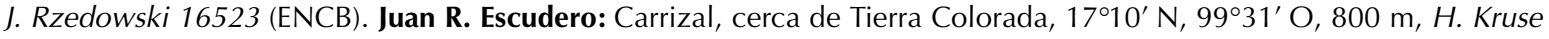
3125 sub E. Matuda 38658 (MEXU); Tierra Colorada, 179'56" N, 99³1'35" O, 500 m, E. Matuda 38673 (MEXU).

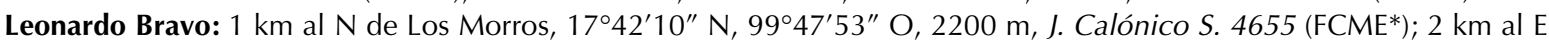
de Campo de Aviación, 17³9'55" N, 9948’34" O, 2150 m, J. Calónico S. 8037 (FCME*); Filo de Caballo, 17³9' N, 9951' O, 2100 m, Romero Domínguez s. n. (FCME), J. C. Soto y S. Aureoles 8282 (MEXU). Mochitlán: Ximilcotitlán

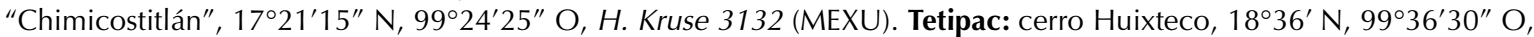
2200 m, N. Diego, M. Gispert y A. Campos 7814 (FCME). Tixtla de Guerrero: ladera N del cerro Xomislo, ca. $10 \mathrm{~km}$ por el camino que va de Tixtla a Metlalapa, $17^{\circ} 35^{\prime} 21^{\prime \prime} \mathrm{N}, 99^{\circ} 25^{\prime} 40^{\prime \prime} \mathrm{O}, 1800 \mathrm{~m}$, E. Domínguez-Licona 323 (FCME). Zitlala:

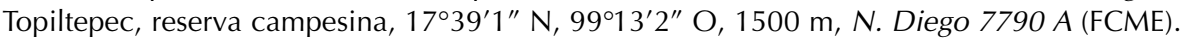

40. Tillandsia butzii Mez, in Engl., Pflanzenr. IV. 32 (Heft 100, 4): 636. 1935.

Figura 14

DISTRIBUCIÓN: México a Panamá.

ALTITUD: $1880-2100 \mathrm{~m}$.

EJEMPLARES EXAMINADOS: Chilapa de Álvarez: $25 \mathrm{~km}$ al SE de Chilapa, paraje Tlacomulco, $17^{\circ} 30^{\prime} 20^{\prime \prime} \mathrm{N}^{\prime} 9^{\circ} 9^{\circ} 5^{\prime} \mathrm{O}$, 1900 m, S. Acosta y R. López 53 (ENCB, IEB, MEXU); ca. 2 km al NNO de El Caracol, camino Atzacoaloya -

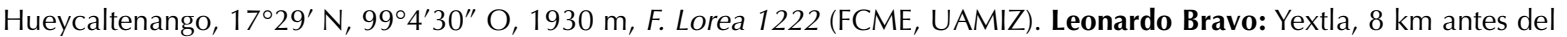

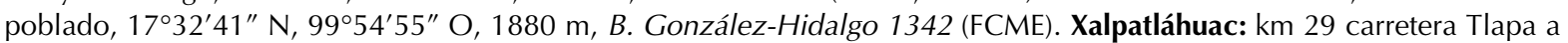
Malinaltepec, $17^{\circ} 23^{\prime}$ N, 98 $93^{\circ} 30^{\prime \prime}$ O, 2100 m, R. Fragoso y J. Santana 1487 (FCME*, UAMIZ).

41. Tillandsia calothyrsus Mez, in C.DC., Monogr.Phan. 9:704-705. 1896.

Figura 15

DISTRIBUCIÓN: Endémica de México.

ALTITUD: 0-2400 m.

EJEMPLARES EXAMINADOS: Chilpancingo de los Bravo: Omiltemi, camino a la Cueva del Tigre, $17^{\circ} 33^{\prime} 35^{\prime \prime} \mathrm{N}$,

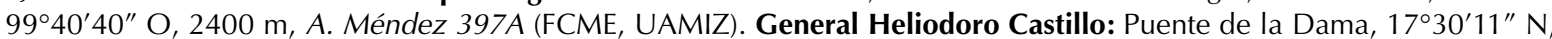
9959'04" O, 1700 m, N. Diego, E. Acosta y G. Fernández 8846 (FCME*). Xalpatláhuac: km 29 carretera Tlapa a Malinaltepec, $17^{\circ} 23^{\prime} \mathrm{N}, 9^{\circ} 34^{\prime} 30^{\prime \prime} \mathrm{O}, 2100 \mathrm{~m}$, R. Fragoso y J. Santana 1491 (FCME*).

42. Tillandsia capitata Griseb., Cat.PI.Cub. 255. 1866.

Figura 15

DISTRIBUCIÓN: México y Las Antillas.

ALTITUD: 0-1080 m.

EJEMPLARES EXAMINADOS: Acapulco de Juárez: east of Acapulco, 16 ${ }^{\circ} 52^{\prime} \mathrm{N}, 9^{\circ} 54^{\prime} \mathrm{W}, 0$ m, R. G. Atkinson 26 (GH).

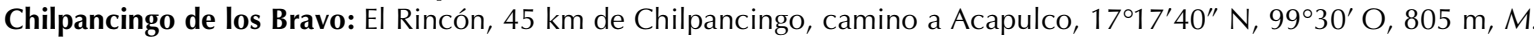
Flores, A. Martínez, S. Camargo y J. Santana 1003 (UAMIZ); Chilpancingo, 17³3' N, 99³0' O, M. B. y R. Foster 1165 (GH); Rincón de la Vía, 17¹7'15" N, 99²8'55" O, 800 m, H. Kruse 1880 (MEXU), 3107 (ENCB, MEXU), H. Kruse 3106 sub Matuda 38630 (MEXU); about 1 mile from Petaquillas on road to Mochitlán and Colotlipa, $17^{\circ} 29^{\prime}$ N, $9^{\circ} 28^{\prime} \mathrm{W}^{\prime} \mathrm{H}$.

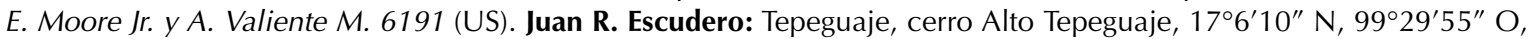
580 m, H. Kruse 2613 (FCME), 3106 (FCME, MEXU). Pilcaya: Cacahuamilpa, 1840’ N, 99॰30’ O, C. Beutelspacher sub E. Matuda s. n. (MEXU). Tlapa de Comonfort: 4 km al N de Tlapa, sobre la carretera Tlapa - Alpoyeca, $17^{\circ} 34^{\prime} \mathrm{N}, 9^{\circ} 33^{\prime}$ O, $1080 \mathrm{~m}$, M. Flores 934 (UAMIZ). 
Valeria Angélica Pulido-Esparza, Ana Rosa LóPeZ-Ferrari y Adolfo Espejo-Serna

OTROS REGISTROS: Chilpancingo de los Bravo: Chilpancingo, $17^{\circ} 33^{\prime} \mathrm{N}, 99^{\circ} 30^{\prime} \mathrm{O}$, O. M. Clark 7218 (MO) [Smith y Downs, 1977: 1003]; G. Webster, C. M. Rowell y F. A. Barkley 17-M-847 (F) [Smith y Downs, 1977: 1003].

43. Tillandsia caput-medusae E.Morren, Belgique Hort. 30: 90. 1880.

Figura 15 DISTRIBUCIÓN: México a Nicaragua.

ALTITUD: $260-2300 \mathrm{~m}$.

EJEMPLARES EXAMINADOS: Acapulco de Juárez: Providencia, $17^{\circ} 6^{\prime} \mathrm{N}, 99^{\circ} 47^{\prime} \mathrm{O}, 900 \mathrm{~m}$, E. Langlassé 1013 bis (P).

Alpoyeca: crucero Tecoyame de Guadalupe - Tecoyo, 17³6' $5^{\prime \prime} \mathrm{N}, 98^{\circ} 29^{\prime} 29^{\prime \prime} \mathrm{O}, 1100$ m, J. Calónico S. 752 (FCME); 1.5 $\mathrm{km}$ al N de Tecoyo, 17³6'10" N, 98²9'29" O, 1200 m, J. Calónico S. 1819 (FCME). Chilpancingo de los Bravo: cerros

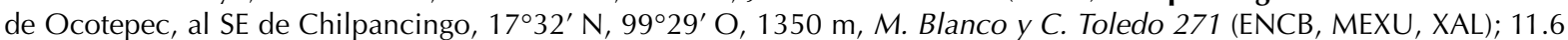

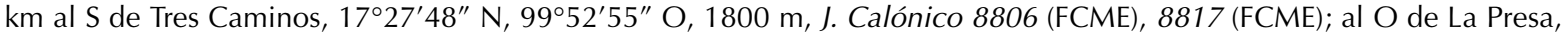

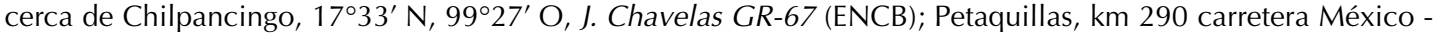

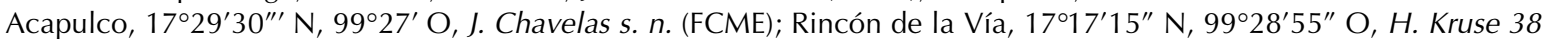

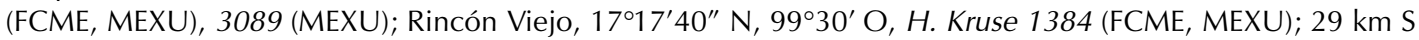
Chilpancingo, $17^{\circ} 19^{\prime} \mathrm{N}, 9^{\circ} 29^{\prime} \mathrm{O}$, B. Leuenberger y C. Schiers 2729 (MEXU); at km 338, $3 \mathrm{~km}$ beyond Acahuizotla on highway to Acapulco, $17^{\circ} 22^{\prime} \mathrm{N}, 9^{\circ} 27^{\prime} \mathrm{W}, \mathrm{H}$. E. Moore y C. E. Wood 4687 (AA, MICH, US); en Jaleaca de Catalán, $17^{\circ} 27^{\prime} \mathrm{N}, 99^{\circ} 51^{\prime} \mathrm{O}, 790 \mathrm{~m}$, J. C. Soto y F. Solórzano 12766 (IBUG,MEXU); $1 \mathrm{~km}$ al NO de El Rincón de la Vía, 17017'46" N, 99²9'16" O, 760 m, C. Verduzco M. 58 (FCME). Coyuca de Catalán: 8 km al SO de El Cundancito, $17^{\circ} 57^{\prime} \mathrm{N}, 101^{\circ} 10^{\prime} 10^{\prime \prime} \mathrm{O}, 1100 \mathrm{~m}$, J. C. Soto, S. Aureoles, G. Silva, C. Pizarras y S. Conejo 8638 (MEXU). Eduardo Neri:

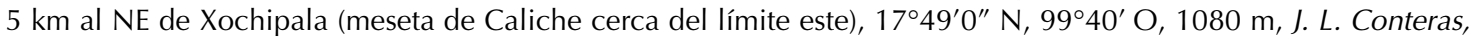
Almanza y Saldívar 212 (FCME*); Amatitlán, 1751'55" N, 9945'38" O, 1520 m, R. Cruz-Durán y M. E. García 754

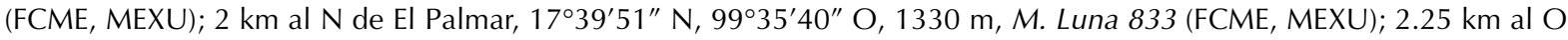

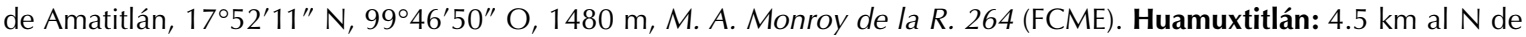
Huamuxtitlán, $17^{\circ} 50^{\prime} \mathrm{N}, 98^{\circ} 34^{\prime} \mathrm{O}$, F. Limón 34 II (FCME); ca. 9 km al N de la desviación a Zapotitlán Lagunas, sobre la

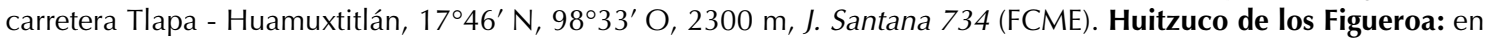

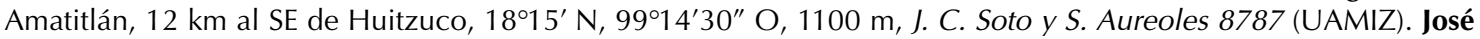
Azueta: $1.5 \mathrm{~km}$ al $\mathrm{SO}$ del caserío La Vainilla, en los cerros al $\mathrm{N}$ del mirador, $17^{\circ} 42^{\prime} \mathrm{N}, 101^{\circ} 31^{\prime} 30 \mathrm{O}, 500 \mathrm{~m}, C$. Gallardo, F. Lorea y G. Ávila 478 (FCME, MEXU); $1.5 \mathrm{~km}$ al NO de el poblado El Sandial, Costa Grande, $17^{\circ} 47^{\prime} 50^{\prime \prime} \mathrm{N}$, 10137'35" O, 260 m, S. Peralta 495 (FCME); 15 km al S de El Viejo, carretera Ciudad Altamirano - Zihuatanejo, 1758' $\mathrm{N}, 101^{\circ} 16^{\prime} \mathrm{O}, 920 \mathrm{~m}, \mathrm{R}$. Torres, P. Tenorio y C. Romero de T. 1284 (MEXU). La Unión de Isidoro Montes de Oca: en El

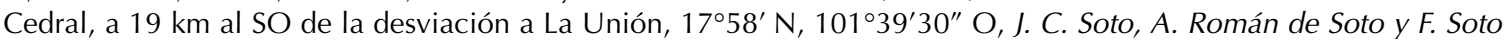

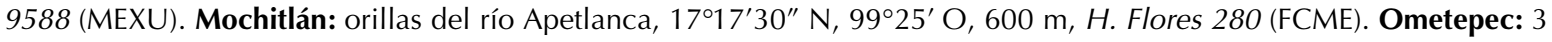
km de Ometepec por terracería, rumbo a Tlacochistlahuaca, 164 $41^{\prime} 35^{\prime \prime}$ N, 98 23'20" O, 350 m, G. Gaxiola 292

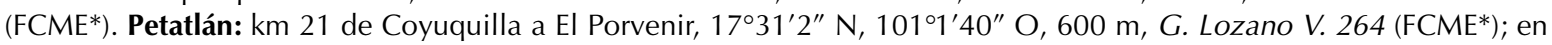

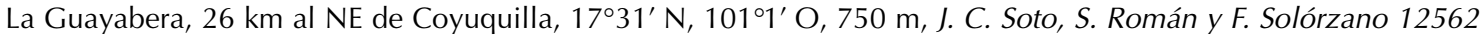

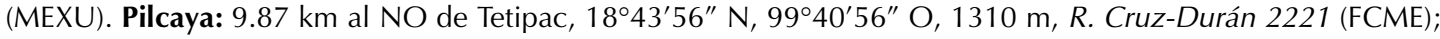
Cacahuamilpa, $18^{\circ} 40^{\prime} \mathrm{N}, 99^{\circ} 30^{\prime} \mathrm{O}$, O. Holguín s. n. (ENCB). Taxco de Alarcón: Taxco Viejo, $18^{\circ} 29^{\prime} \mathrm{N}, 9^{\circ} 35^{\prime} \mathrm{O}, R$. $Q$. Abbott $130(\mathrm{GH})$; ca. 2 km sobre la brecha a Las Granadas, a partir de San Francisco Acuitlapan, $18^{\circ} 35^{\prime} \mathrm{N}, 9^{\circ} 32^{\prime} \mathrm{O}$, $1530 \mathrm{~m}$, A. R. López-Ferrari y A. Espejo 1762 (CICY, UAMIZ); 0.5 km al N de Puente Campuzano, carretera Iguala Taxco, $18^{\circ} 27^{\prime} \mathrm{N}, 99^{\circ} 35^{\prime} 20^{\prime \prime} \mathrm{O}$, M. Martínez G.1567 (FCME). Tetipac: La Piedra China, $3 \mathrm{~km}$ sobre la brecha a Chontalcuatlán, 18³7'43" N, 99³3'12" O, 1350 m, A. Espejo, A. R. López-Ferrari, J. Ceja, A. Mendoza e I. Ramírez

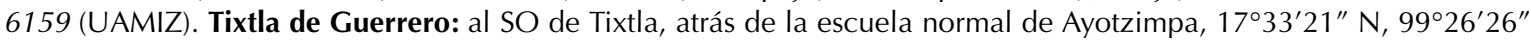
$\mathrm{O}, 1500 \mathrm{~m}$, R. M. Fonseca 2332 (FCME). Tlalixtaquilla de Maldonado: $1 \mathrm{~km}$ al $\mathrm{N}$ de Tecoyo, barranca Los Guayabos, $17^{\circ} 35^{\prime} 45^{\prime \prime} \mathrm{N}, 98^{\circ} 28^{\prime} 28^{\prime \prime} \mathrm{O}, 1050 \mathrm{~m}$, J. Calónico S. 1795 (FCME). Tlapa de Comonfort: $4 \mathrm{~km}$ al N de Tlapa, sobre la carretera Tlapa - Alpoyeca, 17³4' N, 98³3' O, 1080 m, M. Flores, A. Martínez, J. Santana y S. Camargo 933 (UAMIZ); cerro cerca de Los Otates, ca. 15 km al E de Tlapa, 17³4’ N, 98³1’ O, 910 m, A. García-Mendoza, S. Franco y A. Castañeda 6878 (MEXU). Xalpatláhuac: alrededores de San Nicolás Zoyatlán, por la carretera Tlapa - Chilapa, al SO de Tlapa, $17^{\circ} 28^{\prime} \mathrm{N}, 98^{\circ} 39^{\prime} \mathrm{O}, 2300$ m, J. Santana, M. Pérez y C. Toledo 402 (UAMIZ). Xochihuehuetlán: cañada Las Pozas,

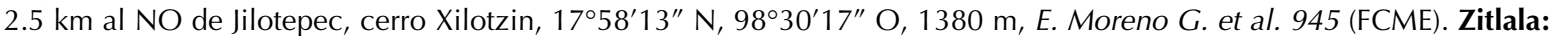
Topiltepec, 17³9'1" N, 99¹3'12" O, 1500 m, N. Diego y B. González 7299 (FCME), 7302 (FCME).

OTROS REGISTROS: José Azueta: Vallecitos, $17^{\circ} 55^{\prime} \mathrm{N}, 101^{\circ} 19^{\prime} \mathrm{O}, \mathrm{G}$. B. Hinton 10227 (K) [Smith y Downs, 1977: 988].

44. Tillandsia circinnatioides Matuda, Cact.Succ.J. (Los Angeles) 45: 187, f. 4, 4a, 5. 1973.

Figura 15 DISTRIBUCIÓN: Endémica de México.

ALTITUD: 700-1500 m.

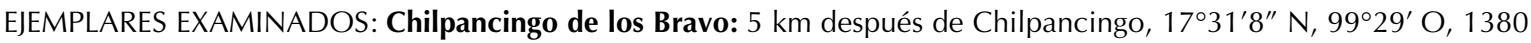

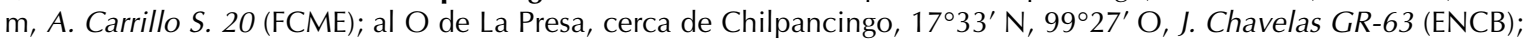


FLORA BROMELIOLÓGICA DE GUERRERO

km 4.5 a Jalapa, $17^{\circ} 35^{\prime} 30^{\prime \prime} \mathrm{N}, 9^{\circ} 31^{\prime} 5^{\prime \prime} \mathrm{O}, \mathrm{H}$. Kruse 2516 (FCME, MEXU); en una barranca seca cerca de Chilpancingo,

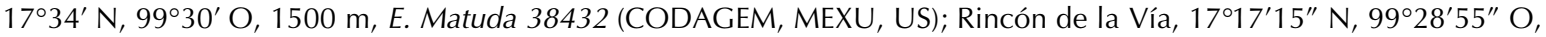

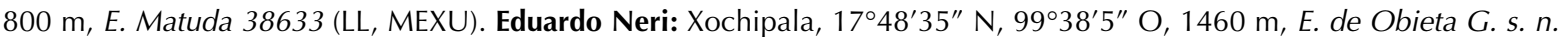
(FCME); puerto El Rancho, ladera O del cruce de Tres Caminos (rumbo Ameyaltepec), $17^{\circ} 57^{\prime} \mathrm{N}, 99^{\circ} 32^{\prime} \mathrm{O}, 700 \mathrm{~m}, E$.

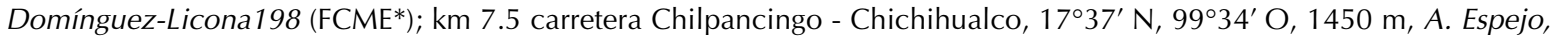
A. Flores y V. Sosa 4638 (UAMIZ); 6 km sobre la desviación a Xochipala, carretera Iguala - Chilpancingo, 17²48’ N, $99^{\circ} 37^{\prime}$ O, M. Flores, A. Martínez, S. Camargo y J. Santana 993 (UAMIZ); Xochipala, camino a las ruinas, $17^{\circ} 49^{\prime} \mathrm{N}$,

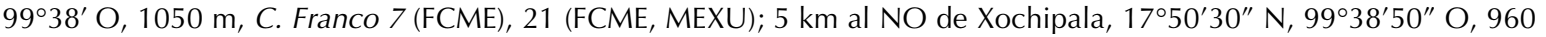
m, M. Gual D. y C. Villegas 109 (FCME); Jalapa, en el km 13.5 de la carretera a Chichihualco, $17^{\circ} 37^{\prime} 40^{\prime \prime}$ N, 99 $9^{\circ} 35^{\prime} 40^{\prime \prime}$

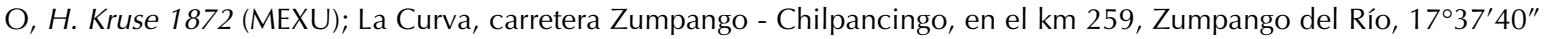
N, 99³2'30" O, H. Kruse 3115 (FCME, MEXU); 85397 hm (sic) sobre la carretera Chilpancingo - Filo de Caballo

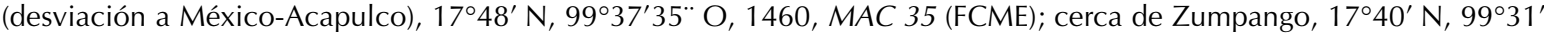
O, 900 m, E. Matuda 38291 (MEXU, US); in Cañón del Zopilote, just $\mathrm{N}$ of Venta Vieja, km 263 on highway between

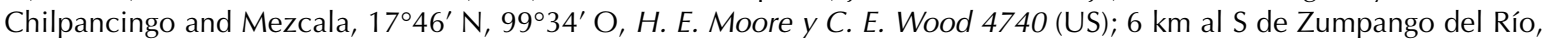
sobre la carretera a Chilpancingo, $17^{\circ} 37^{\prime} \mathrm{N}, 99^{\circ} 31^{\prime} \mathrm{O}, 1500 \mathrm{~m}$, A. M. Pascoe 283 (ENCB); cerca de la desviación a Xochipala, 1747'30" N, 99³3'35" O, 1015 m, S. Peralta G. y C. Villegas 138 (FCME); Cañón del Zopilote, $10 \mathrm{~km}$ al N de Zumpango, $17^{\circ} 45^{\prime} \mathrm{N}, 99^{\circ} 34^{\prime} \mathrm{O}, 800 \mathrm{~m}$, J. Rzedowski 16538 (ENCB); $9 \mathrm{~km}$ por la desviación a Filo de Caballo (1 km antes de Xochipala), a partir del camino de Iguala a Chilpancingo, más o menos $42 \mathrm{~km}$ al NO de Chilpancingo, $17^{\circ} 49^{\prime}$ N, 99³9' O, 1095 m, J. Santana, A. Martínez y S. Camargo 368 B (IEB, UAMIZ). Huamuxtitlán: 0.6 km al NO de

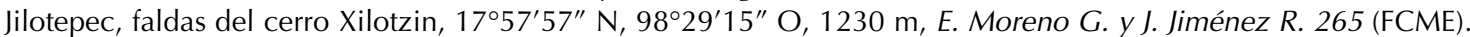
Tepecoacuilco de Trujano: carretera Iguala - Chilpancingo, $2 \mathrm{~km}$ al $\mathrm{O}$ de la desviación a Coacoyula, $18^{\circ} 5^{\prime} 27^{\prime \prime} \mathrm{N}$, 99³3'58" O, 860 m, J. C. Soto y S. Aureoles 8836 (MEXU). Xochihuehuetlán: 5.7 km después de Xochihuhuetlán, sobre

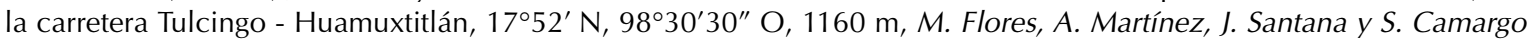
931 (UAMIZ); barranca Zacazonapa, $1.25 \mathrm{~km}$ al NNE de Jilotepec, E. Moreno G. et al. 911 (FCME).

45. Tillandsia concolor L.B.Sm., Phytologia 7:249, t. 1, f. 1-2. 1960.

Figura 15 DISTRIBUCIÓN: México y El Salvador.

ALTITUD: ca. $80 \mathrm{~m}$.

EJEMPLARES EXAMINADOS: Coahuayutla de José María Izazaga: a 14 km de La Unión, hacia Coahuayutla, $18^{\circ} 4^{\prime} 15^{\prime \prime} \mathrm{N}$, 10143' O, 80 m, G. Campos R. 294b (FCME*, UAMIZ).

46. Tillandsia cossonii Baker, J.Bot. 25:279. 1887.

Figura 16

DISTRIBUCIÓN: Endémica de México.

ALTITUD: $1720-2600 \mathrm{~m}$.

EJEMPLARES EXAMINADOS: Atoyac de Álvarez: ca. 3 km de Puerto del Gallo en dirección NE, ladera O del cerro Teotepec, $17^{\circ} 27^{\prime} 40^{\prime \prime} \mathrm{N}, 100^{\circ} 9^{\prime} 30^{\prime \prime}$ O, 2430 m, E. Domínguez-Licona 1299 (FCME); ca. 3 km antes de El Gallo, frente a la entrada a Las Pozas, O del Teotepec, $17^{\circ} 27^{\prime} 50^{\prime \prime} \mathrm{N}, 100^{\circ} 10^{\prime} \mathrm{O}, 2550 \mathrm{~m}$, E. Velázquez M. 2036 (FCME). Chilpancingo

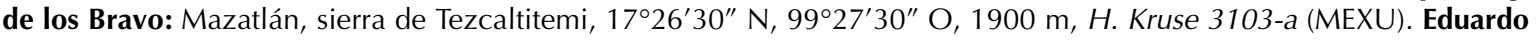
Neri: 12 km adelante de Xochipala, rumbo a Filo de Caballo, 1746'40" N, 9942" O, 1720 m, A. R. López-Ferrari 361 (UAMIZ). Leonardo Bravo: 4 km al S de Carrizal de Bravos, 17³5'07" N, 9950'49" O, 2600 m, J. Calónico S. 8319

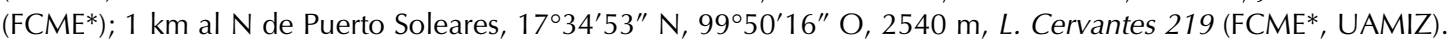

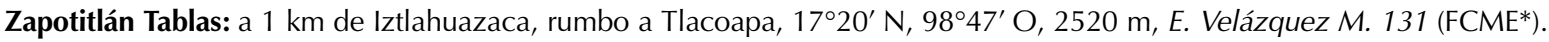

47. Tillandsia fasciculata Sw., Prodr. 56. 1788.

DISTRIBUCIÓN: Estados Unidos, México, Las Antillas, Centroamérica y norte de Sudamérica.

Figura 16

ALTITUD: 720-1900 m.

EJEMPLARES EXAMINADOS: Atenango del Río: 5 km al N de Atenango del Río, rumbo a Comala, 18 $8^{\circ} 8^{\prime} 2^{\prime \prime} \mathrm{N}^{\prime} 9^{\circ} 9^{\circ} 10^{\prime \prime}$ O, 720 m, J. L. Contreras J. 2428 (FCME). Atlixtac: ca. 4 km después de Atlixtac, rumbo a Petatlán, 17³4'41" N, 98 59'31" O, 1500 m, L. Mendizábal B. 56 (FCME*); ca. 2 km después de Atlixtac, rumbo a Petatlán, 17³4'36" N,

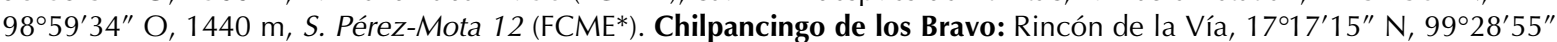

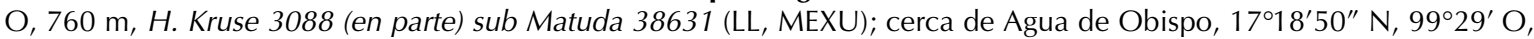
$1500 \mathrm{~m}$, E. Matuda 38684 (MEXU); at km 338 on road to Acapulco, about $3 \mathrm{~km}$ beyond Acahuizotla, $17^{\circ} 22^{\prime} \mathrm{N}^{\prime}, 9^{\circ} 27^{\prime}$ W, ca. 960 m, H. E. Moore y A. Valiente 6195 (US); 4 miles W of Chilpancingo, 17³3' N, 99³0' O, C. M. Rowell y Hamilton 3357 (MICH). Eduardo Neri: barranca de Acatitlán, $17^{\circ} 50^{\prime} \mathrm{N}, 99^{\circ} 36^{\prime} \mathrm{O}, 1020 \mathrm{~m}, \mathrm{M}$. Gual y C. Villegas 170 (FCME). General Heliodoro Castillo: 4 km al NE de Yextla, 17³8'24" N, 9955'43" O, 1450 m, J. Calónico S. 8246 (FCME*); en Sierra de Yerbabuena, Sierra de Guerrero, $17^{\circ} 31^{\prime} \mathrm{N}, 9^{\circ} 58^{\prime} \mathrm{O}, 1900 \mathrm{~m}$, E. Matuda 38650 (MEXU). Ixcateopan de Cuauhtémoc: $5 \mathrm{~km}$ al S de Ixcateopan de Cuauhtémoc, 18²9’ N, 9947’ O, 1600 m, J. Calónico 2665 
(FCME*). Leonardo Bravo: 7.2 km al SE de Filo de Caballo, 1740'39" N, 9946'38" O, 1350 m, J. Calónico S. 9013

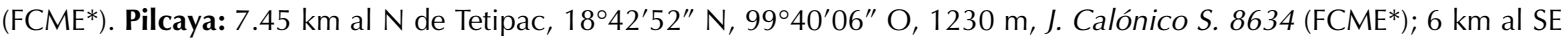
de Pilcaya, camino a Tenoxcotitlán, $18^{\circ} 42^{\prime} 30^{\prime \prime} \mathrm{N}, 99^{\circ} 40^{\prime} 20^{\prime \prime} \mathrm{O}, 1310 \mathrm{~m}$, R. Cruz-Durán et al. 1289 (FCME*); 9.87 km al NO de Tetipac, 1843'56" N, 9940'56" O, 1310 m, R. Cruz-Durán 2234 (FCME*). Taxco de Alarcón: 13 km sobre la

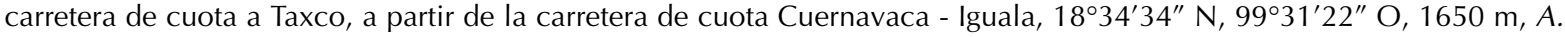
Espejo, A. R. López-Ferrari, J. Ceja, A. Mendoza e I. Ramírez 6151 (UAMIZ); hills near Taxco, 18³3’ N, $99^{\circ} 35$ W, D. C. Goebel s. n. (US). Teloloapan: 6 km al SE de Teloloapan, carretera Ciudad Altamirano - Iguala, $18^{\circ} 23^{\prime}$ N, $99^{\circ} 52^{\prime} \mathrm{O}^{\prime} 1500$

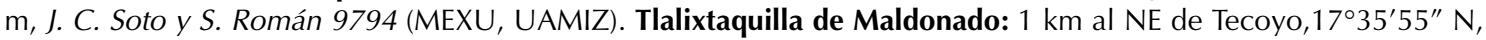
98²8'28" O, 1100 m, J. Calónico S. 1736 (FCME). Xochihuehuetlán: Tecoanapa, lado NE del cerro Xilotzin, 1759’ N, $98^{\circ} 28^{\prime} 48^{\prime \prime}$ O, 1300 m, E.Moreno G. y M. A. Monroy de la R, 515 (FCME).

48. Tillandsia fuchsii W.Till, Bromelie 1990:30-32, f. a-g. 1990.

Figura 16

DISTRIBUCIÓN: Guatemala y México.

ALTITUD: 800-1100 m.

EJEMPLARES EXAMINADOS: Coyuca de Catalán: Trincheras, $18^{\circ} 5^{\prime} \mathrm{N}, 101^{\circ} 16^{\prime} \mathrm{O}, \mathrm{G}$. B. Hinton 10126 (GH, US); 8 km al

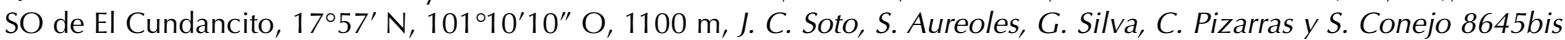
(MEXU). José Azueta: $20 \mathrm{~km}$ al NNE de Zihuatanejo, camino Zihuatanejo - Ciudad Altamirano, 17 $7^{\circ} 45^{\prime} 30^{\prime \prime} \mathrm{N}$, 10130'10" O, 280 m, F. Lorea 4034 (FCME); en San Antonio, a 13 km al NE de Vallecitos de Zaragoza, 175ㅗ' N, $101^{\circ} 15^{\prime}$ O, 980 m, J. C. Soto, S. Román y A. Vidal 12071 (MEXU). San Luis Acatlán: Mojonera de Atotonilco, 3 km al NO de Atotonilco o a $11 \mathrm{~km}$ al NO de Horcasitas, camino San Luis Acatlán - Pascala del Oro, $16^{\circ} 55^{\prime} \mathrm{N}, 9^{\circ} 51^{\prime} \mathrm{O}, 800$ m, E. Martínez, J. Victorio y B. Morales de Jesús 3538 (ENCB, GH, MEXU).

49. Tillandsia guerreroensis Rauh, Akad.Wiss.Abh.Math.-Naturwiss. KI.Trop.Subtrop.Pflanzenwelt. 60: 65-67 (967-969), f 45. 1987.

Figura 16

Distribución: Endémica de México.

ALTITUD: $100-500 \mathrm{~m}$.

EJEMPLARES EXAMINADOS: Tecpan de Galeana: Acapulco - Zihuatanejo “Zhijuantenajo", 17¹4' N, 10048’49" O, 100 m, P. Koide s. n. (HEID).

OTROS REGISTROS: Juan R. Escudero: Tierra Colorada, 179'56" N, 99³1'35" O, 500 m, L. Hromadnik 15080 (WU) (Till, com. pers.).

50. Tillandsia gymnobotrya Baker, J.Bot. 25: 243-244. 1887.

Figura 17

DISTRIBUCIÓN: Endémica de México.

ALTITUD: $2030 \mathrm{~m}$.

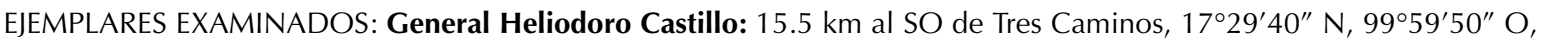
2030 m, J. Calónico S. 8433 (FCME*).

51. Tillandsia hintoniana L.B.Sm., Phytologia 28: 34-35, t. 1, f. I, J. 1974.

Figura 16 DISTRIBUCIÓN: Endémica de México.

ALTITUD: $1140-1785 \mathrm{~m}$.

EJEMPLARES EXAMINADOS: Chilpancingo de los Bravo: $20 \mathrm{~km}$ from Chilpancingo, $17^{\circ} 23^{\prime} \mathrm{N}, 99^{\circ} 20^{\prime} \mathrm{W}, \mathrm{ca} .4000 \mathrm{ft}, P$.

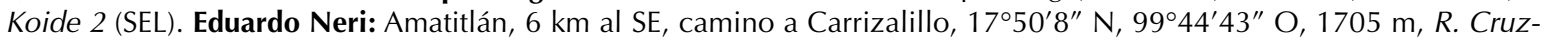

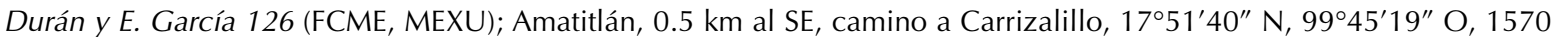

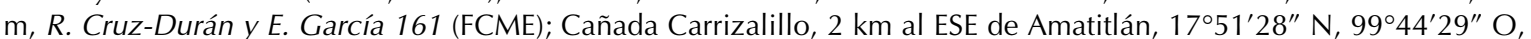
1560 m, R. Cruz-Durán y E. García 767 (FCME); al NE de Xochipala y El Mirabal, $17^{\circ} 44$ N, 9944' O, 1785 m, O. A.

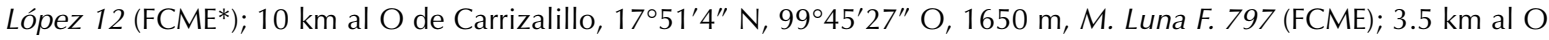
de Carrizalillo, $17^{\circ} 50^{\prime} 8^{\prime \prime} \mathrm{N}, 99^{\circ} 43^{\prime} 45^{\prime \prime} \mathrm{O}, 1720 \mathrm{~m}, \mathrm{M}$. A. Monroy de la R. 257 (FCME). Leonardo Bravo: $0.5 \mathrm{~km}$ al O de La Escalera, 1743'41" N, 9951'04" O, 1600 m, J. Calónico 8703 (FCME*). Zirándaro: 17 km al SE de Guayameo, $18^{\circ} 15^{\prime} \mathrm{N}, 101^{\circ} 6^{\prime} \mathrm{O}, 1140 \mathrm{~m}$, J. C. Soto, S. Román y F. Solórzano 12442 (MEXU).

52. Tillandsia ilseana W.Till, Halbritter y Zecher, J.Bromeliad Soc. 39: 153-156, f.3-6. 1989.

Figura 17 DISTRIBUCIÓN: Endémica de México.

ALTITUD: 1300-1600 m.

EJEMPLARES EXAMINADOS: Tixtla de Guerrero: along the road from Chilpancingo to Chilapa, $17^{\circ} 36^{\prime} \mathrm{N}, 9^{\circ} 21^{\prime} \mathrm{W}$, 1330-1600 m, E. e I. Zecher 8/80 (UAMIZ, WU).

53. Tillandsia intermedia Mez, Bull.Herb.Boissier sér. 2. 3: 141. 1903.

Figura 17 
DISTRIBUCIÓN: Endémica de México.

ALTITUD: 0-50 m.

EJEMPLARES EXAMINADOS: Acapulco de Juárez: Isla la Roqueta, Acapulco, $16^{\circ} 49^{\prime} 13^{\prime \prime} \mathrm{N}, 99^{\circ} 54^{\prime} 30^{\prime \prime} \mathrm{O}$, J. Mancilla C. 107 (FCME); J. C. Soto y S. Aureoles 8484 (MEXU, IEB); La Venta, 1654'30" N, 9948' O, 50 m, F. A. Villanueva s. $n$. (IEB). José Azueta: Zihuatanejo, orilla del mar, $17^{\circ} 38^{\prime} \mathrm{N}, 101^{\circ} 33^{\prime} \mathrm{O}, 0 \mathrm{~m}, \mathrm{~W}$. Boege 1629 (MEXU); E. Langlassé 370 $(\mathrm{GH}, \mathrm{P}, \mathrm{US})$; at the sea shore between playa La Ropa and playa Las Gatas, Zihuatanejo, $17^{\circ} 40^{\prime} \mathrm{N}, 101^{\circ} 38^{\prime} \mathrm{W}, 0 \mathrm{~m}, \mathrm{M}$. Yoshpa s. n. (SEL).

54. Tillandsia ionantha Planch., FI.Serres 10:101, t. 1006. 1855.

Figura 17 DISTRIBUCIÓN: México a Nicaragua.

ALTITUD: $250-1550 \mathrm{~m}$.

EJEMPLARES EXAMINADOS: Alpoyeca: 0.5 km al E de Tecoyo, 1736'25" N, 98³0'30" O, 1100 m, J. Calónico S. 1787 (FCME). Buenavista de Cuéllar: Cañón de la Mano, $18^{\circ} 24^{\prime} 56^{\prime \prime} \mathrm{N}, 9^{\circ} 29^{\prime} 50^{\prime \prime} \mathrm{O}$, A. Ramírez s. n. (MEXU). Chilpancingo

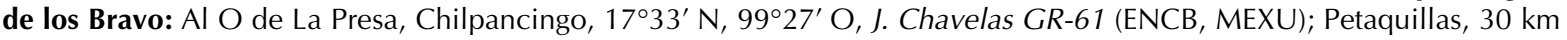
al S de Chilpancingo, carretera Chilpancingo - Acapulco, $17^{\circ} 29^{\prime} 30^{\prime \prime} \mathrm{N}, 99^{\circ} 27^{\prime} \mathrm{O}, 1220 \mathrm{~m}$, M. Flores, A. Martínez, S.

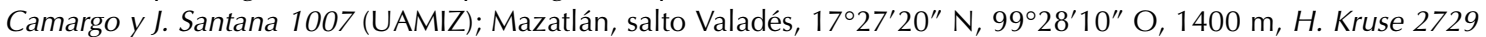

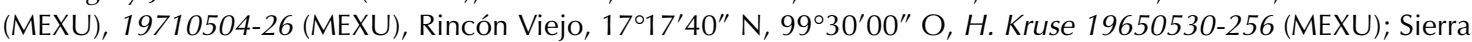

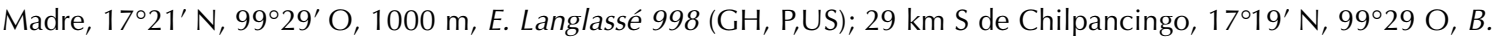
Leuenberger y C. Schiers 2726 (MEXU). Coyuca de Catalán: Santa Ana, 184' N, 101 ${ }^{\circ} 1^{\prime}$ O, 1260 m, G. B. Hinton et al. $10110(\mathrm{GH}, \mathrm{MEXU}, \mathrm{UC}), 10112(\mathrm{GH}, \mathrm{MEXU}, \mathrm{SEL}, \mathrm{US})$; El Cundancito, $15 \mathrm{~km}$ al NE de El Bálsamo, carretera Zihuatanejo - Ciudad Altamirano, 18 $8^{\circ} 1^{\prime} \mathrm{N}, 101^{\circ} 8^{\prime} 15^{\prime \prime} \mathrm{O}, 1120 \mathrm{~m}$, T. P. Ramamoorthy, E. Martínez y J. C. Soto 4298 (MEXU); 8 km al

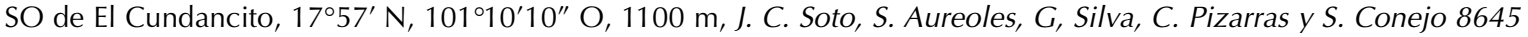
(MEXU). General Heliodoro Castillo-[Totolapan]: Campo Morado to Otatlán, $17^{\circ} 35^{\prime} \mathrm{N}, 100^{\circ} 11^{\prime} \mathrm{O}$, G. B. Hinton 14256

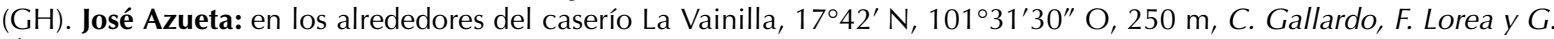
Ávila 485 (FCME, MEXU) $20 \mathrm{~km}$ al NNE de Zihuatanejo, camino Zihuatanejo - Ciudad Altamirano, 17 $45^{\circ} 30^{\prime \prime} \mathrm{N}$,

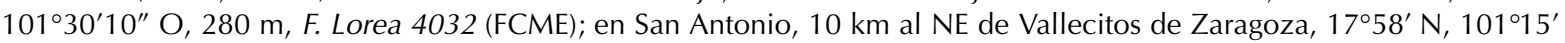

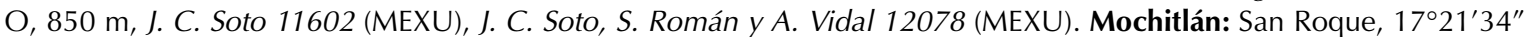

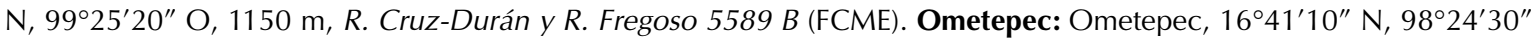
O, 390 m, G. Gaxiola 250 (FCME*). Pilcaya: 9.87 km al NO de Tetipac, 1843'56" N, 9940'546" O, $1310 \mathrm{~m}, R$. CruzDurán 2247 (FCME*); Corralejo, Cacahuamilpa, 1841' N, 99॰30’ O, O. Holguín s. n. (ENCB). Quechultenango: 3 km al $\mathrm{N}$ de Juxtlahuaca, ribera del río Blanco, $17^{\circ} 24^{\prime} 30^{\prime \prime} \mathrm{N}, 9^{\circ} 9^{\prime} 30^{\prime \prime} \mathrm{O}, \mathrm{D}$. Ascencio 144 (ENCB, IEB). Taxco de Alarcón: Atzala, $6.29 \mathrm{~km}$ al SO de Taxco de Alarcón, 18³0'52" N, 99³9'49" O, 1400 m, J. Calónico S. 8686 (FCME*); Atzala,

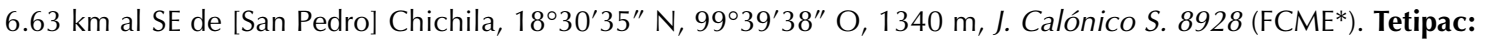
Chontalcuatlán, $18^{\circ} 39^{\prime} 5^{\prime \prime} \mathrm{N}, 99^{\circ} 34^{\prime} 0^{\prime \prime}$ O, F. Mata G. s. n. (FCME). Tixtla de Guerrero: ca. $5 \mathrm{~km}$ al SO de Tixtla, $17^{\circ} 33^{\prime} 10^{\prime \prime} \mathrm{N}, 9^{\circ} 26^{\prime} \mathrm{O}, 1550 \mathrm{~m}$, L. Cervantes 23 (FCME).

55. Tillandsia juncea (Ruiz et Pav.) Poir., in Lam., Encycl.Suppl. 5:309. 1817. DISTRIBUCIÓN: México Las Antillas, Centroamérica y norte de Sudamérica.

Figura 17 ALTITUD: 250-1700 m.

EJEMPLARES EXAMINADOS: Chilapa de Álvarez: ca. $5 \mathrm{~km}$ al SE de Atzacoaloya por el camino que va a Hueycaltenango, $17^{\circ} 31^{\prime} \mathrm{N}, 9^{\circ} 7^{\prime} 30^{\prime \prime} \mathrm{O}, 1545 \mathrm{~m}$, F. Lorea 1006 (FCME, UAMIZ). Chilpancingo de los Bravo: cerca de Chilpancingo, 17 $34^{\prime} \mathrm{N}, 9^{\circ} 30^{\prime} \mathrm{O}, 1500 \mathrm{~m}$, E. Matuda 38430 (MEXU). José Azueta: ca. $800 \mathrm{~m}$ al SO del caserío La Vainilla, por la cañada del arroyo La Vainilla, $17^{\circ} 42^{\prime} \mathrm{N}, 101^{\circ} 31^{\prime} 30^{\prime \prime} \mathrm{O}, 250 \mathrm{~m}, \mathrm{C}$. Gallardo et al. 695 (FCME, UAMIZ);

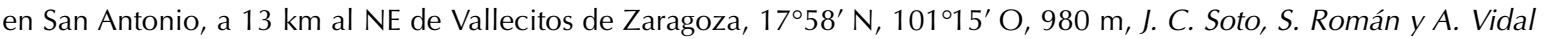

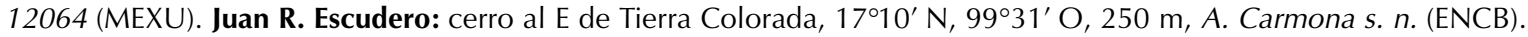
Malinaltepec: Malinaltepec, $17^{\circ} 15^{\prime} \mathrm{N}, 9^{\circ} 4^{\prime} \mathrm{O}, 1700 \mathrm{~m}$, I. Wagenbreth 540 (MEXU). Tixtla de Guerrero: La Estacada, $17^{\circ} 34^{\prime} 50^{\prime \prime} \mathrm{N}, 99^{\circ} 16^{\prime} 45^{\prime \prime} \mathrm{O}$, A. Hernández 164 (FCME).

OTROS REGISTROS: Coyuca de Catalán: Tierras Blancas, $18^{\circ} 9^{\prime} \mathrm{N}, 101^{\circ} 17^{\prime} \mathrm{O}$, G. B. Hinton 10142 (K) [Smith y Downs, 1977: 917].

56. Tillandsia kalmbacheri Matuda, Cact.Suc.Mex. 19:26, f. 16. 1974.

Figura 17

DISTRIBUCIÓN: Endémica de México.

ALTITUD: 900-1500 m.

EJEMPLARES EXAMINADOS: Chilpancingo de los Bravo: Cerro de los Encinos, cerca de Chilpancingo, $17^{\circ} 34^{\prime} \mathrm{N}, 9^{\circ} 30^{\prime}$

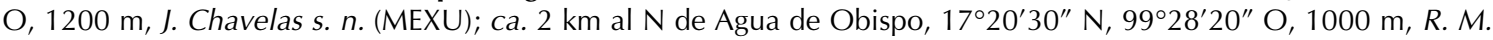
Fonseca 1708 (FCME*); filo de cerro Las Trincheras, entre Rincón de la Vía y Agua de Obispo, 17¹8'15" N, 99²8' O, 900 m, H. Kruse 3119 (MEXU, MO); H. Kruse [3119] sub Matuda 38629 (CODAGEM, LL, MEXU). Malinaltepec: 
Malinaltepec, $17^{\circ} 15^{\prime} \mathrm{N}, 98^{\circ} 40^{\prime} \mathrm{O}, 1500 \mathrm{~m}$, I. Wagenbreth 462 (MEXU), 463 (MEXU).

57. Tillandsia langlasseana Mez, Bull.Herb.Boissier sér. 2. 3: 142. 1903.

Figura 18

DISTRIBUCIÓN: Endémica de México.

ALTITUD: $700-2400 \mathrm{~m}$.

EJEMPLARES EXAMINADOS: Chilpancingo de los Bravo: Tezcaltitemi, Mazatlán, $17^{\circ} 26^{\prime} 30^{\prime \prime} \mathrm{N}, 99^{\circ} 27^{\prime} 30 \mathrm{O}^{\prime} \mathrm{H}^{\prime}$ Kruse

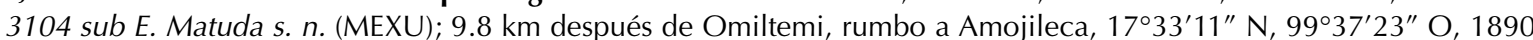
m, A. R. López-Ferrari, A. Espejo y J. Ceja 2836 (CICY, UAMIZ); a 2 km al O de Omiltemi, 17³3' N, 99²4' O, 2400 m,

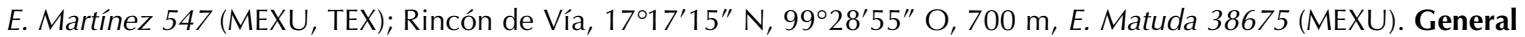
Heliodoro Castillo: Las Palomas, a 5 km al NO de Corralitos, $17^{\circ} 41^{\prime} 53^{\prime \prime}$ N, 9953'13" O, 2310 m, J. Calónico S. 7389 (FCME*). Leonardo Bravo: 2 km al S por la desviación a Jaleaca,17³2'29" N, 9953'57" O, 1890 m, J. Calónico S. 5169

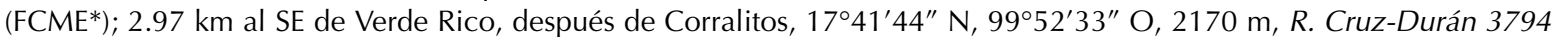
(FCME).

58. Tillandsia laui Matuda, Cact.Suc.Mex. 20:96-97, f. 48. 1975.

Figura 18 DISTRIBUCIÓN: Endémica de México.

ALTITUD: ca. $1500 \mathrm{~m}$.

EJEMPLARES EXAMINADOS: Ninguno.

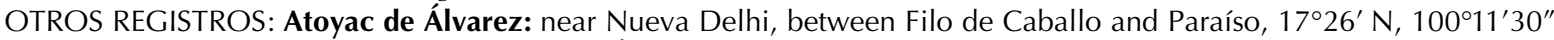
W, ca. 1500 m. [Ehlers, 1992: 74]. Chilapa de Álvarez: near Chilapa, 17³6’ N, 99¹0’ O, ca. 1500 m. [Ehlers, 1992: 74].

59. Tillandsia $\times$ Iydiae Ehlers, Bromelie 2000(2):57 (55-59; figs. 1-4; photogrs. 1-2). 2000.

Figura 18

$=$ Tillandsia caput-medusae $\times$ T. circinnatioides

DISTRIBUCIÓN: Endémica de México.

ALTITUD: 1000-1500 m.

EJEMPLARES EXAMINADOS: Chilapa de Álvarez: on roadside trees NE of Chilapa, $17^{\circ} 37^{\prime} \mathrm{N}, 9^{\circ} 10^{\prime} \mathrm{W}, \mathrm{ca} .1500 \mathrm{~m}, \mathrm{~A}$. Lau sub H. Luther s. n. (SEL). Eduardo Neri: along the road from Milpillas to Filo de Caballo, $1063 \mathrm{~m}, R$. Ehlers EM991901 (WU); between Milpillas and Filo de Caballo, 1748'40" N, 9940’ W, 1000 m, L. Köhres y G. Köhres s. n.

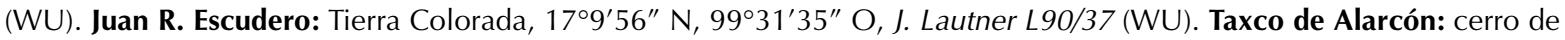

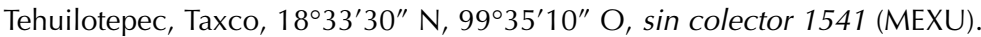

60. Tillandsia macdougallii L.B.Sm., Contr.U.S.Natl.Herb. 29: 277-278, f. 2. 1949.

Figura 18 DISTRIBUCIÓN: Endémica de México.

ALTITUD: 2270-2700 m.

EJEMPLARES EXAMINADOS: Chilpancingo de los Bravo: sobre el camino que parte de Omiltemi hacia El Cedral, 17³2'17" N, 9940’27" O, 2270 m, J. Ceja, A. Espejo, A. R. López-Ferrari, A. Mendoza, B. Pérez e I. Reyes 1004

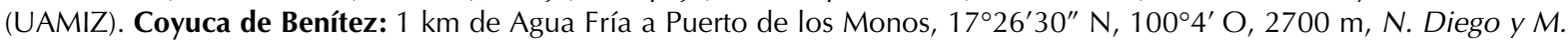
Castro 9273 (FCME, UAMIZ). Leonardo Bravo: 5.06 km al S de Tepozonalco, 17³3'31" N, 9944'06" O, $2400 \mathrm{~m}, J$. Calónico S. 9220 (FCME*).

61. Tillandsia magnusiana Wittm., Bot.Jahrb.Syst. 11:66. 1901; Phytologia 19: 287. 1970.

Figura 18 DISTRIBUCIÓN: México a Honduras y El Salvador.

ALTITUD: $2045 \mathrm{~m}$.

EJEMPLARES EXAMINADOS: Tlacoapa: El Tejocote (camino a Tlacoapa) a $54 \mathrm{~km}$ de Tlatlauquitepec, $2045 \mathrm{~m}$, J. Almazán 94 (FCME*), 95 (FCME*), 96 (FCME*).

62. Tillandsia makoyana Baker, Hand.Bromel. 188. 1889.

Figura 18

DISTRIBUCIÓN: Endémica de México.

ALTITUD: 0-1830 m.

EJEMPLARES EXAMINADOS: Atlixtac: Santa Isabel, cerca de Petatlán, 17³5'20" N, 990'16" O, 1200 m, R. Cruz-Durán et al. 5500 (FCME*); $1.86 \mathrm{~km}$ al S de Tozoyapan, 17³4'48" N, 9901'6 " O, $1550 \mathrm{~m}$, C. A. Hernández A. 34 (FCME*);

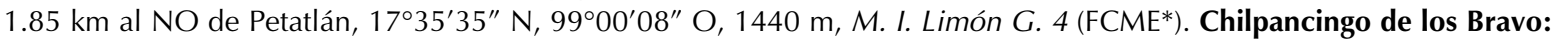

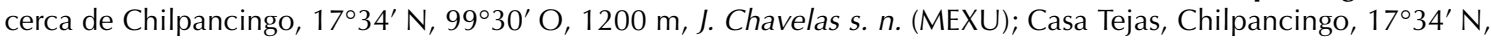
99³0’ O, 500 m, H. Kruse 3122 (MEXU); 14.8 km después de Omiltemi, rumbo a Amojileca, ca. $5 \mathrm{~km}$ antes de Amojileca, 17³4'34" N, 99³5'50" O, 1830 m, A. R. López-Ferrari, A. Espejo y J. Ceja 2840 (UAMIZ). José Azueta:

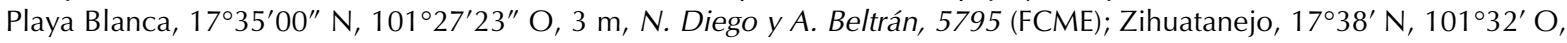


FLORA BROMELIOLÓGICA DE GUERRERO

E. Langlassé 274 (US). La Unión de Isidoro Montes de Oca: 2 km al N de Las Guacamayas, camino La Unión Coahuayutla, $18^{\circ} 1^{\prime} \mathrm{N}, 101^{\circ} 45^{\prime} \mathrm{O}, 120 \mathrm{~m}$, J. C. Soto, A. Román de Soto y F. Soto 9621 (MEXU). Leonardo Bravo: $0.5 \mathrm{~km}$

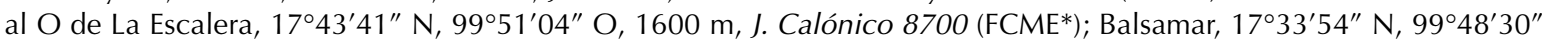

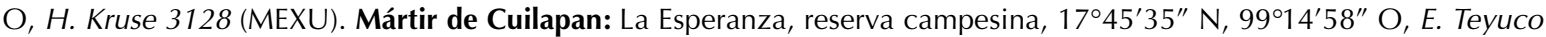

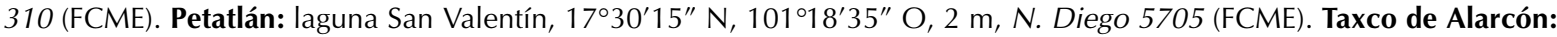
cerca de San Francisco Acuitlapan, camino a Las Granadas, 18³6' N, 99 32' O, $1400 \mathrm{~m}$, C. Beutelspacher s. n. sub E. Matuda 38372 (CODAGEM); $13 \mathrm{~km}$ sobre el camino a Taxco a partir de la carretera de cuota Cuernavaca - Iguala, 18³4'34" N, 99³1'22" O, 1650 m, A. Espejo, A. R. López-Ferrari, J. Ceja, A. Mendoza e I. Ramírez 6149 (IEB, UAMIZ). Tetipac: alrededores de Tetipac, 18³9'5" N, 99³8'30" O, 1620 m, A. Espejo, A. R. López-Ferrari, J. Ceja, A. Mendoza e I. Ramírez 6164 (UAMIZ). Tixtla de Guerrero: ladera N del cerro Xomislo, ca. $10 \mathrm{~km}$ por el camino que va de Tixtla a

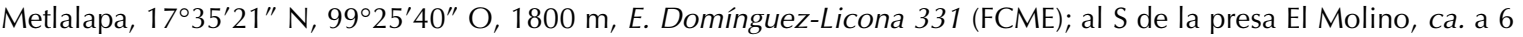
$\mathrm{km}$ al $\mathrm{O}$ de Tixtla, alrededores de la presa y cerro de la Media Luna, $17^{\circ} 33^{\prime} 23^{\prime \prime} \mathrm{N}, 9^{\circ} 26^{\prime} 27^{\prime \prime} \mathrm{O}, 1700 \mathrm{~m}$, R. M. Fonseca

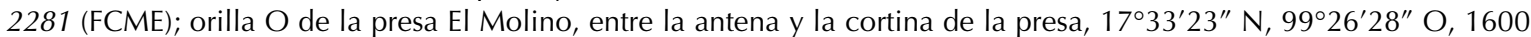
m, R. M. Fonseca 3130 (FCME, UAMIZ). Xochihuehuetlán: paraje El Agua Zarca "La Huazarca", 2.5 km al NO de

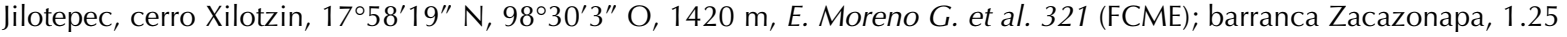
$\mathrm{km}$ al NNE de Jilotepec, $17^{\circ} 58^{\prime} 34^{\prime \prime} \mathrm{N}, 9^{\circ} 28^{\prime} 45^{\prime \prime} \mathrm{O}, 1260 \mathrm{~m}$, E. Moreno G. et al. 861 (FCME). Zirándaro: $14 \mathrm{~km}$ al SO de El Tálamo, carretera Zirándaro - Guayameo, 710 m, J. C. Soto N. y G. Silva R. 4368 (MEXU). Zitlala: Topiltepec, reserva campesina, 1739' $1^{\prime \prime} \mathrm{N}, 9^{\circ} 13^{\prime} 2^{\prime \prime} \mathrm{O}, 1500 \mathrm{~m}$, C. Miranda 320 (FCME), N. Diego 7790 (FCME).

63. Tillandsia marabascoensis Ehlers et Lautner, Bromelie 1992:69-71, figs. 1992. DISTRIBUCIÓN: Endémica de México.

ALTITUD: $100-240 \mathrm{~m}$

EJEMPLARES EXAMINADOS: José Azueta: al SO del caserío La Vainilla, por la cañada, $17^{\circ} 42^{\prime} \mathrm{N}, 101^{\circ} 31^{\prime} 30^{\prime \prime} \mathrm{O}, 240 \mathrm{~m}$, C. Gallardo. F. Lorea y A. Hanan 269 (FCME, MEXU). La Unión de Isidoro Montes de Oca: [hacienda] El Tibor, 1755' $\mathrm{N}, 101^{\circ} 40^{\prime} \mathrm{O}$, E. Langlassé 297 (GH, US). Petatlán: $5 \mathrm{~km}$ al N de Coyuquilla [sic], $17^{\circ} 28^{\prime} \mathrm{N}, 101^{\circ} 2^{\prime} \mathrm{O}, 100 \mathrm{~m}, J . \mathrm{C}$ Soto, S. Román y A. Vidal 12110 (MEXU, UAMIZ).

64. Tillandsia maritima Matuda, Cact.Suc.Mex. 16:90-91, f. 47. 1971.

Figura 16 DISTRIBUCIÓN: Endémica de México.

ALTITUD: 0-220 (-1500) m.

EJEMPLARES EXAMINADOS: Acapulco de Juárez: in area of Pie de la Cuesta, near Acapulco, 16 $54^{\prime} \mathrm{N}^{\prime} 9^{\circ} 57^{\prime} 45^{\prime \prime} \mathrm{W}, F$ A. Barkley 14199 (MEXU); vivero El Huayacán, La Poza, 1652' N, 9954’ O, W. López-Forment 904 (MEXU), 1281 (MEXU); terreno frente a gasolinería Revolcadero, $16^{\circ} 52^{\prime} \mathrm{N}, 9^{\circ} 54^{\prime} \mathrm{O}, 0 \mathrm{~m}, \mathrm{~W}$. López-Forment 1325 (ENCB, MEXU); en

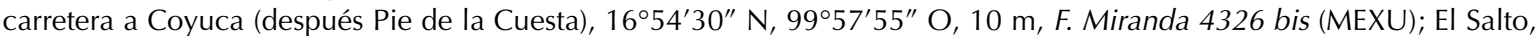

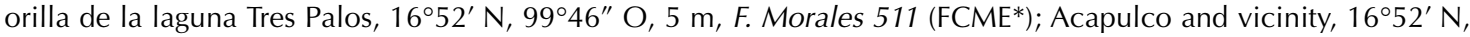
9954' O, E. Palmer 547 (US). Azoyú: en arroyo Totola, 1 km al N de El Aguacate, camino Marquelia - Agua Zarca - San Luis Acatlán, $16^{\circ} 40^{\prime} \mathrm{N}, 98^{\circ} 42^{\prime} \mathrm{O}, 220 \mathrm{~m}$, E. Martínez y B. Morales de Jesús 3386 (MEXU). Chilpancingo de los Bravo: Mazatlán, Salto Valadés, $17^{\circ} 27^{\prime} 20^{\prime \prime} \mathrm{N}, 9^{\circ} 28^{\prime} 10^{\prime \prime} \mathrm{O}, 1500 \mathrm{~m}, \mathrm{H}$. Kruse 3087 (FCME, MEXU). José Azueta: Salinas del

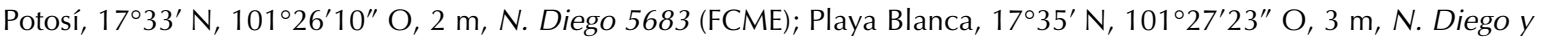

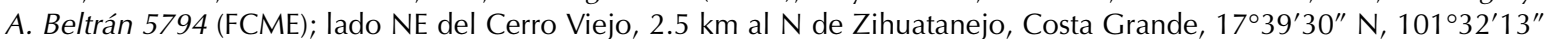
$\mathrm{O}, 105 \mathrm{~m}$, S. Peralta 471 (FCME). La Unión de Isidoro Montes de Oca: ca. $14 \mathrm{~km}$ al $\mathrm{N}$ de Coyuquilla, rumbo a Infiernillo, por la carretera de cuota Lázaro Cárdenas - Uruapan, $18^{\circ} 2^{\prime} 21^{\prime \prime} \mathrm{N}, 101^{\circ} 56^{\prime} 46^{\prime \prime} \mathrm{O}, 116 \mathrm{~m}, A$, Espejo, A. R. López-Ferrari, J. Ceja, A. Mendoza R. e I. Ramírez M. 6597 (UAMIZ). Petatlán: La Salada, 17³2'26" N, 101²6'15" O,

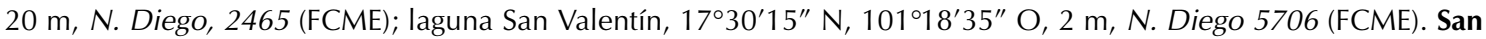
Marcos: along route 200, ca. 29 miles east of Acapulco, R. Merrill King 4191 (MICH, US). Sin municipio indicado: entre Acapulco (Gro.) y Pinotepa (Oax.), E. Matuda 38288 (MEXU, US).

65. Tillandsia mirabilis L.Hrom., Bromelie 1992:27-29, figs. 1992.

Figura 19 DISTRIBUCIÓN: Endémica de México.

ALTITUD: ca. $1500 \mathrm{~m}$.

EJEMPLARES EXAMINADOS: Atlixtac: in rupibus verticalibus prope fluminem "río Petatlán" ad occidentalem solem pagi

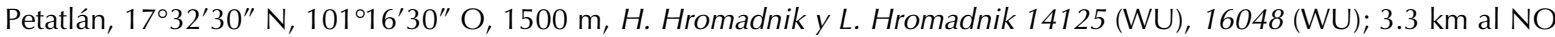
de Petatlán, 17³5'39" N, 9900'53" O, 1500 m, L. Mendizábal B. 34 (FCME*).

66. Tillandsia oaxacana L.B.Sm., Contr.U.S.Natl.Herb. 29: 279-280, f. 4. 1949.

Figura 19 DISTRIBUCIÓN: Endémica de México.

ALTITUD: 2520-3100 m. 
EJEMPLARES EXAMINADOS: Zapotitlán Tablas: a 1 km de Iztlahuazaca, rumbo a Tlacoapa, $17^{\circ} 20^{\prime} \mathrm{N}, 98^{\circ} 47^{\prime} \mathrm{O}, 2520$ m, E. Velázquez M. 124 (FCME*).

67. Tillandsia ortgiesiana E.Morren ex Mez, in C.DC., Monogr.Phan. 9: 678. 1896.

Figura 19 DISTRIBUCIÓN: Endémica de México.

ALTITUD: 0-20 m.

EJEMPLARES EXAMINADOS: Acapulco de Juárez: $2.5 \mathrm{~km}$ al O de Puerto Marqués, $16^{\circ} 49^{\prime} \mathrm{N}, 99^{\circ} 50^{\prime} 45^{\prime \prime} \mathrm{O}, 20 \mathrm{~m}, W$. López-Forment 494 (MEXU, TEX), 537 (MEXU, TEX); Acapulco, J. Marnier-Lapostolle s. n. (US), W. Rauh RM-15855 (US).

68. Tillandsia paraisoensis Ehlers, J.Bromeliad Soc. 42: 55-59, f. 3-5. 1992.

Figura 19 DISTRIBUCIÓN: Endémica de México.

ALTITUD: $760-1800 \mathrm{~m}$.

EJEMPLARES EXAMINADOS: Atoyac de Álvarez: along the street from (Nueva Dehli) Puerto del Gallo - El Paraíso, near El Paraíso, 17 $24^{\prime} 34^{\prime \prime} \mathrm{N}, 100^{\circ} 11^{\prime} 30^{\prime \prime}$ W, 1500 m, R. Ehlers y K. Ehlers EM 901003 (WU). Chilpancingo de los Bravo: en

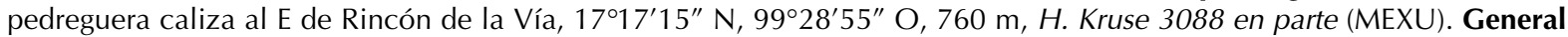
Heliodoro Castillo: Puente de la Dama, $17^{\circ} 30^{\prime} 11^{\prime \prime} \mathrm{N}, 9^{\circ} 59^{\prime} 04^{\prime \prime} \mathrm{O}, 1700 \mathrm{~m}, \mathrm{~N}$. Diego, A. Acosta y G. Fernández 8521

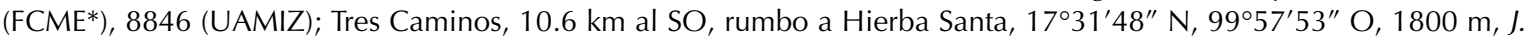
Calónico S. 8844 (FCME*), 8845 (FCME*).

69. Tillandsia pentasticha Rauh et Wülfinghoff, in Rauh, Akad.Wiss.Abh.Math.-Naturwiss. KI.Trop. Subtrop.Pflanzenwelt. 65:59-61 (685-687), f. 37-38. 1988.

Figura 19

DISTRIBUCIÓN: Endémica de México.

ALTITUD: $1495-2700 \mathrm{~m}$.

EJEMPLARES EXAMINADOS: Chilapa de Álvarez: $6 \mathrm{~km}$ antes de Hueycaltenango, por la carretera Atzacoaloya-

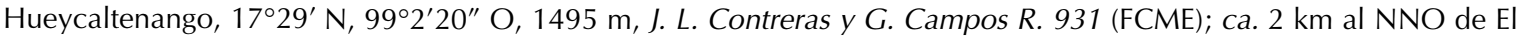
Caracol, camino Atzacoaloya - Hueycaltenango, $17^{\circ} 29^{\prime} \mathrm{N}, 9^{\circ} 4^{\prime} 30^{\prime \prime} \mathrm{O}, 1930 \mathrm{~m}$, F. Lorea 1223 (FCME, UAMIZ);

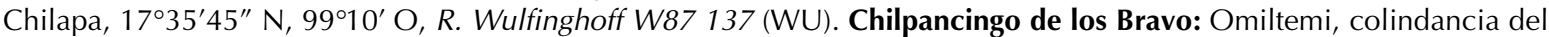
cerro El Conejo y El Retaje, 17³2'50" N, 9942' O, 2260 m, C. González 339 (FCME); 3.5 km después de Omiltemi,

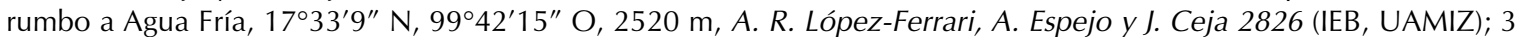
miles SE of Omiltemi, 17 $32^{\prime} 45^{\prime \prime} \mathrm{N}, 99^{\circ} 41^{\prime} 55^{\prime \prime} \mathrm{W}, 7800 \mathrm{ft}, \mathrm{T}$. W. McCorde y C. M. Rowell 3449 (MICH); $4 \mathrm{millas} \mathrm{O}$ de

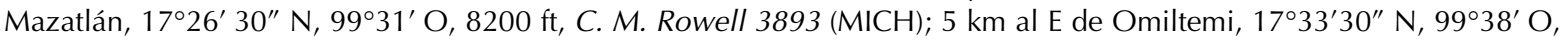
2300 m, J. Rzedowski 15979 (ENCB, US). Coyuca de Benítez: 3.85 km al S de Agua Fría, Cerro Tlacotepec, 17²4'39"

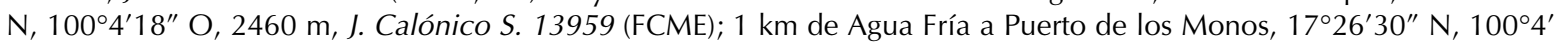
O, 2700 m, N. Diego y M. Castro 9265 (FCME, UAMIZ). Leonardo Bravo: 2 km adelante de Cruz de Ocote, 17³3' N,

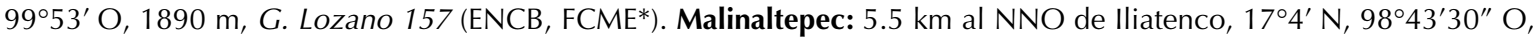

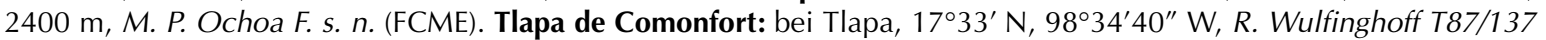
(HEID).

70. Tillandsia polystachia (L.) L., Sp.Pl. ed. 2. 410. 1762.

Figura 22

DISTRIBUCIÓN: Estados Unidos, México, Antillas, Centro y Sudamérica.

ALTITUD: ca. $700 \mathrm{~m}$.

EJEMPLARES EXAMINADOS: Chilpancingo de los Bravo: Rincón Viejo, arroyo frente a la bomba, $17^{\circ} 17^{\prime} \mathrm{N}^{\prime} 9^{\circ} 28^{\prime} \mathrm{O}^{\circ}$ 700 m, H. Kruse 825 (FCME, MEXU).

71. Tillandsia prodigiosa (Lem.) Baker, J.Bot. 26:140. 1888.

Figura 20

DISTRIBUCIÓN: Endémica de México.

ALTITUD: 800-2490 m.

EJEMPLARES EXAMINADOS: Atoyac de Álvarez: vic. of Nueva Dehli, $17^{\circ} 26^{\prime} \mathrm{N}, 100^{\circ} 11^{\prime} 30^{\prime \prime} \mathrm{W}, 1700 \mathrm{~m}, \mathrm{~K}$. Ehlers y R.

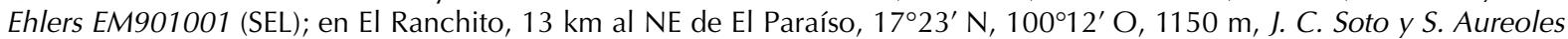
7867 (MEXU). Chilpancingo de los Bravo: Rincón de la Vía, $17^{\circ} 17^{\prime} 15^{\prime \prime} \mathrm{N}, 9^{\circ} 28^{\prime} 55^{\prime \prime} \mathrm{O}, 800$ m, H. Kruse s. n. sub E.

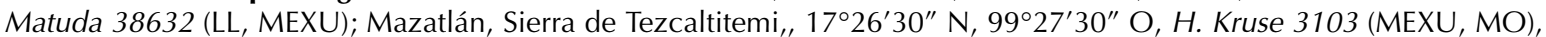

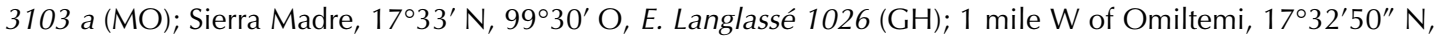

9941'30" O, 7800 ft, T. W. McCorde y C. M. Rowell, Jr. 3451 (MICH). General Heliodoro Castillo: Tres Caminos, 13.5

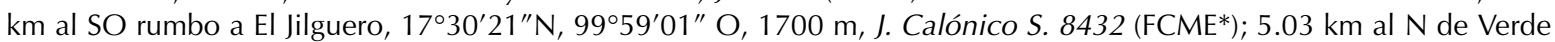

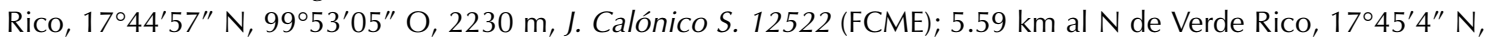
9952'49" O, 2200 m, J. Calónico S. 12601 (FCME); 8 km al SO del puerto El Jilguero, camino a Puerto del Gallo, 
FLORA BROMELIOLÓGICA DE GUERRERO

$17^{\circ} 28^{\prime} \mathrm{N}, 100^{\circ} 02^{\prime} 34^{\prime \prime} \mathrm{O}$, F. Lorea 3938 (FCME); Yerba Buena, sierra costal de Guerrero, 17³1' N, $99^{\circ} 58^{\prime} \mathrm{O}, 1900 \mathrm{~m}, E$. Matuda 38654 (MEXU). Ixcateopan de Cuauhtémoc: San Miguel, 2.5 km al N, camino Ixcateopan - Taxco, 18³0’36” N,

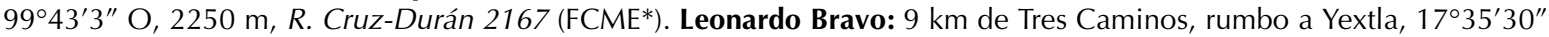
N, 9956'20" O, 2050 m, J. Calónico S. 3549 (FCME*); ca. 2.5 km adelante de Carrizal de Bravos, rumbo a Atoyac, $17^{\circ} 36^{\prime} \mathrm{N}, 9^{\circ} 50^{\prime} \mathrm{O}, 2400 \mathrm{~m}$, A. R. López-Ferrari, A. Espejo y T. Chehaibar 362 (UAMIZ); 5 km al SE de El Carrizal de los Bravos, camino Filo de Caballo - Chichihualco, 17³5' N, 9947' O, 2490 m, E. Martínez, T. P. Ramamoorthy y J. Miller

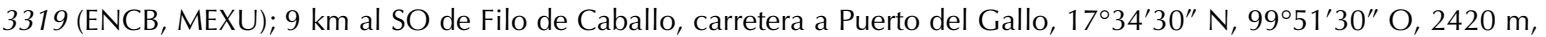

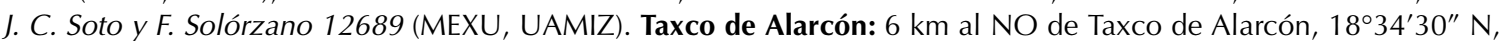

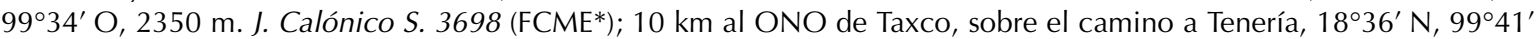
$\mathrm{O}, 2300 \mathrm{~m}$, J. Rzedowski 25242 a (ENCB). Sin municipio indicado: along the road from Milpillas to Nueva Dehli, $H . y L$. Hromadnik 14068 (UAMIZ); Vientos Fríos - Yerba Buena, 1800 m, R. Ehlers y K. Ehlers EM900906 (WU).

72. Tillandsia pseudobaileyi C.S.Gardner, Selbyana 7:363, f. 2, 4, 5. 1984.

Figura 20 DISTRIBUCIÓN: Endémica de México.

ALTITUD: 116-1430 m.

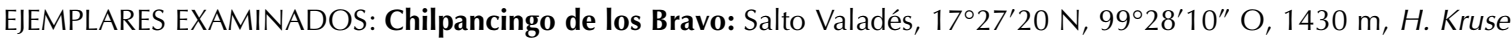
2704 (FCME, MEXU). La Unión de Isidoro Montes de Oca: ca. 14 km al N de Coyuquilla, rumbo a Infiernillo, por la carretera de cuota Lázaro Cárdenas - Uruapan, $18^{\circ} 2^{\prime} 21^{\prime \prime} \mathrm{N}, 101^{\circ} 56^{\prime} 46^{\prime \prime} \mathrm{O}, 116 \mathrm{~m}$, A. Espejo, A. R. López-Ferrari, J. Ceja, A. Mendoza e I. Ramírez 6598 (UAMIZ). Quechultenango: $2 \mathrm{~km}$ al N de Juxtlahuaca, rivera del río Blanco, $17^{\circ} 24^{\prime} 30^{\prime \prime}$ $\mathrm{N}, 9^{\circ} 9^{\prime} 30^{\prime \prime} \mathrm{O}$, D. Ascencio 145 (ENCB).

73. Tillandsia pseudosteacea Ehlers et Rauh, in Rauh, Trop.Subtrop.Pflanzenwelt. 58:35, f. 21. 1986. DISTRIBUCIÓN: Endémica de México.

ALTITUD: $710-2200 \mathrm{~m}$.

EJEMPLARES EXAMINADOS: Chilpancingo de los Bravo: Rincón Viejo, 17¹7'40" N, 99³0’ O, 710 m, H. Kruse 824

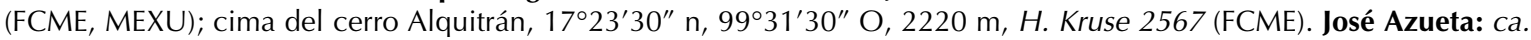

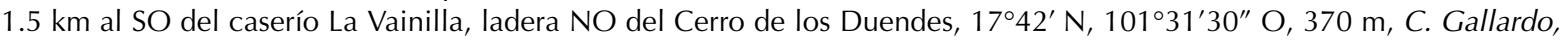
F. Lorea y A. Vargas 654 (FCME, IEB, MEXU, UAMIZ).

74. Tillandsia quaquaflorifera Matuda, Cact.Suc.Mex. 22:23-24, f. 14. 1977.

Figura 20 DISTRIBUCIÓN: Endémica de México.

ALTITUD: $1500-3280 \mathrm{~m}$.

EJEMPLARES EXAMINADOS: Atlixtac: cerca de Petatlán, 17³5' N, 9859'20" O, 1500 m, E. Matuda 38699

(CODAGEM, MEXU, MO, US), 38701 (ENCB, MEXU, MO). Atoyac de Álvarez: desviación El Iris, carretera Puerto del Gallo - Paraíso, $17^{\circ} 21^{\prime} \mathrm{N}, 100^{\circ} 12^{\prime} 55^{\prime \prime} \mathrm{O}, 2140 \mathrm{~m}$, J. Juárez G. s. n. (FCME*); El Parteaguas, 8 km después de la

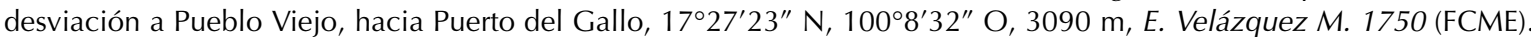
Coyuca de Benítez: Puerto Unión, ladera cerro Teotepec, $17^{\circ} 26^{\prime} 30^{\prime \prime} \mathrm{N}, 100^{\circ} 4^{\prime} 0^{\prime \prime} \mathrm{O}, 3030 \mathrm{~m}, \mathrm{~N}$. Diego, B. Ludlow y J.

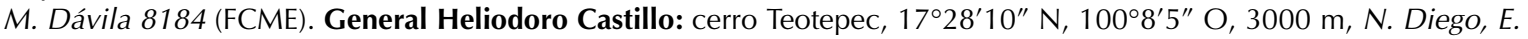
Acosta y G. Fernández 8118 (FCME); cima cerro Teotepec, antena de transmisiones (telefónica), ladera S, 17 $28^{\circ} 12^{\prime \prime} \mathrm{N}$, 1008'9" O, 3280 m, E. Domínguez-Licona 1219 (FCME); Teotepec a Puerto San Juan, 17²8' N, 1009' O, 3000 m, H.

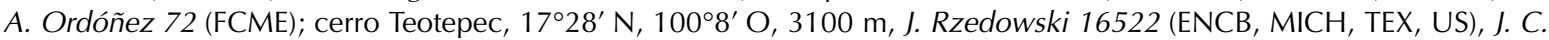
Soto y S. Aureoles 8423 (MEXU, UAMIZ); la antena de microondas, hacia el paraje Las Casitas, ladera poniente Teotepec, 17²8'35" N, 1009'8" O, 3100, E. Velázquez 1975 (FCME). Leonardo Bravo: Asoleadero, about $15 \mathrm{~km}$ W of Camotla, about $45 \mathrm{~km} \mathrm{~W}$ of Chichihualco, $17^{\circ} 37^{\prime} \mathrm{N}, 99^{\circ} 50^{\prime} \mathrm{O}$, C. Feddema 2852-A (MICH).

75. Tillandsia recurvata (L.) L., Sp.P. ed. 2. 410. 1762.

Figura 20

DISTRIBUCIÓN: Estados Unidos a Argentina.

ALTITUD: $700-1730 \mathrm{~m}$.

EJEMPLARES EXAMINADOS: Atlixtac: 0.48 km al SE de Petatlán, 17³4'38" N, 9859’30" O, 1480 m, M. Elías González 18 (FCME*); ca. 4 km después de Atlixtac, rumbo a Petatlán, 17³4’41" N, 9859’31" O, 1500 m, L. Mendizábal 59 (FCME*). Buenavista de Cuéllar: Cañón de la Mano, entre Los Amates y El Naranjo, $10 \mathrm{~km}$ al N de Iguala por el ferrocarril, al SO de Amates, sobre cerro, 18²4'56" N, 99²9'50" O, C. Catalán, F. Terán y S. Vázquez 548 (MEXU); estación de microondas del canal 13, $19 \mathrm{~km}$ al NE de Iguala, $18^{\circ} 24^{\prime} \mathrm{N}, 9^{\circ} 28^{\prime} \mathrm{O}$, A. Fernández y H. Ayala 6 (UAMIZ); Palmilla, 19 km al SE de Amacuzac, 18³2' N, 99²5' O, 1100 m, A. R. López-Ferrari 354 (CIIDIR, MEXU, UAMIZ); en Cieneguillas, $11 \mathrm{~km}$ al SO de Buenavista, $18^{\circ} 25^{\prime} \mathrm{N}, 9^{\circ} 29^{\prime} \mathrm{O}$, J. C. Soto y S. Aureoles 8755 (MEXU). Chilpancingo de los Bravo: ca. 5 km al N de Chilpancingo, carretera Chilpancingo - Iguala, $17^{\circ} 36^{\prime} \mathrm{N}, 9^{\circ} 31^{\prime} \mathrm{O}, 1375 \mathrm{~m}$, M. Flores, A. Martínez, S, 
Valeria Angélica Pulido-Esparza, Ana Rosa López-Ferrari y Adolfo Espejo-Serna

Camargo y J. Santana 1012 (UAMIZ); Chilpancingo, zona NE, 17³3’ N, 99³0’30” O, González 354 (MEXU); crescit locis alsis prope Chilpancingo Mexicanorum, $17^{\circ} 33^{\prime} \mathrm{N}, 99^{\circ} 30^{\prime} 30^{\prime \prime} \mathrm{O}$, A. Humboldt y A. Bonpland n. s. (P). Eduardo

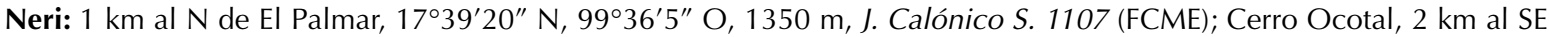
de Amatitlán, 1751'9" N, 9945’2" O, 1730 m, R. Cruz-Durán y M. E. García G. 779 (FCME); Xochipala, camino a La Laguna, 1747'30" N, 9942'20' O, 1400 m, C. Franco G. 17 (FCME); Cañón del Zopilote, desviación a Filo de Caballo, carretera México - Acapulco, 1748’ N, 99³3'40” O, 700 m, L. García-Martínez 14 (FCME); Tlacuitlapa (rancho abandonado hacia 1990), vertiente expuesta al NE del cerro Cacalotepec, a $1.5 \mathrm{~km}$ al N del pueblo de Ameyaltepec, a $80 \mathrm{~m}$ al N del manantial, 1759' N, 99³0' O, 880 m, G. Hall y J. Amith 335 (FCME); La Curva, carretera Zumpango Chilpancingo, en el km 259 Zumpango del Río, 17³7'40" N, 99³2'30" O, H. Kruse 2525 (FCME), 3113 (FCME,

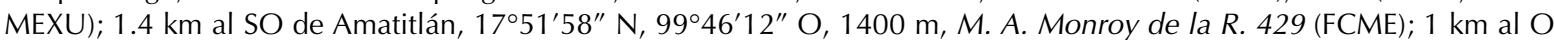

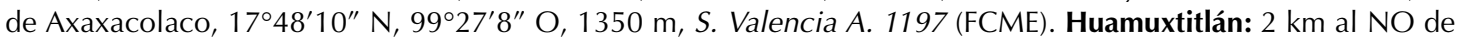

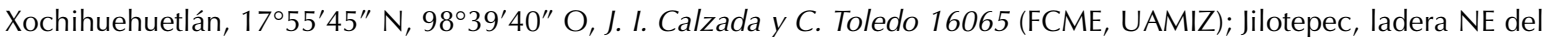

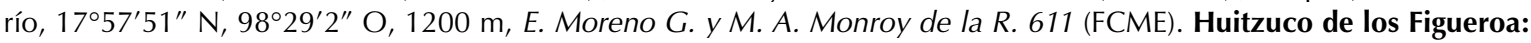

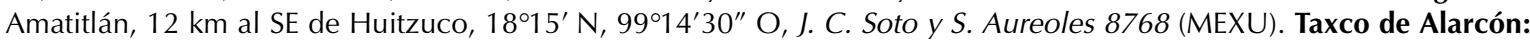
$1.5 \mathrm{~km}$ al SE de Tehuilotepec, 18³2'16" N, 99³2'14" O, 1550 m, J. Calónico S. 47610 bis (FCME*); 14 km sobre la

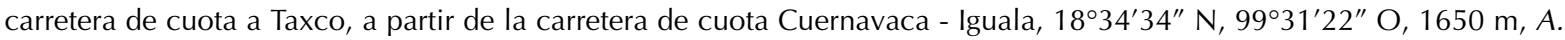
Espejo, A. R. López-Ferrari, J. Ceja, A. Mendoza e I. Ramírez 6152 (UAMIZ). Tixtla de Guerrero: Tixtla "Tiestla",

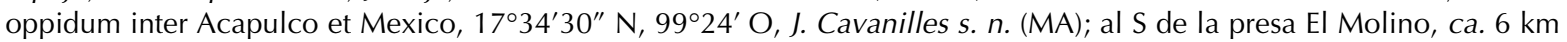

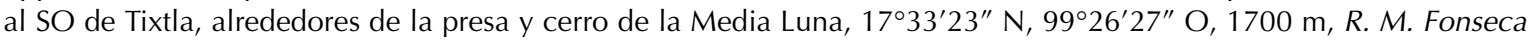
2279 (FCME, UAMIZ); orilla O de la presa El Molino, entre la antena y la cortina de la presa, $17^{\circ} 33^{\prime} 23^{\prime \prime} \mathrm{N}^{\circ}, 9^{\circ} 26^{\prime} 28^{\prime \prime}$

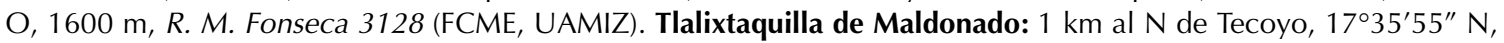
98²8'28" O, 1000 m, J. Calónico S. 1729 (FCME). Tlapa de Comonfort: 4 km al N de Tlapa, sobre la carretera Tlapa Alpoyeca, 17³4' N, 98³3' O, 1080 m, M. Flores, A. Martínez, J. Santana y S. Camargo 935 (UAMIZ). Xochihuehuetlán: $12 \mathrm{~km}$ al S de Jilotepec, carretera a Huamuxtitlán, $17^{\circ} 51^{\prime} 40^{\prime \prime} \mathrm{N}, 98^{\circ} 30^{\prime} 30^{\prime \prime} \mathrm{O}, 1090 \mathrm{~m}$, R. Fragoso y J. Santana 1438 (FCME*, UAMIZ).

OTROS REGISTROS: Coyuca de Catalán: Trincheras, 185' N, 101¹6’ O, G. B. Hinton 10128 (US) [Smith y Downs, 1977: 891].

76. Tillandsia rettigiana Mez, in C.DC., Monogr.Phan. 9: 704-705. 1896.

Figura 20

DISTRIBUCIÓN: Endémica de México.

ALTITUD: ca. $2 \mathrm{~m}$.

EJEMPLARES EXAMINADOS: Benito Juárez: Isla de los Tamarindos, laguna de Mitla, $17^{\circ} 0^{\prime} 5^{\prime \prime} \mathrm{N}, 100^{\circ} 14^{\prime} 16^{\prime \prime} \mathrm{O}, 2 \mathrm{~m}, L$. Lozada P. 1156 A (UAMIZ).

77. Tillandsia schiedeana Steud., Nomecl.Bot. ed. 2. 2:688. 1841.

Figura 21

DISTRIBUCIÓN: México, Las Antillas, Centroamérica y norte de Sudamérica

ALTITUD: 105-1900 m.

EJEMPLARES EXAMINADOS: Acapulco de Juárez: ca. 46 km después de Acapulco, rumbo a Chilpancingo. Río

Xaltianguis, $17^{\circ} 6^{\prime} 30^{\prime \prime} \mathrm{N}, 99^{\circ} 42^{\prime} 30^{\prime \prime} \mathrm{O}, 500 \mathrm{~m}$, A. R. López-Ferrari y A. Espejo 1749 bis (UAMIZ); vivero El Guayacán, La Poza, 1652' N, 9954' O, W. López-Forment 948 (MEXU, XAL). Ahuacuotzingo: Ajuatetla, reserva campesina, 1747’ N, 994'5" O, 1900 m, N. Diego 7774 (FCME, UAMIZ), N. Diego y M. Castro 9212 (FCME, UAMIZ). Alpoyeca: 0.5 km al

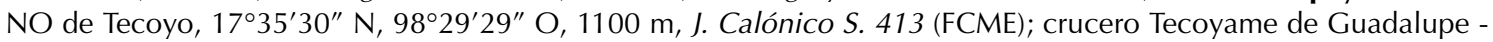
Tecoyo, 17³6' $5^{\prime \prime} \mathrm{N}, 98^{\circ} 29^{\prime} 29^{\prime \prime} \mathrm{O}, 1100 \mathrm{~m}$, J. Calónico S. 753 (FCME); 4 km al S de Ixcateopan, río Tlapaneco,

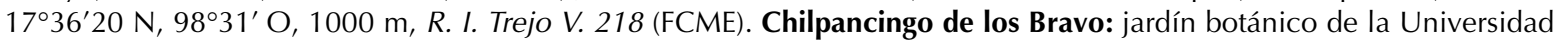

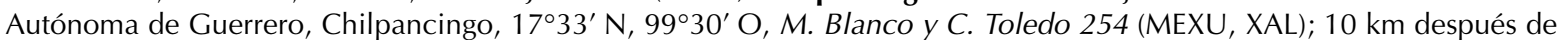
Chilpancingo, sobre la carretera a Tixtla, $17^{\circ} 29^{\prime} 58^{\prime \prime} \mathrm{N}, 9^{\circ} 26^{\prime} 30^{\prime \prime} \mathrm{O}, 1480 \mathrm{~m}$, J. L. Contreras J. y G. Campos R. 898

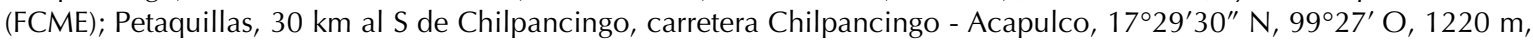

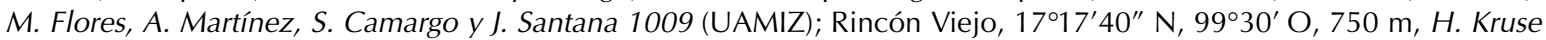

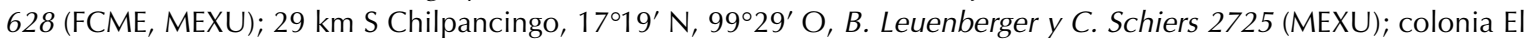
Ocotito - Jaleaca, km 3, 17²8' N, 9953' O, E. Martínez y O. Téllez 155 (MEXU, XAL); barranca cerca de Chilpancingo, $17^{\circ} 34^{\prime} \mathrm{N}, 99^{\circ} 30^{\prime} \mathrm{O}, 1200 \mathrm{~m}$, E. Matuda 38419 (MEXU, XAL); $28 \mathrm{~km}$ al S de Milpillas, sobre la carretera Iguala Chilpancingo, $17^{\circ} 32^{\prime} \mathrm{N}, 99^{\circ} 30^{\prime} \mathrm{O}, 900 \mathrm{~m}$, J. C. Soto y S. Aureoles 8897 (MEXU). Coahuyautla de José María Izazaga:

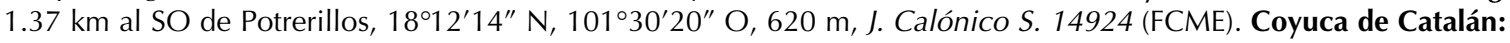
Trincheras, $18^{\circ} 5^{\prime} \mathrm{N}, 101^{\circ} 16^{\prime} \mathrm{O}$, G. B. Hinton et al. 10133 (MEXU); en El Cundancito, $94 \mathrm{~km}$ al SO de Ciudad Altamirano, $18^{\circ} 1^{\prime} \mathrm{N}, 101^{\circ} 8^{\prime} 15^{\prime \prime} \mathrm{O}, 1060 \mathrm{~m}$, E. Martínez y J. C. Soto 3673 (MEXU); $8 \mathrm{~km}$ al SO de El Cundancito, 1757' N, 101 $10^{\prime} 10^{\prime \prime}$ O, 1100 m, J. C. Soto, S. Aureoles, G. Silva, C. Pizarras y S. Conejo 8639 (MEXU). Eduardo Neri: 2 km al 
FLORA BROMELIOLÓGICA DE GUERRERO

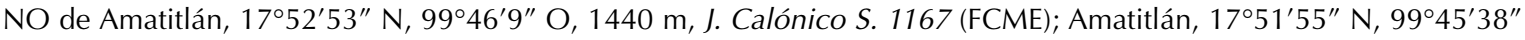

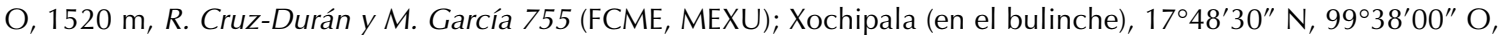
1050 m, C. Franco G. 10 (FCME); 3 km NO of Zumpango, $17^{\circ} 41^{\prime} \mathrm{N}, 99^{\circ} 32^{\prime} \mathrm{W}$, B. Hansen, J. Hansen y M. Nee 1501

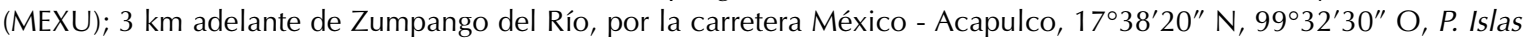

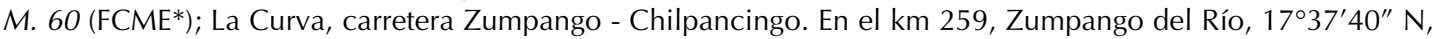

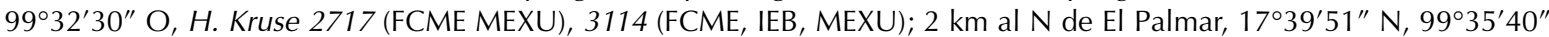

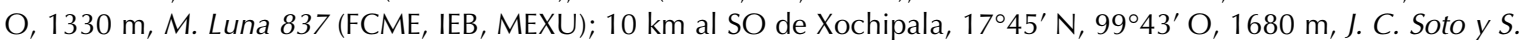
Aureoles 8244 (MEXU); 7 km al N de Zumpango del Río, 17²2' N, 99³3' O, 850 m, J. C. Soto y F. Solórzano 12666 (CICY, MEXU). General Heliodoro Castillo: 4 km al NE de Yextla, 17³8'24" N, 9955'43" O, 1450 m, J. Calónico S. 8241 (FCME). Huamuxtitlán: $1 \mathrm{~km}$ al NNE de Jilotepec, carreterea Palomas - Tlapa, 1758'19" N, 98 $28^{\prime} 44^{\prime \prime} \mathrm{N}, 1260 \mathrm{~m}$, E. Moreno G. y M. A. Monroy de la R. 735 (FCME). José Azueta: ca. $200 \mathrm{~m}$ al NE del caserío La Vainilla, camino al Calabazalito, $17^{\circ} 42^{\prime} \mathrm{N}, 101^{\circ} 31^{\prime} 30^{\prime \prime} \mathrm{O}, 250 \mathrm{~m}$, C. Gallardo, F. Lorea y A. Hanan 428 (FCME, IEB, MEXU); cañada situada

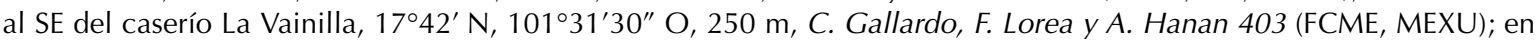

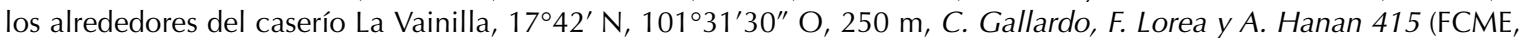
MEXU), C. Gallardo, F. Lorea y G. Ávila 496 (FCME, MEXU); lado NE del Cerro Viejo, Zihuatanejo, 17³9'30" N, 10132'13" O, 105 m, M. Gual 576 (FCME); 20 km al NNE de Zihuatanejo, camino Zihuatanejo - Ciudad Altamirano, $17^{\circ} 45^{\prime} 30^{\prime \prime} \mathrm{N}, 101^{\circ} 30^{\prime} 10^{\prime \prime} \mathrm{O}, 280 \mathrm{~m}$, F. Lorea 4035 (FCME); $3 \mathrm{~km}$ al NO de Vallecitos de Zaragoza, $17^{\circ} 57^{\prime} \mathrm{N}, 101^{\circ} 20^{\prime}$

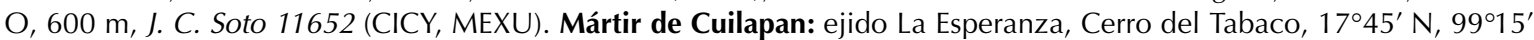
O, 1560 m, C. Teyuco 22 (FCME); La Esperanza, reserva campesina, 1745'35" N, 99¹4'58" O, N. Diego 7755 (FCME).

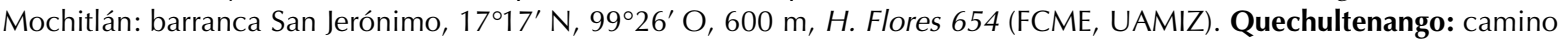

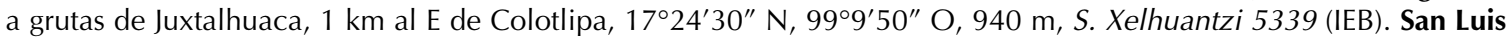
Acatlán: mojonera de Atotonilco, $3 \mathrm{~km}$ al NO de Atotonilco o a $11 \mathrm{~km}$ al NO de Horcasitas, camino San Luis Acatlán Pascala del Oro, $16^{\circ} 55^{\prime} \mathrm{N}, 98^{\circ} 51^{\prime} \mathrm{O}, 800$ m, E. Martínez, J. Victorino y B. Morales de Jesús 3537 (MEXU).

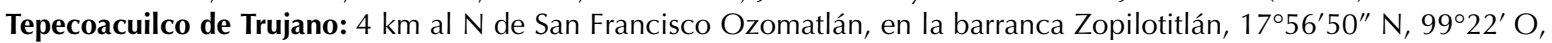
$1260 \mathrm{~m}$, A. Vargas-Pérez 322 (FCME, UAMIZ). Tixtla de Guerrero: hondonada a $2.5 \mathrm{~km}$ al O de Tixtla, 17³ $33^{\prime} 10^{\prime \prime} \mathrm{N}$, 99²5' O, 1600 m, G. Bonilla-González 12 (FCME, UAMIZ); al S de la presa El Molino, ca. 6 km al SO de Tixtla,

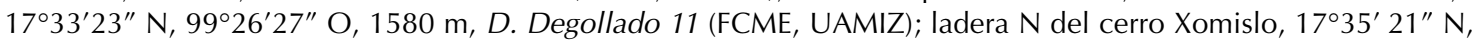
99²5'40" O, 1640 m, E. Velázquez M. 1423 (FCME, UAMIZ); al S de la presa El Molino, ca. 6 km al SO de Tixtla,

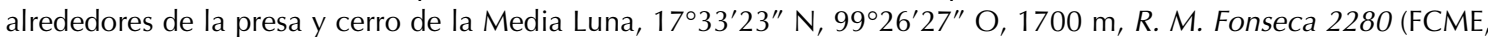
UAMIZ); orilla O de la presa El Molino, entre la antena y la cortina de la presa, $17^{\circ} 33^{\prime} 23^{\prime \prime} \mathrm{N}, 9^{\circ} 26^{\prime} 28^{\prime \prime} \mathrm{O}, 1600 \mathrm{~m}, R$.

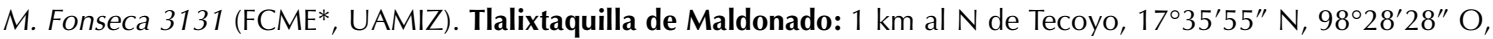
1000 m, J. Calónico S. 1718 (FCME), 1735 (FCME). Xochihuehuetlán: barranca Zacazonapa, 1.25 km al NNE de Jilotepec, $17^{\circ} 58^{\prime} 30^{\prime \prime} \mathrm{N}, 98^{\circ} 28^{\prime} 45^{\prime \prime} \mathrm{O}, 1260 \mathrm{~m}$, E. Moreno G. et al. 860 (FCME); cañada Axiococa, lado NNE del cerro Xilotzin, $17^{\circ} 59^{\prime} \mathrm{N}, 9^{\circ} 29^{\prime} 29^{\prime \prime} \mathrm{O}, 1340 \mathrm{~m}$, E. Moreno G. et al. 893 (FCME).

78. Tillandsia supermexicana Matuda, Cact.Suc.Mex. 22:21-22, f. 12. 1977.

Figura 21

DISTRIBUCIÓN: Endémica de México.

ALTITUD: 500-2000 m.

EJEMPLARES EXAMINADOS: Atlixtac: $1.86 \mathrm{~km}$ al S de Tozoyapan, 17³4'48”N $9^{\circ} 9^{\circ} 01^{\prime} 06^{\prime \prime} \mathrm{O}, 1550 \mathrm{~m}, \mathrm{C}$. A. Hernández A. 29 (FCME*); unterhalb microondas Pozo Largo - Petatlán, 17³6' $1^{\prime \prime}$ N, 99²' W, 1950 m, H. y L. Hromadnik 16046 (WU). General Heliodoro Castillo: 4 km al NE de Yextla, 17³8'24" N, 9955'43" O, 1450 m, J. Calónico S. 8245 (FCME*); Bosque de Hierbabuena, en la sierra costera, $17^{\circ} 31^{\prime} \mathrm{N}, 9^{\circ} 58^{\prime} \mathrm{O}, 1900 \mathrm{~m}$, E. Matuda 38641 (CODAGEM,

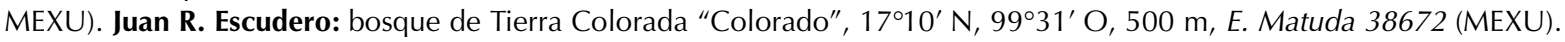

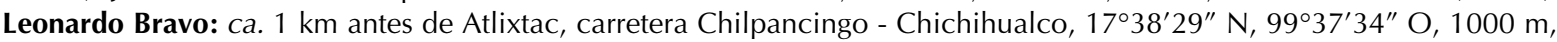
J. Calónico S. 7944 (FCME*); 0.5 km al O de La Escalera, 17²43'41" N, 9951'04" O, 1450 m, J. Calónico S. 8722 (FCME*). Zitlala: Topiltepec, 17³9'1" N, 99¹3'2" O, 1500 m, N. Diego y B. González H. 7302 (FCME*), B. Toazinque 7 (FCME*).

OTROS REGISTROS: Atlixtac: in declivis saxosis viae inter Pozo Largo et Petatlán17º36'1” N, 99²' O, 2000 m, H. y L. Hromadnik 14127 [Bromelie 1993/2].

79. Tillandsia taxcoensis Ehlers, Bromelie 1996:26-27, figs. 1996. DISTRIBUCIÓN: Endémica de México.

ALTITUD: $1800-1900 \mathrm{~m}$.

EJEMPLARES EXAMINADOS: Taxco de Alarcón: near the city of Taxco, along the road to Ixcateopan, $18^{\circ} 34^{\prime} \mathrm{N}, 99^{\circ} 37^{\prime}$ W, 1800-1900 m, K. Ehlers y R. Ehlers EM892602 (UAMIZ). 
80. Tillandsia trauneri L.Hrom., Bromelie 1994: 50-53, figs. 1994.

Figura 21

DISTRIBUCIÓN: Endémica de México.

ALTITUD: ca. $1100 \mathrm{~m}$.

EJEMPLARES EXAMINADOS: Coyuca de Catalán: ca. km 65, von Arroyo del Viejo, 1100 m, Hromadnik y Hromadnik 14033 (WU); SW of Ciudad Altamirano, at perpendicular rockface near Arroyo del Viejo, 1100 m, Hromadnik y Hromadnik 15076 (WU).

81. Tillandsia tricolor Schltdl. et Cham., Linnaea 6:54. 1831.

Figura 22 DISTRIBUCIÓN: México a Panamá.

ALTITUD: $1050 \mathrm{~m}$.

EJEMPLARES EXAMINADOS: Chilpancingo de los Bravo: Agua de Obispo, 17²19’ N, 99²9’ O, 1050 m, N. Diego 3998 (FCME*).

82. Tillandsia usneoides (L.) L., Sp.PI. ed. 2. 411. 1762.

Figura 21

DISTRIBUCIÓN: Estados Unidos a Argentina.

ALTITUD: 1090-2300 m.

EJEMPLARES EXAMINADOS: Atoyac de Álvarez: en El Ranchito, 13 km al NE de Paraíso, 17²3’ N, 100¹2’ O, 1090 m, J. C. Soto y S. Román 10074 (MEXU). Chilapa de Álvarez: $5.5 \mathrm{~km}$ de el NE de Hueycaltenango, sobre el camino Alcosacán - Hueycaltenango, 17²8'30" N, 99²'30" O, 2010 m, G. Lozano V. 411 (FCME*). Chilpancingo de los Bravo: El Chayotillo, limita con ejido Chautipa, parque estatal Omiltemi, 17³3'30" N, 9940' O, N. Diego y N. Arroyo H. 6832

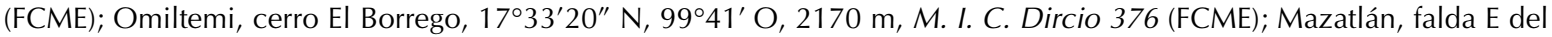
cerro Alquitrán, $17^{\circ} 26^{\prime} 1^{\prime \prime} \mathrm{N}, 9^{\circ} 27^{\prime} 54^{\prime \prime} \mathrm{O}, 2175 \mathrm{~m}$, H. Kruse 2318 (FCME, MEXU); cerro Cabeza de Toro, 17 $7^{\circ} 18^{\prime} \mathrm{N}$,

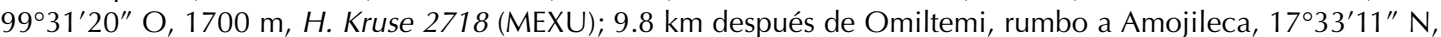

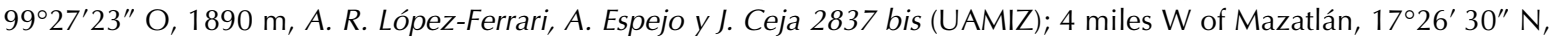

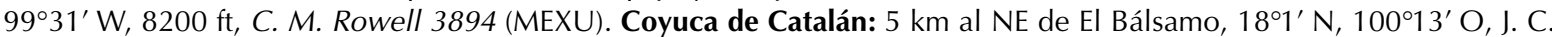
Soto, A. Román de Soto, y F. Soto 9723 (MEXU). Eduardo Neri: 2 km adelante de La Laguna, $17^{\circ} 44^{\prime} \mathrm{N}, 9^{\circ} 44^{\prime} 01^{\prime \prime} \mathrm{O}$,

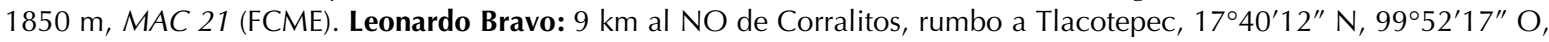
2300 m, J. Calónico 7386 (FCME); 1 km al N de El Pasclar, 17³5’ 59" N, 9948’01" O, 2090 m, J. Calónico 8216 (FCME); Tres Caminos, 3.94 km al SO, rumbo a Jaleaca de Catalán, 17³2' 30" N, 9953'56" O, 1700 m, J. Calónico 8792 (MEXU); 4.76 km al SE de Filo de Caballo, 17³6'56" N, 9948'47" O, 2250 m, J. Calónico 8973 (FCME); 1.18 km

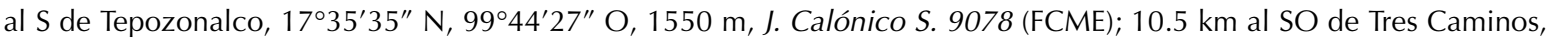
$17^{\circ} 32^{\prime} 30^{\prime \prime} \mathrm{N}, 9^{\circ} 54^{\prime} 40^{\prime \prime}$ O, 1820 m, J. Calónico S. y Y. Pascacio M. 10011 (FCME); Los Morros, a 8 km de Filo de

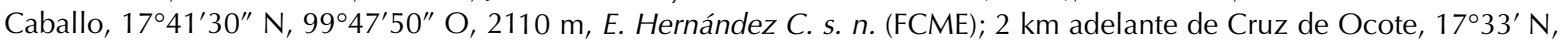
9954' O, 1890 m, G. Lozano 170 (FCME, MEXU); 59 km sobre la desviación a Filo de Caballo, carretera Iguala Chilpancingo, 1744' N, 9948' O, 2165 m, M. Flores, A. Martínez, S. Camargo y Santana 996 bis (UAMIZ); Campo de Aviación, cerca de Camotla, 170 $40^{\prime} \mathrm{N}, 9^{\circ} 48^{\prime} \mathrm{O}, 2250 \mathrm{~m}$, J. Rzedowski 16370 (MEXU). Malinaltepec: Malinaltepec, $17^{\circ} 15^{\prime} \mathrm{N}, 98^{\circ} 40^{\prime} \mathrm{O}, 1700 \mathrm{~m}$, I. Wagenbreth 72 (MEXU). Tlacoapa: El Tejocote (camino a Tlacoapa) a $54 \mathrm{~km}$ de Tlatlauquitepec, $17^{\circ} 18^{\prime} 30^{\prime \prime} \mathrm{N}, 98^{\circ} 46^{\prime} 55^{\prime \prime} \mathrm{O}, 2045$ m, J. Almazán 97 (FCME*).

83. Tillandsia violacea Baker, J.Bot. 25:279. 1887.

Figura 22

DISTRIBUCIÓN: Endémica de México.

ALTITUD: $1700-3100 \mathrm{~m}$.

EJEMPLARES EXAMINADOS: Atoyac de Álvarez: ca. $3 \mathrm{~km}$ después de Puerto del Gallo, rumbo a Filo de Caballo, $17^{\circ} 28^{\prime} 14^{\prime \prime} \mathrm{N}, 100^{\circ} 10^{\prime} 22^{\prime \prime} \mathrm{O}, 2500 \mathrm{~m}$, E. Domínguez-Licona 566 (FCME); $2.2 \mathrm{~km}$ adelante de Puerto del Galllo, rumbo a Filo de Caballo, 17²7'53" N, 10009'56" O, 2570 m, E. Domínguez-Licona 1085 (FCME); al SE de Puerto del Gallo,

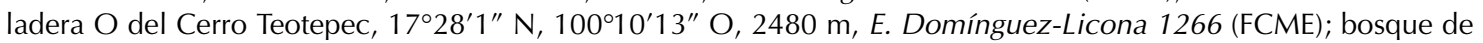
Atoyac, $17^{\circ} 12^{\prime} \mathrm{N}, 100^{\circ} 20^{\prime} \mathrm{O}, 1700 \mathrm{~m}$, E. Matuda 38702 (MEXU); $6 \mathrm{~km}$ después de Puerto del Gallo, hacia el Cerro

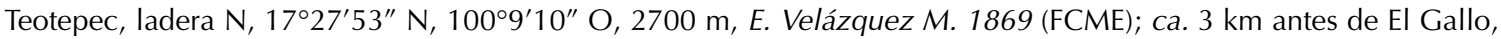

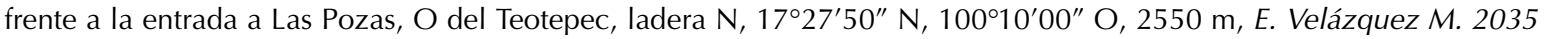
(FCME). General Heliodoro Castillo: Agua Fría, $17^{\circ} 27^{\prime} \mathrm{N}, 100^{\circ} 4^{\prime} \mathrm{O} ; 2500 \mathrm{~m}, \mathrm{~N}$. Diego, B. Ludlow y A. Acosta 7712 (FCME); Teotepec, ladera S, 17²6'59" N, 1004'18" O, 2900 m, N. Diego, A. Acosta y G. Fernández 8663 (FCME); a 5

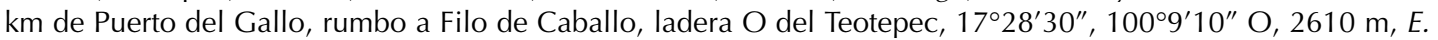
Domínguez-Licona 1152 (FCME); $5 \mathrm{~km}$ al SO de puerto El Jilguero, camino a Puerto del Gallo, 17 $27^{\prime} 20^{\prime \prime} \mathrm{N}, 100^{\circ} 2^{\prime} \mathrm{O}$,

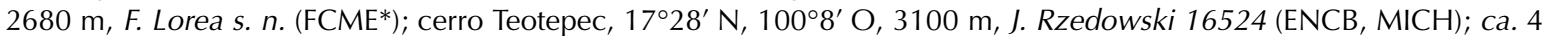
km después de Puerto del Gallo, hacia el cerro Teotepec, 17²7'51" N, 1009'39” O, 2610 m, E. Velázquez 1913a 
(FCME). Leonardo Bravo: 4 km al S de Carrizal de Bravo, 17³5’07" N, 9950’49" O, J. Calónico S. 8295 (FCME*); 7.56 km al S de Filo de Caballo, 17³3'59" N, 9952'50" O, J. Calónico S. 8532 (FCME*); 8 km al S de Carrizal de Bravo,

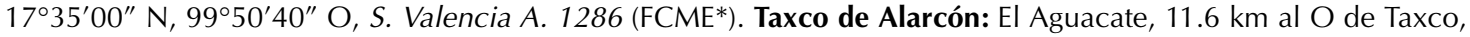

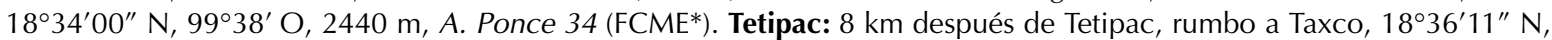
99³7'49" O, 2070 m, A. Espejo, A. R. López-Ferrari, J. Ceja, Mendoza e I. Ramírez 6181 (UAMIZ).

84. Tillandsia xerographica Rohweder, Senckenbergiana 34:113, f. 8-11, t. 1, f. 2. 1953.

Figura 22 DISTRIBUCIÓN: México a El Salvador y Guatemala.

ALTITUD: 600-672 m.

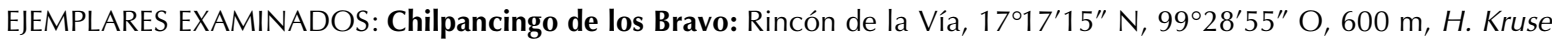
3118 (CODAGEM, MEXU), C. S. Gardner 1212 sub H. Luther s. n. (SEL).

\section{Ursulaea Read et Baensch}

85. Ursulaea tuitensis (Magaña et E.J.Lott) Read et Baensch, J.Bromeliad Soc. 44:209, f. 6. 1994.

Figura 23 DISTRIBUCIÓN: Endémica de México.

ALTITUD: $1500 \mathrm{~m}$.

EJEMPLARES EXAMINADOS: Zitlala: Topiltepec (reserva campesina), 1739' $1^{\prime \prime} \mathrm{N}, 9^{\circ} 13^{\prime} 2^{\prime \prime} \mathrm{O}, 1500 \mathrm{~m}$, B. Toazinque 7$A($ FCME*).

\section{VIRIDANTHA ESPEJO}

1. Infloresencia nidular, el pedúnculo ausente o más corto que las hojas. V. atroviridipetala

1. Inflorescencia pedunculada, el pedúnculo igualando o más largo que las hojas.

2. Espiga solitaria en el ápice de la inflorescencia. V. ignesiae

2. Espigas dos o más en el ápice de la inflorescencia..... V. plumosa

86. Viridantha atroviridipetala (Matuda) Espejo, Acta Bot.Mex. 60: 28-29, fig. 1, 2. 2002. DISTRIBUCIÓN: Endémica de México.

ALTITUD: 1050-2165 m.

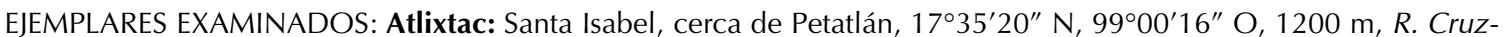
Durán et al. 5504 (FCME*); a 1.85 km al NO de Petatlán, 17³5’35" N, 9900’08” O, 1440 m, M. I. Limón G. 5504

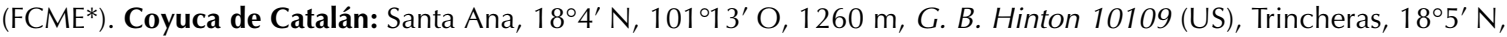
101¹6' O, G. B. Hinton et al. 10129 (GH, US). Eduardo Neri: Puerto de los Tepetates, 2 km al N de Amatitlán,

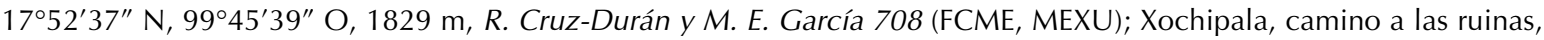
$17^{\circ} 49^{\prime} \mathrm{N}, 9^{\circ} 38^{\prime} 40^{\prime \prime} \mathrm{O}, 1050 \mathrm{~m}$, C. Franco G. 8 (FCME). Leonardo Bravo: 59 km sobre la desviación a Filo de Caballo, carretera Iguala - Chilpancingo, $17^{\circ} 41^{\prime} \mathrm{N}, 9^{\circ} 48^{\prime} \mathrm{O}, 2165 \mathrm{~m}, \mathrm{M}$. Flores, A. Martínez, S. Camargo y J. Santana 994 (UAMIZ). Tixtla de Guerrero: ladera N del cerro Xomislo, $17^{\circ} 35^{\prime} 21^{\prime \prime} \mathrm{N}, 99^{\circ} 25^{\prime} 40^{\prime \prime} \mathrm{O}, 1640 \mathrm{~m}$, E. Velázquez M. 1425 (FCME*, UAMIZ); mountain NE of Chilpancingo on road to Chilapa, $17^{\circ} 36^{\prime} \mathrm{N}, 99^{\circ} 20^{\prime} \mathrm{W}, 5800 \mathrm{ft}$, H. E. Moore y C. E. Wood 4631 (AA, MICH, US).

87. Viridantha ignesiae (Mez) Espejo, Acta Bot.Mex. 60: 29, fig. 2. 2002. DISTRIBUCIÓN: Endémica de México.

ALTITUD: $1500-1890 \mathrm{~m}$.

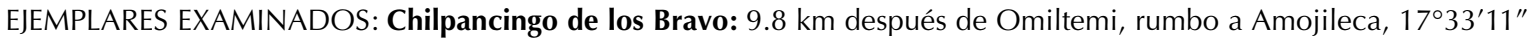
N, 99³7'23" O, 1890 m, A. R. López-Ferrari, A. Espejo y J. Ceja 2837 (UAMIZ). Coyuca de Catalán: Zacatlán, 185’ N, $101^{\circ} 7^{\prime} \mathrm{O}, 1500 \mathrm{~m}, \mathrm{G}$. B. Hinton et al. 10102 (GH, MEXU, MICH, MO, P, TEX).

88. Viridantha plumosa (Baker) Espejo, Acta Bot.Mex. 60:3, fig. 5. 2002.

Figura 23 DISTRIBUCIÓN: Endémica de México.

ALTITUD: $1450-2170 \mathrm{~m}$.

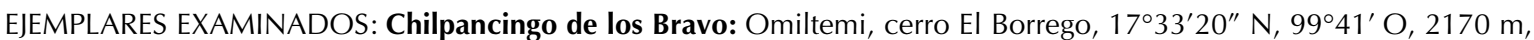

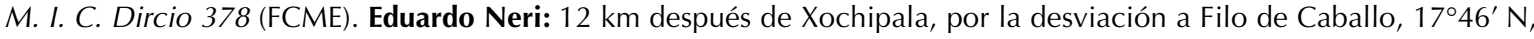


9942'20" O, 1810 m, MAC 14 (FCME*, UAMIZ); ca. 15 km después de Carrizal de Bravos, rumbo a Xochipala, 1744' $\mathrm{N}, 99^{\circ} 44^{\prime} \mathrm{O}, 2150 \mathrm{~m}$, A. R. López-Ferrari 363 (UAMIZ); $3 \mathrm{~km}$ adelante de El Mirabal, carretera Chilpancingo - Filo de

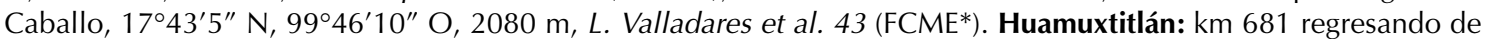

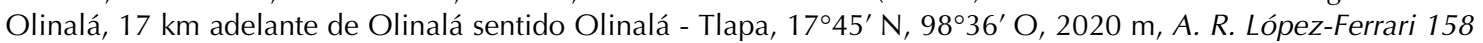

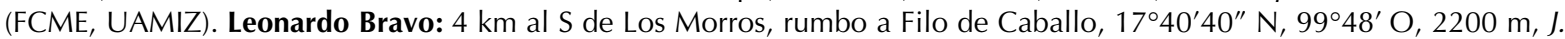
Calónico S. 3609 (FCME*); 1 km al NE de Campo de Aviación, 17³9'54" N, 9948'43" O, 2150 m, J. Calónico S. 4628 (FCME*), R. Cruz-Durán et al. 1457 (FCME*); 2 km al E de Campo de Aviación, 17³9'55" N, 9948’34" O, 2150 m, J. Calónico S. 8030 (FCME*); camino a La Felicidad, 4.55 km al SE de Filo de Caballo, 17³4'50" N, 9949’11" O, 2000 m, J. Calónico S. 8364 (FCME*); $21 \mathrm{~km}$ al O de Chilpancingo por la carretera a Chichihualco al final de una cañada (a 4

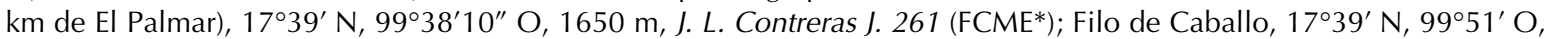
2200 m, N. Diego s. n. (FCME), C. Franco 18 (FCME, MEXU), J. C. Soto y F. Solórzano 12681 (MEXU); 59 km sobre la

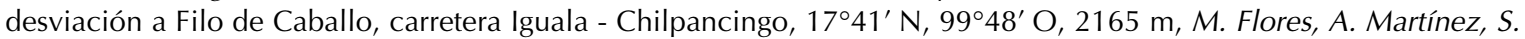
Camargo y J. Santana 995 (UAMIZ); 26 km al O de Xochipala, camino a Filo de Caballo, 17³9’ N, 9949’ O, 2060 m, E. Martínez 756 (MEXU, MO); Campo de Aviación, cerca de Camotla, 17²40’ N, 99²48' O, 2250 m, J. Rzedowski 16361 (ENCB, MICH). Malinaltepec: Malinaltepec, $17^{\circ} 15^{\prime} \mathrm{N}, 98^{\circ} 40^{\prime} \mathrm{O}, 1800 \mathrm{~m}$, l. Wagenbreth 522 (MEXU, MO). Metlatónoc:

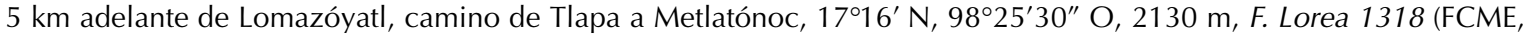

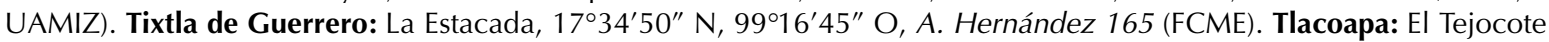

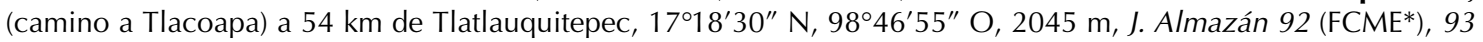
(FCME*).

\section{Taxa excluidos o dudosos}

Aechemea bracteata (Sw.) Griseb. var. pacifica Beutelsp.: Los ejemplares de A. bracteata procedentes de Guerrero y en general de toda la costa pacífica han sido ubicados en la supuesta variedad pacifica. Sin embargo, la revisión de cerca de 400 ejemplares recolectados a lo largo de todo el país, nos llevó a concluir que no es posible separar variedades, ya que no hay caracteres ni morfológicos ni ecológicos constantes que permitan diferenciarlas con claridad. Por esta razón, para fines de este trabajo no consideramos variedades de esta especie.

Billbergia mexicana Mez: El tipo de esta especie procede de Agua de Correa en el municipio de La Unión de Isidoro Montes de Oca; sin embargo, consideramos a dicho taxon como un sinónimo de Billbergia pallidiflora Liebm.

Hechtia glomerata Zucc.: Los especímenes citados por Smith y Downs (1974) como pertenecientes a esta especie pertenecen en realidad a $\boldsymbol{H}$. pumila Burt-Utley y Utley (Burt-Utley y Utley, 1988: 281).

Hechtia stenopetala Klotzsch.: El material procedente de Guerrero determinado con este nombre pertenece en realidad a H. melanocarpa (Burt-Utley y Utley, 1988: 282). H. stenopetala es endémica de Veracruz.

Pitcairnia densiflora Brongn. ex Lem. y Pitcairnia ochroleuca (K.Koch et Bouché) Baker: El material de Guerrero identificado con estos nombres pertenece a $\boldsymbol{P}$. imbricata (Brongn.) Regel.

Pitcairnia flexuosa L.B.Sm.: El material citado por Smith y Downs (1974) corresponde en realidad a P. oaxacana. No hemos visto, hasta ahora, ningún ejemplar de esta especie procedente del estado de Guerrero. Sin embargo, hay registros de la misma en los municipios vecinos de Tlatlaya (Estado de México) y Huetamo, Tiquicheo de Nicolás Romero y Tuzantla (Michoacán), por lo que posteriores exploraciones podrían resultar en su descubrimiento en el estado.

Pitcairnia puberula Mez et Donn.Sm.: Los ejemplares procedentes de Guerrero citados por Smith y Downs (1974) como representantes de esta especie, corresponden en realidad a $\boldsymbol{P}$. karwinskyana Schult. et Schult.f. P. puberula sólo se conoce con certeza de Guatemala.

Pitcairnia lanosisepala Matuda. Los ejemplares de Guerrero, identificados con este nombre corresponden en realidad a $P$. modesta.

Tillandsia argentea Griseb.: Esta especie, como lo señala Till (1992) habita únicamente en Las Antillas (Cuba y Jamaica). Las plantas mexicanas corresponden en realidad a $\mathbf{T}$. fuchsii Till.

Tillandsia bartramii Elliottt. Este taxon se conoce únicamente de la vertiente atlántica del país en los estados de Nuevo León, San Luis Potosí, Querétaro y Tamaulipas (Espejo et al., 2004). Las plantas de Guerrero identificadas o citadas con este nombre pertenecen en realidad a $\boldsymbol{T}$. pseudosetacea Ehlers. et Rauh.

Tillandsia chaetophylla Mez: Las plantas de Guerrero identificadas con este nombre, pertenecen en realidad a $T$. pentasticha Rauh et Wülfinghoff o a T. ortgiesiana E.Morren ex Mez.

Tillandsia dasyliriifolia Baker: Este taxon se conoce únicamente de la península de Yucatán (Espejo et al., 2004). Los ejemplares de Guerrero identificados con este nombre corresponden a T. makoyana Baker.

Tillandsia lautneri Ehlers: Los reportes de esta especie para Guerrero pertenecen en realidad a T. capitata Griseb. Tillandsia mooreana L.B.Sm.: Los especímenes provenientes del estado identificados con este nombre corresponden a $\boldsymbol{T}$. kalmbacheri Matuda. 
FLORA BROMELIOLÓGICA DE GUERRERO

Tillandsia paucifolia Baker: Esta especie se conoce únicamente de la vertiente atlántica del país (Espejo et al., 2004). El taxon que habita Guerrero y en general en la costa pacífica es $\boldsymbol{T}$. intermedia Mez.

Tillandsia roland-goselinii Mez: Las plantas procedentes de Guerrero identificadas con este nombre pertenecen en realidad a T. maritima Matuda.

Tillandsia roseospicata Matuda: No hemos podido ver ejemplares pertenecientes a este taxon procedentes de Guerrero. Sin embargo el tipo del mismo procede del vecino municipio de Zacualpan, en el Estado de México, por lo que podría a la larga encontrarse en la entidad.

Tillandsia streptophylla Scheidw. ex E.Morren.: No existe material de esta especie procedente de Guerrero, depositado en los herbarios revisados. Exploraciones más detalladas podrían quizás, demostrar su presencia en el estado.

Viridantha lepidosepala (L.B.Sm.) Espejo: Existe en MEXU un ejemplar de esta especie supuestamente recolectado por Matuda en Rincón de la Vía; sin embargo, dada la distribución más norteña de la especie, pensamos que los datos de dicho ejemplar no son confiables. 
Valeria Angélica Pulido-Esparza, Ana Rosa LóPeZ-Ferrari y Adolfo EsPejo-Serna

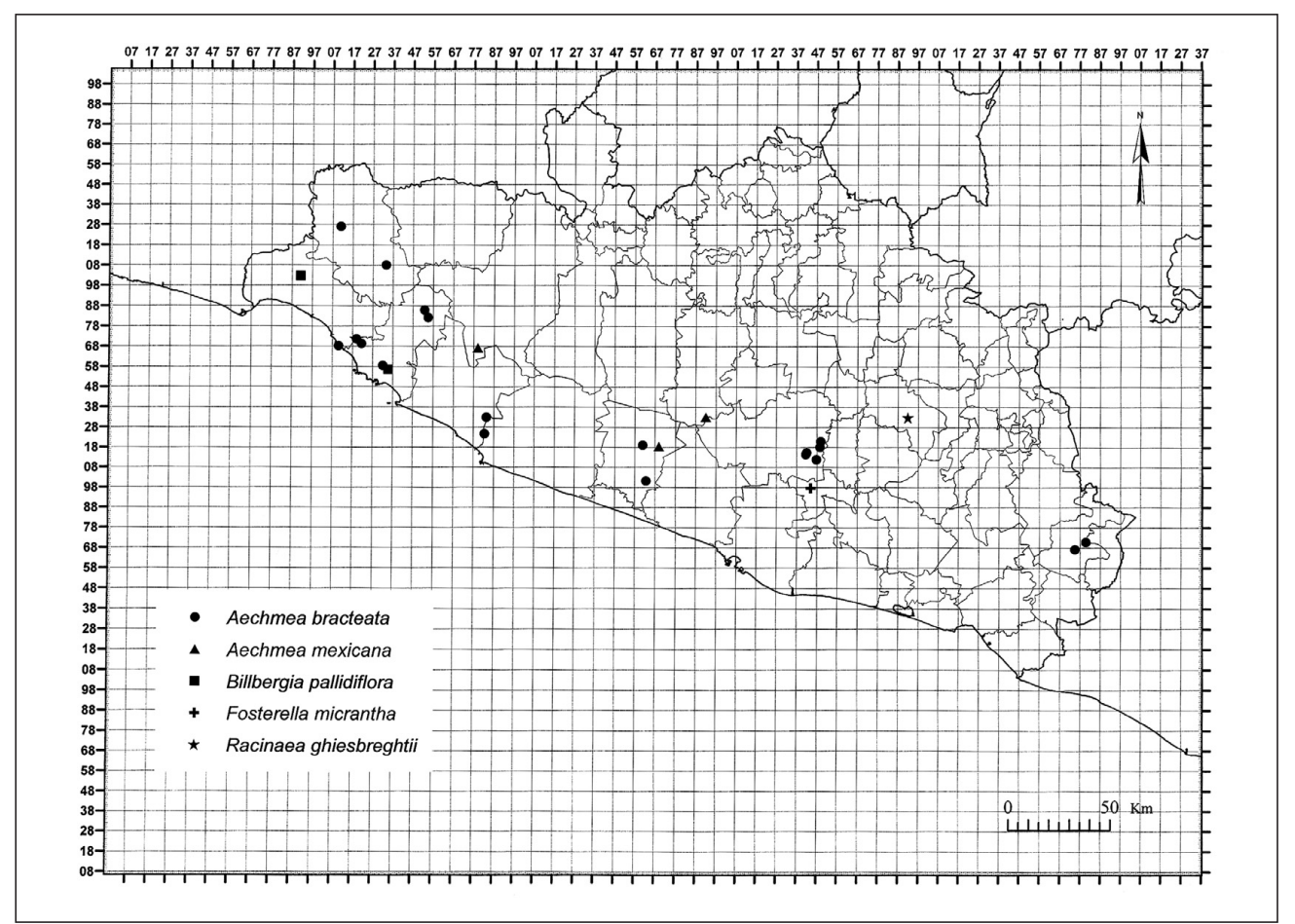

Figura 7. Distribución de las especies de Aechmea, Billbergia, Fosterella y Racinaea en Guerrero.

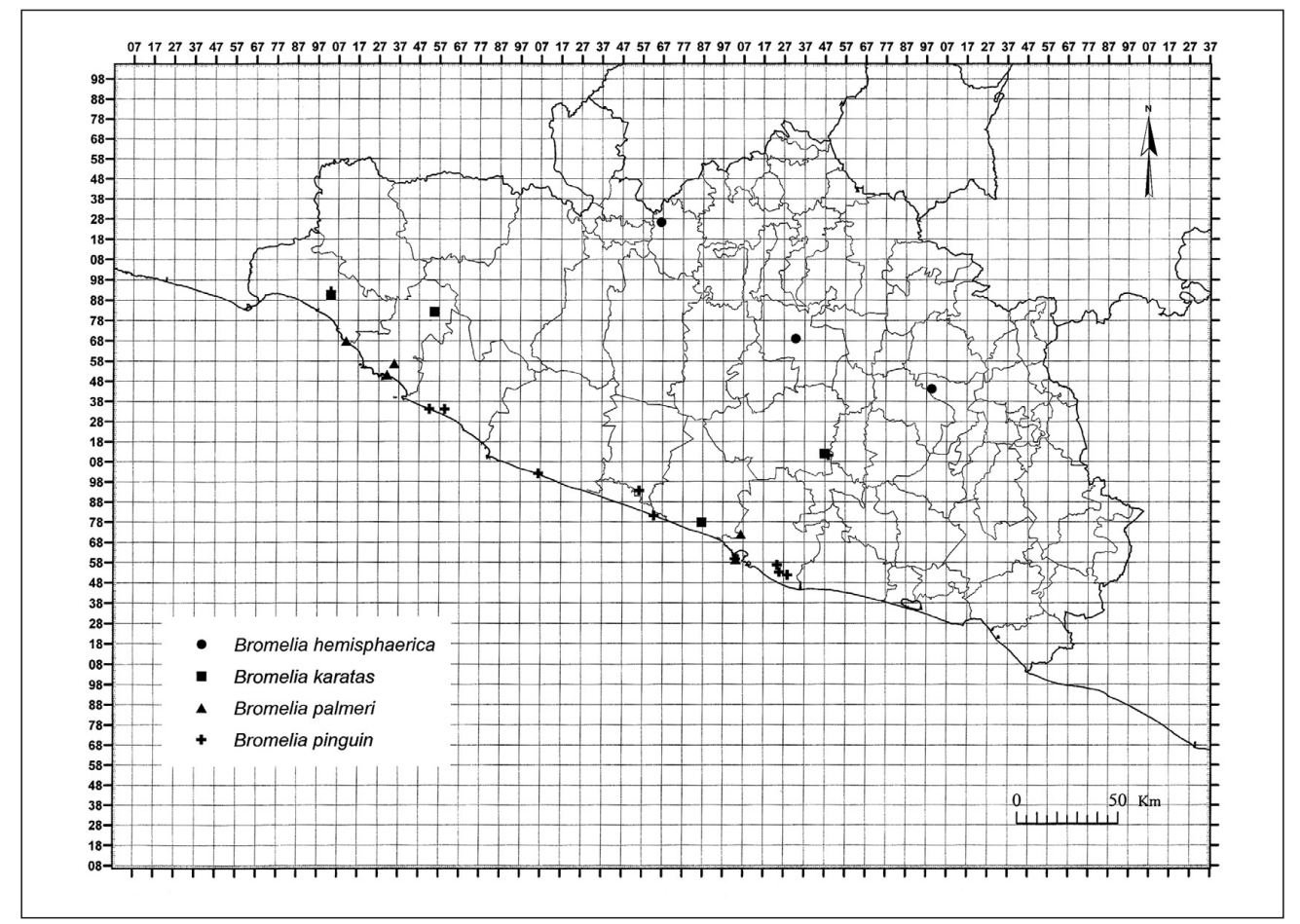

Figura 8. Distribución de las especies de Bromelia en Guerrero. 
FLORA BROMELIOLÓGICA DE GUERRERO

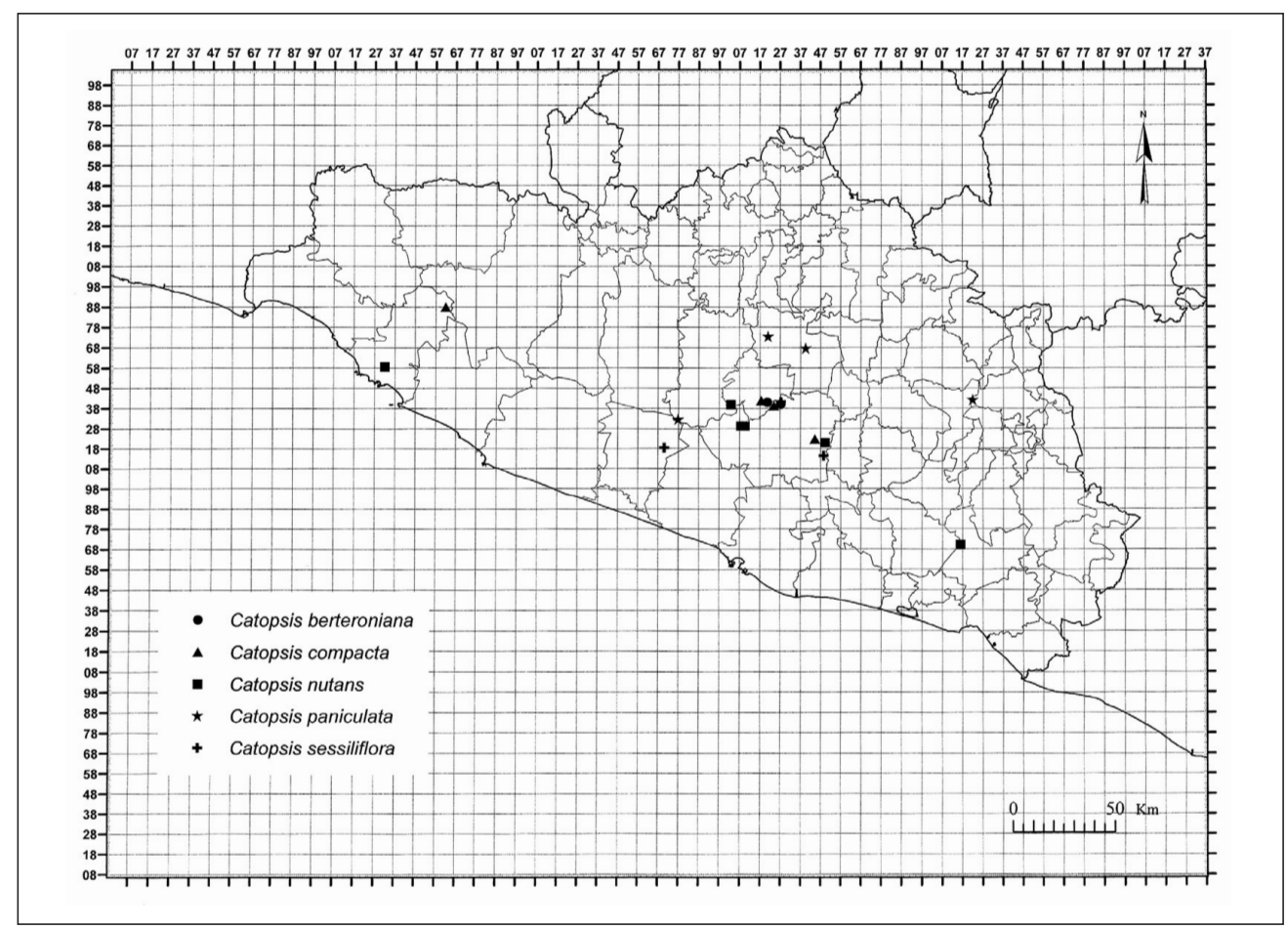

Figura 9. Distribución de las especies de Catopsis en Guerrero.

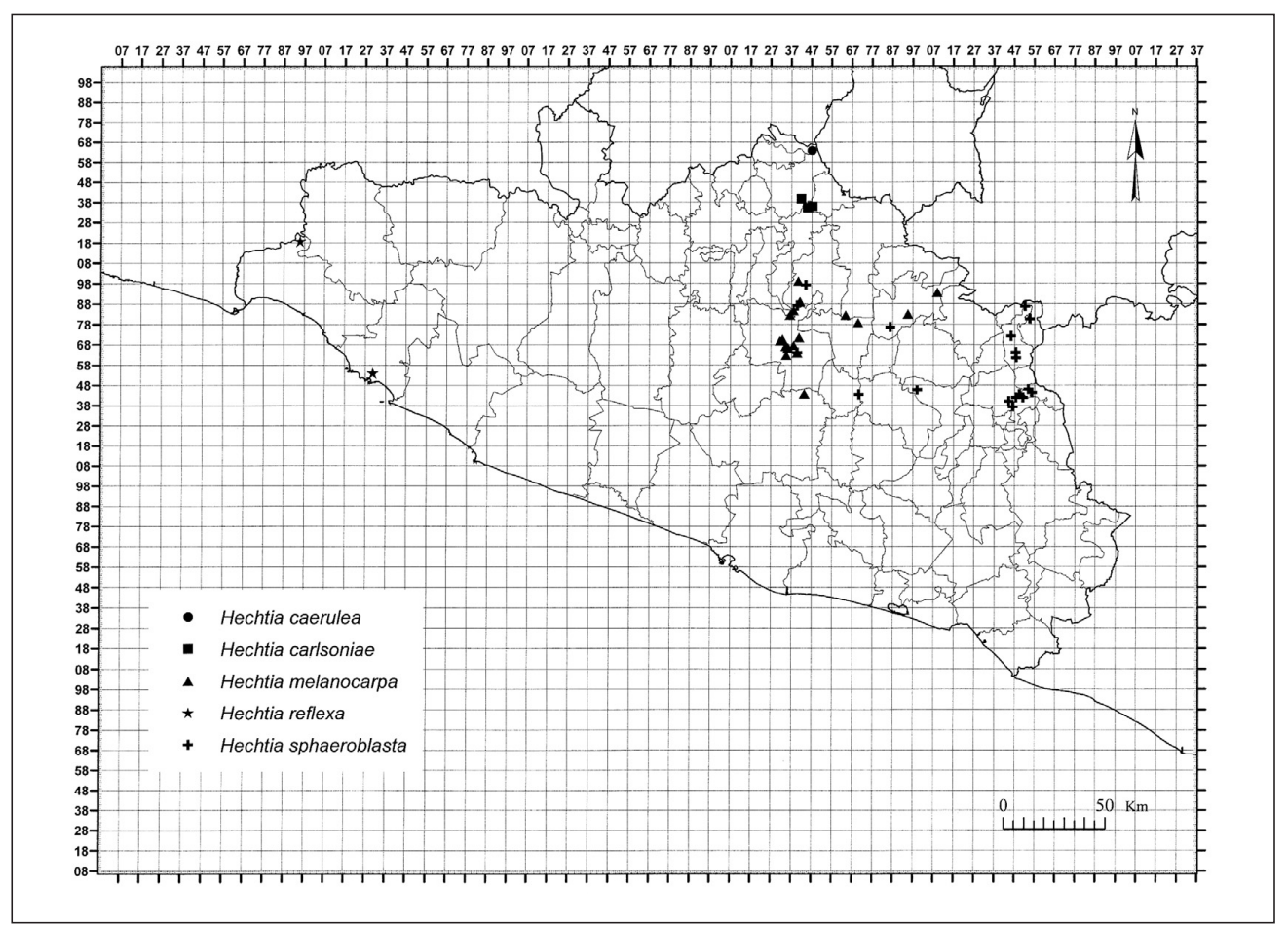

Figura 10. Distribución de algunas especies de Hechtia en Guerrero. 
Valeria Angélica Pulido-Esparza, Ana Rosa LóPeZ-Ferrari y Adolfo EsPejo-Serna

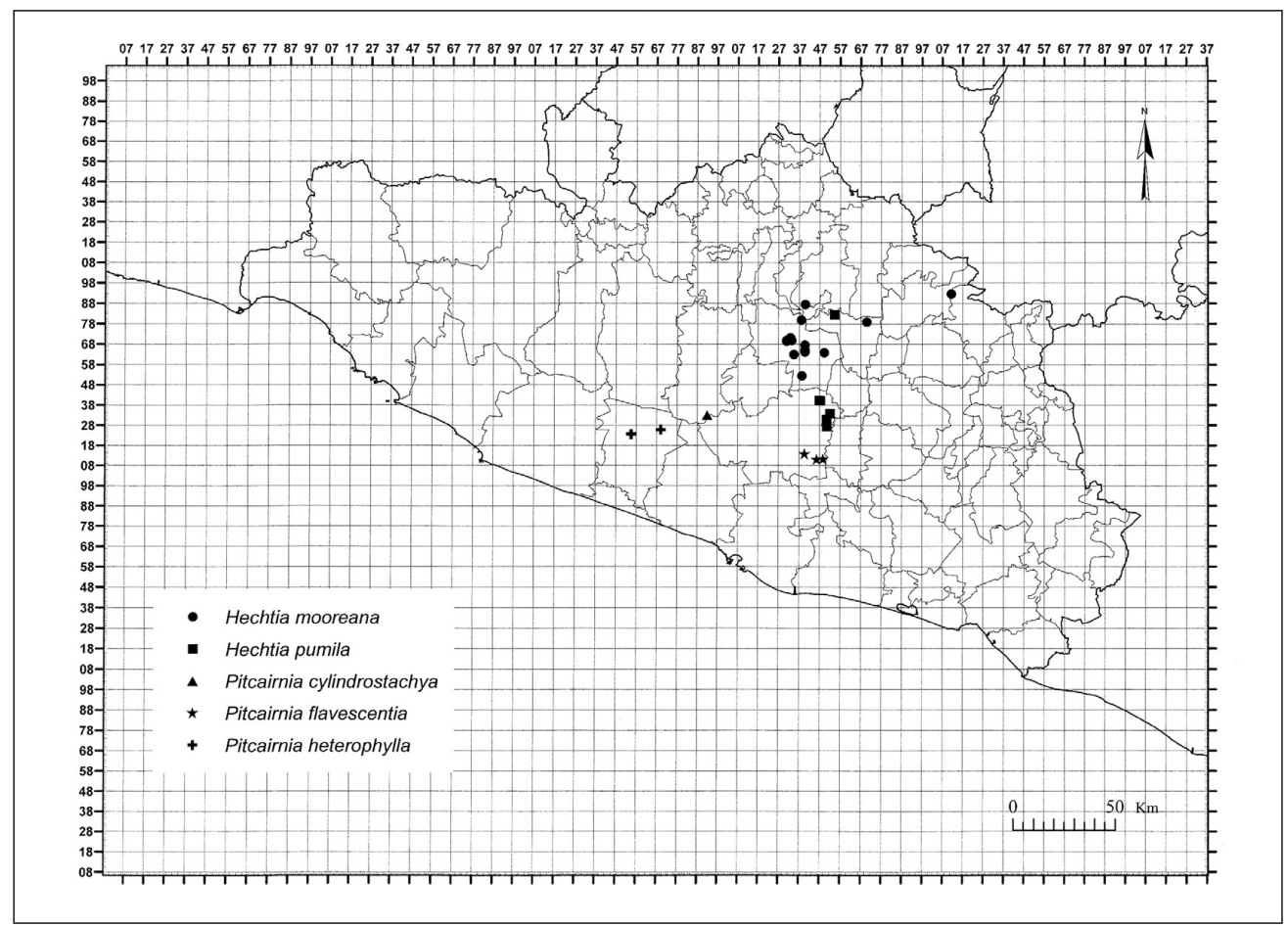

Figura 11. Distribución de algunas especies de Hechtia y Pitcairnia en Guerrero.

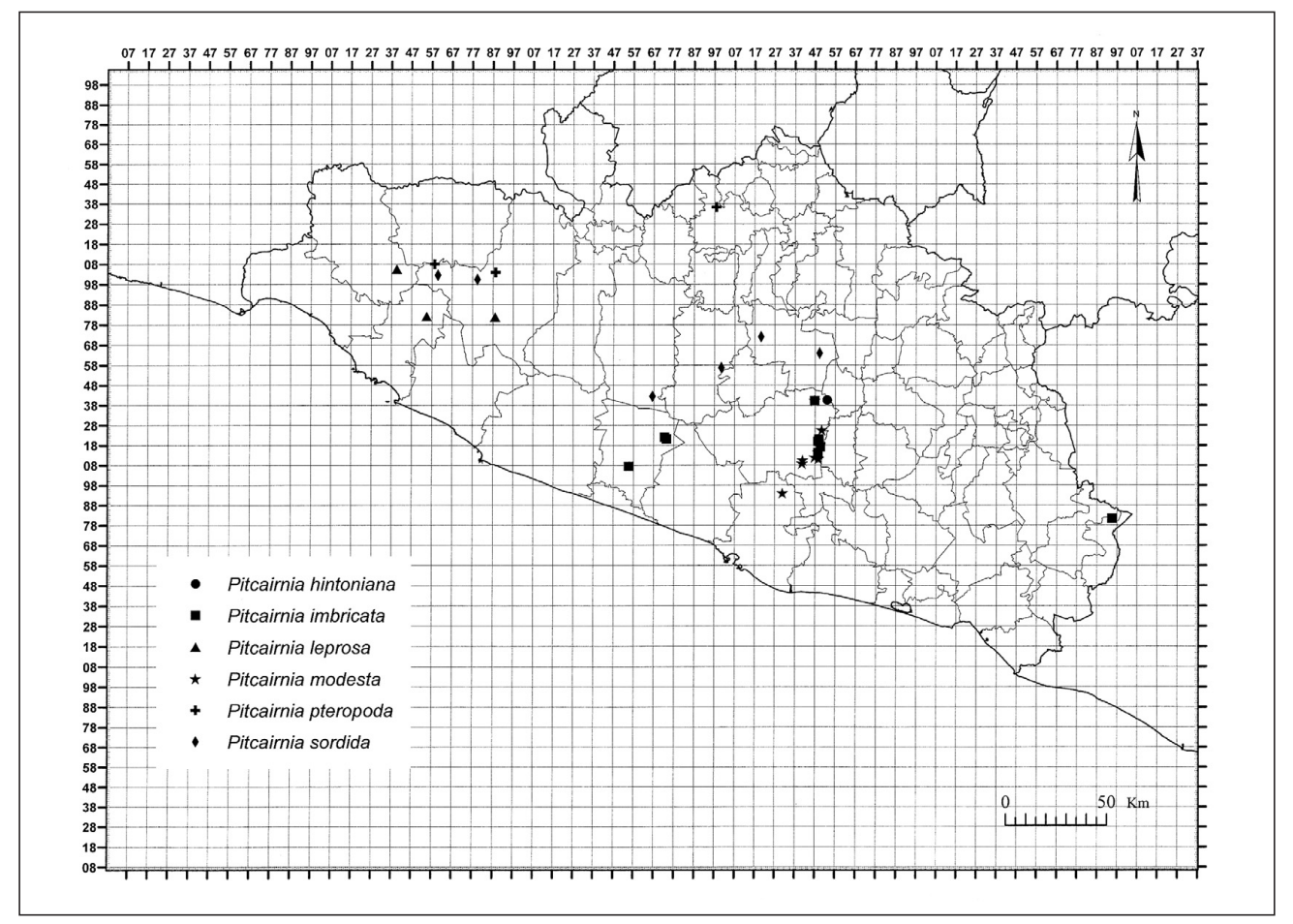

Figura 12. Distribución de algunas especies de Pitcairnia en Guerrero. 
FLORA BROMELIOLÓGICA DE GUERRERO

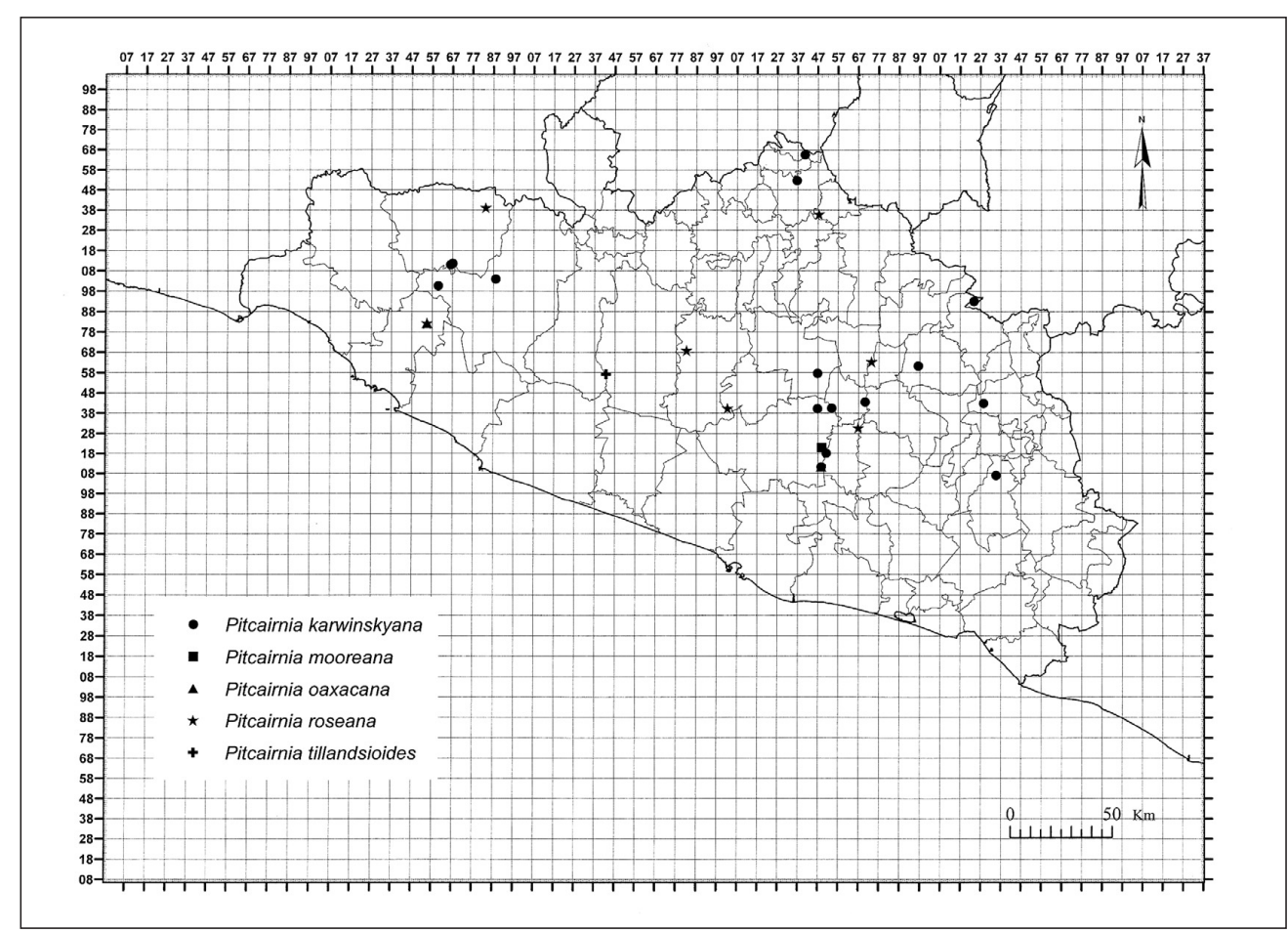

Figura 13. Distribución de algunas especies de Pitcairnia en Guerrero.

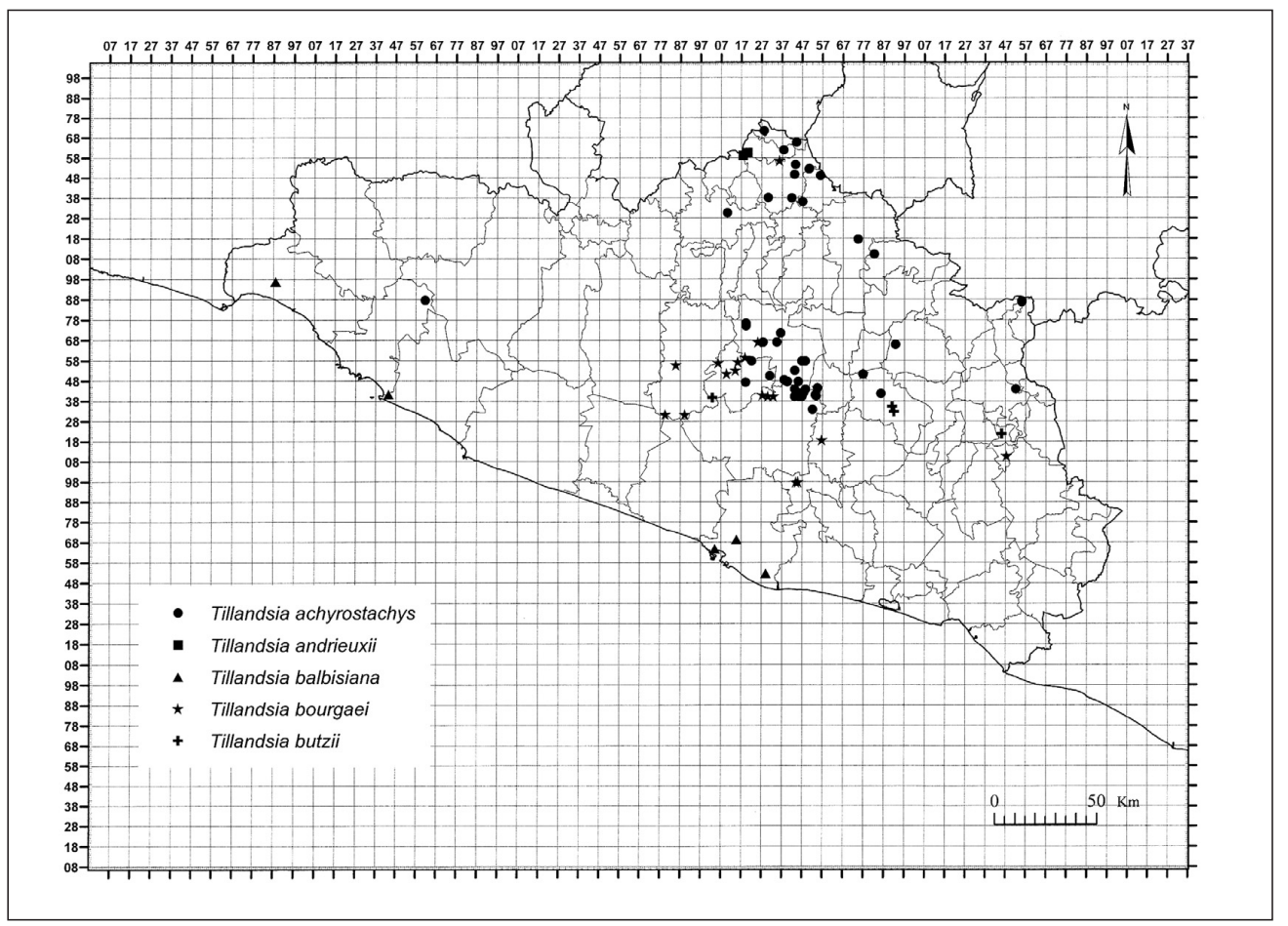

Figura 14. Distribución de algunas especies de Tillandsia en Guerrero. 
Valeria Angélica Pulido-Esparza, Ana Rosa LóPeZ-Ferrari y Adolfo EsPejo-Serna

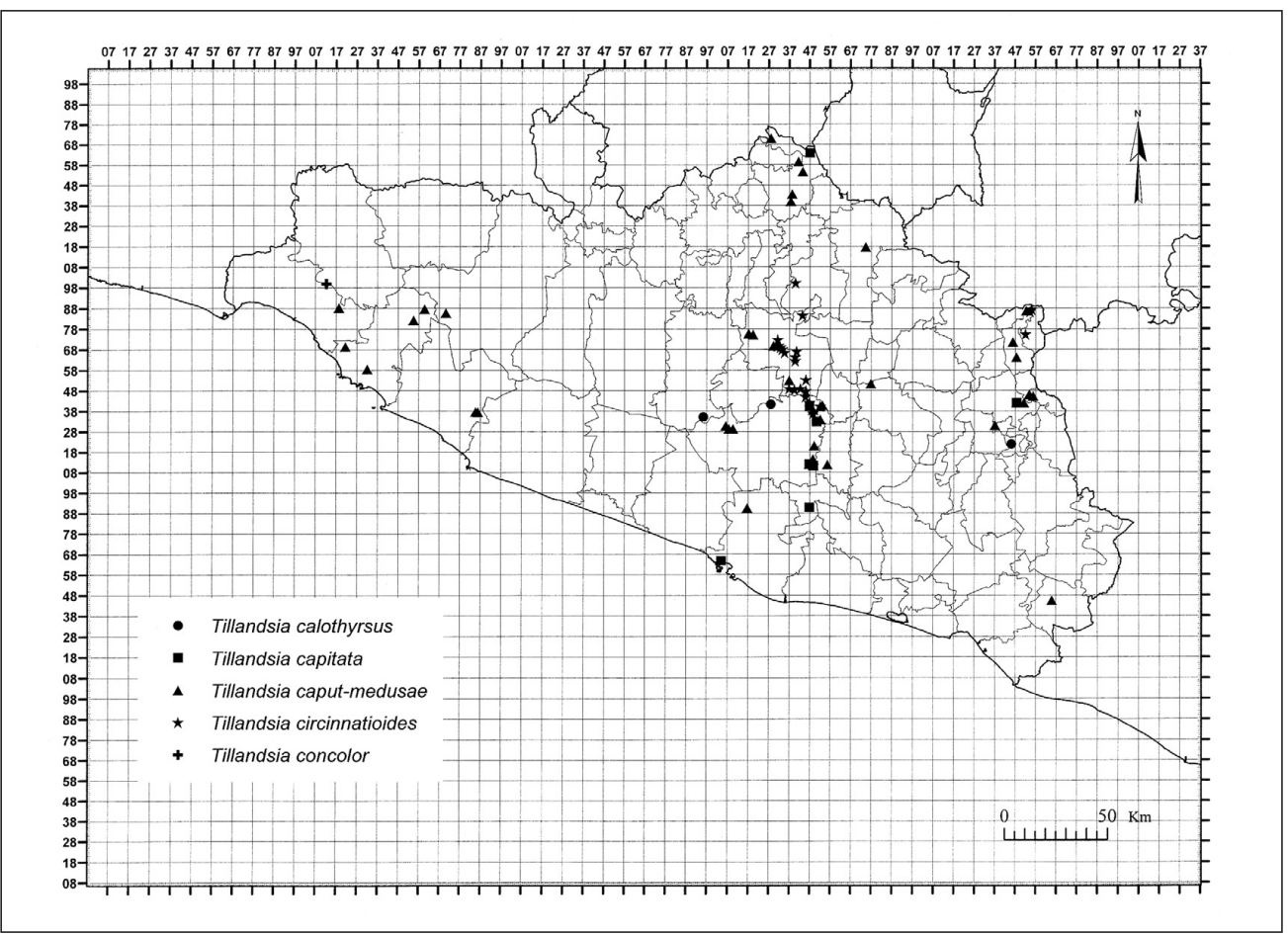

Figura 15. Distribución de algunas especies de Tillandsia en Guerrero.

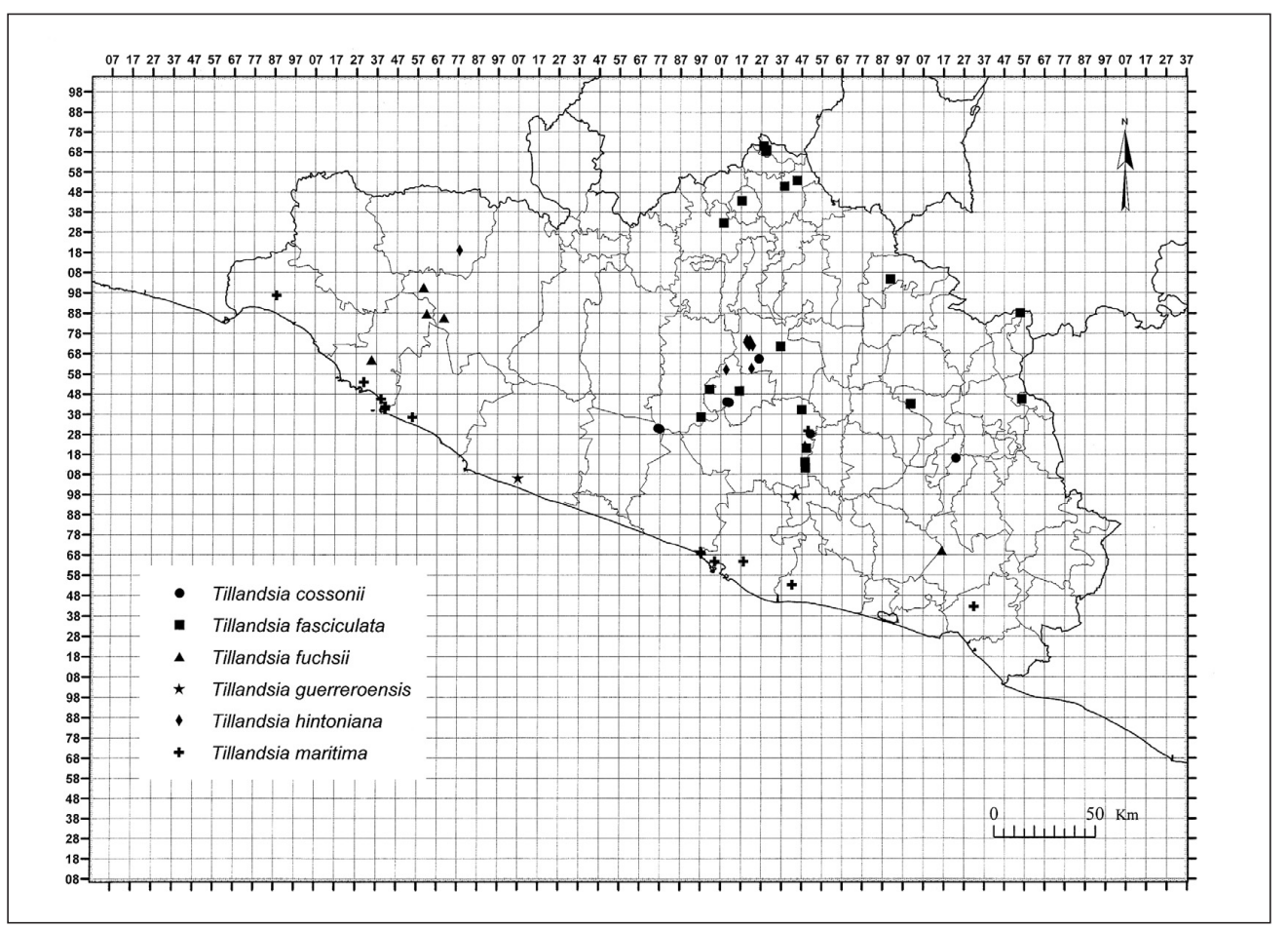

Figura 16. Distribución de algunas especies de Tillandsia en Guerrero. 
FLORA BROMELIOLÓGICA DE GUERRERO

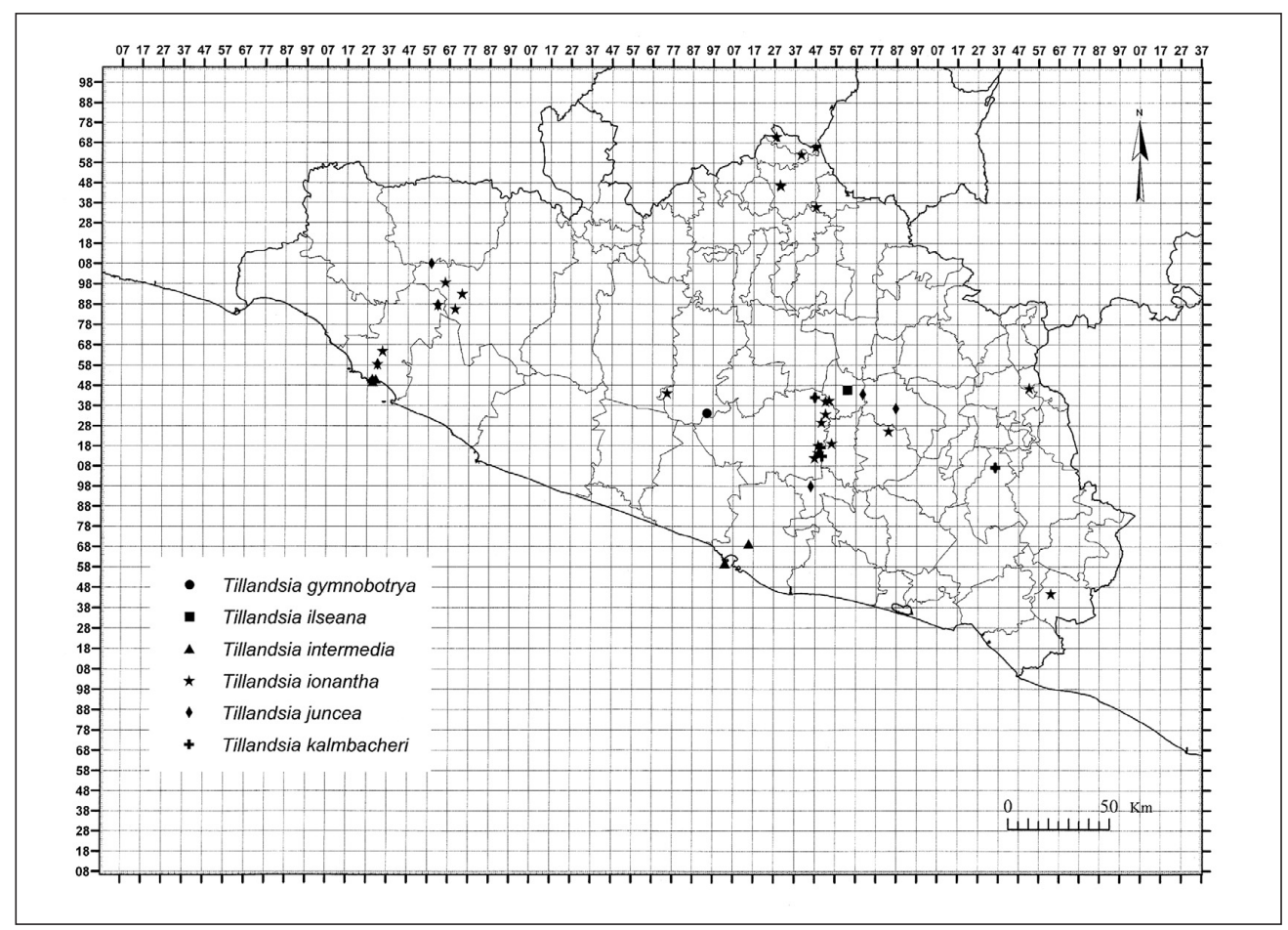

Figura 17. Distribución de algunas especies de Tillandsia en Guerrero.

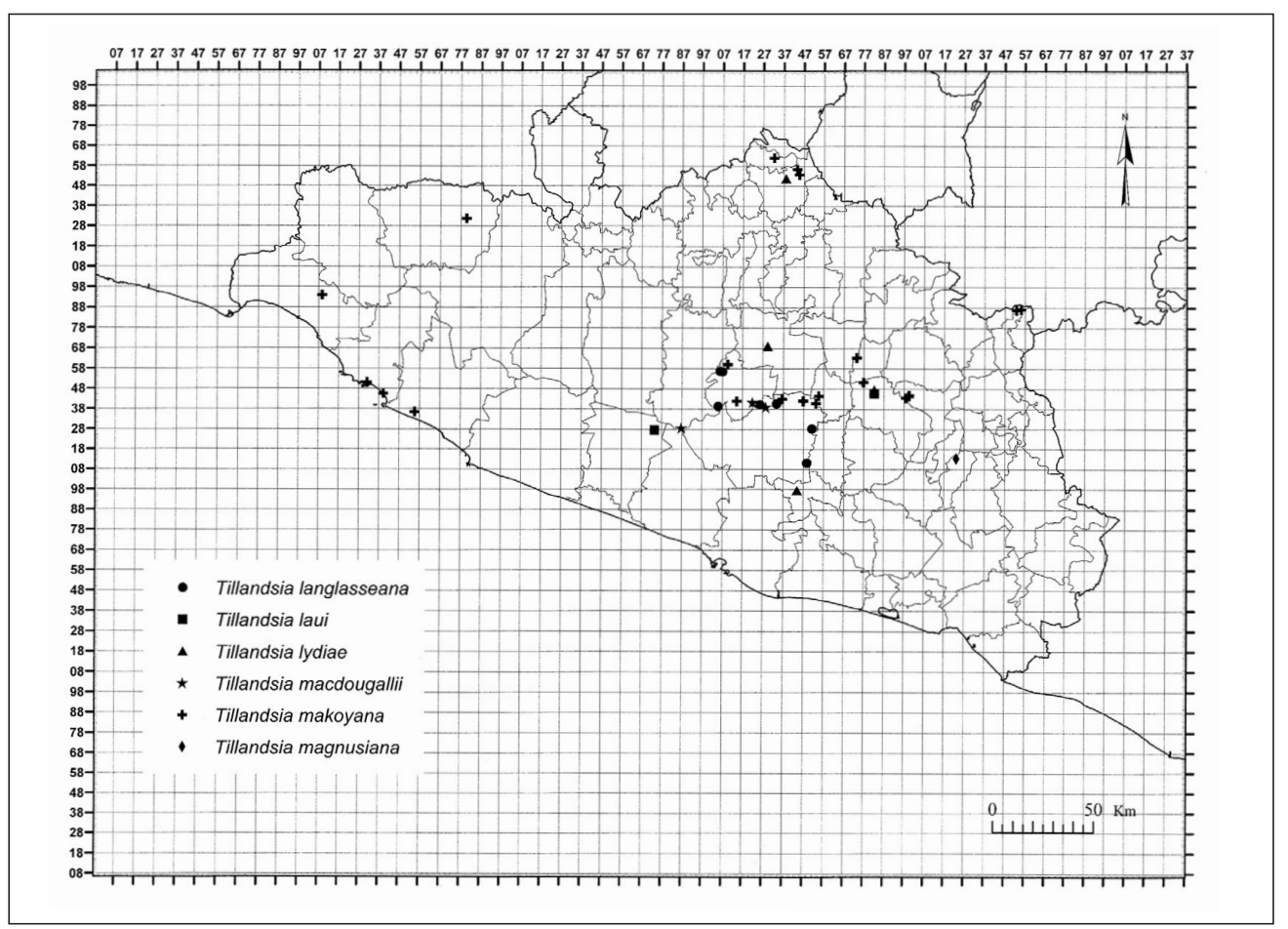

Figura 18. Distribución de algunas especies de Tillandsia en Guerrero. 
Valeria Angélica Pulido-Esparza, Ana Rosa LóPez-Ferrari y Adolfo EsPejo-Serna

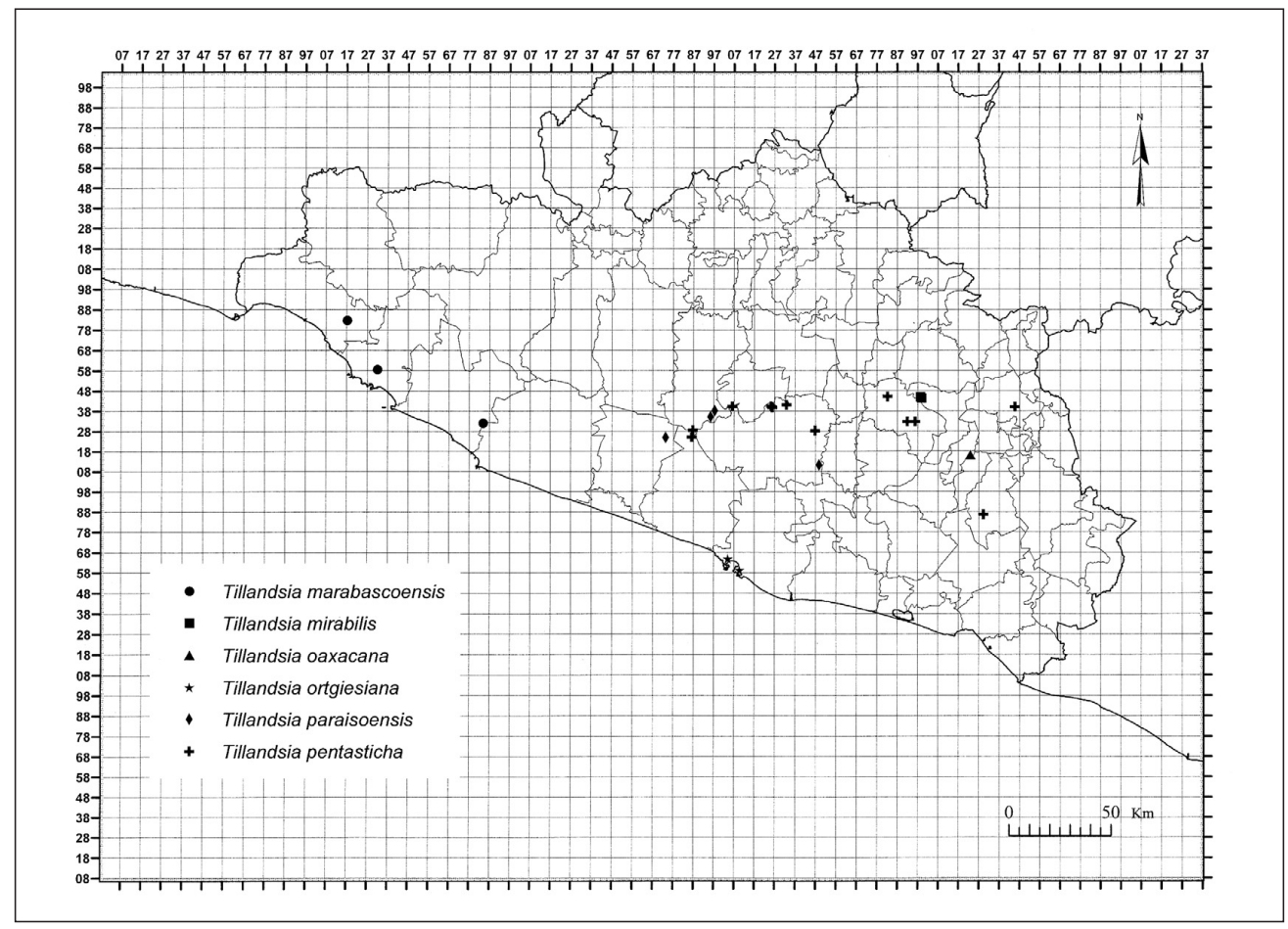

Figura 19. Distribución de algunas especies de Tillandsia en Guerrero.

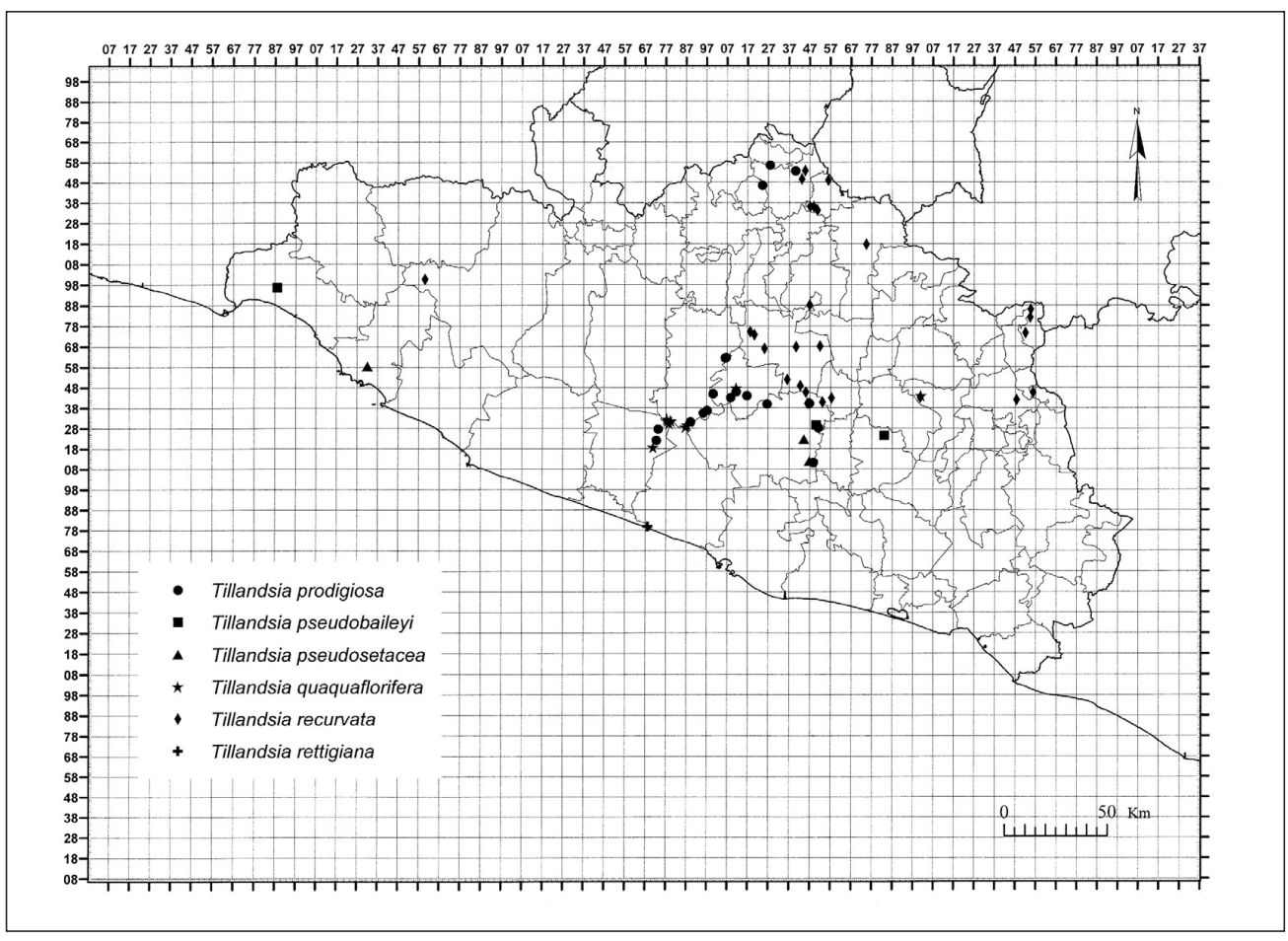

Figura 20. Distribución de algunas especies de Tillandsia en Guerrero. 
FLORA BROMELIOLÓGICA DE GUERRERO

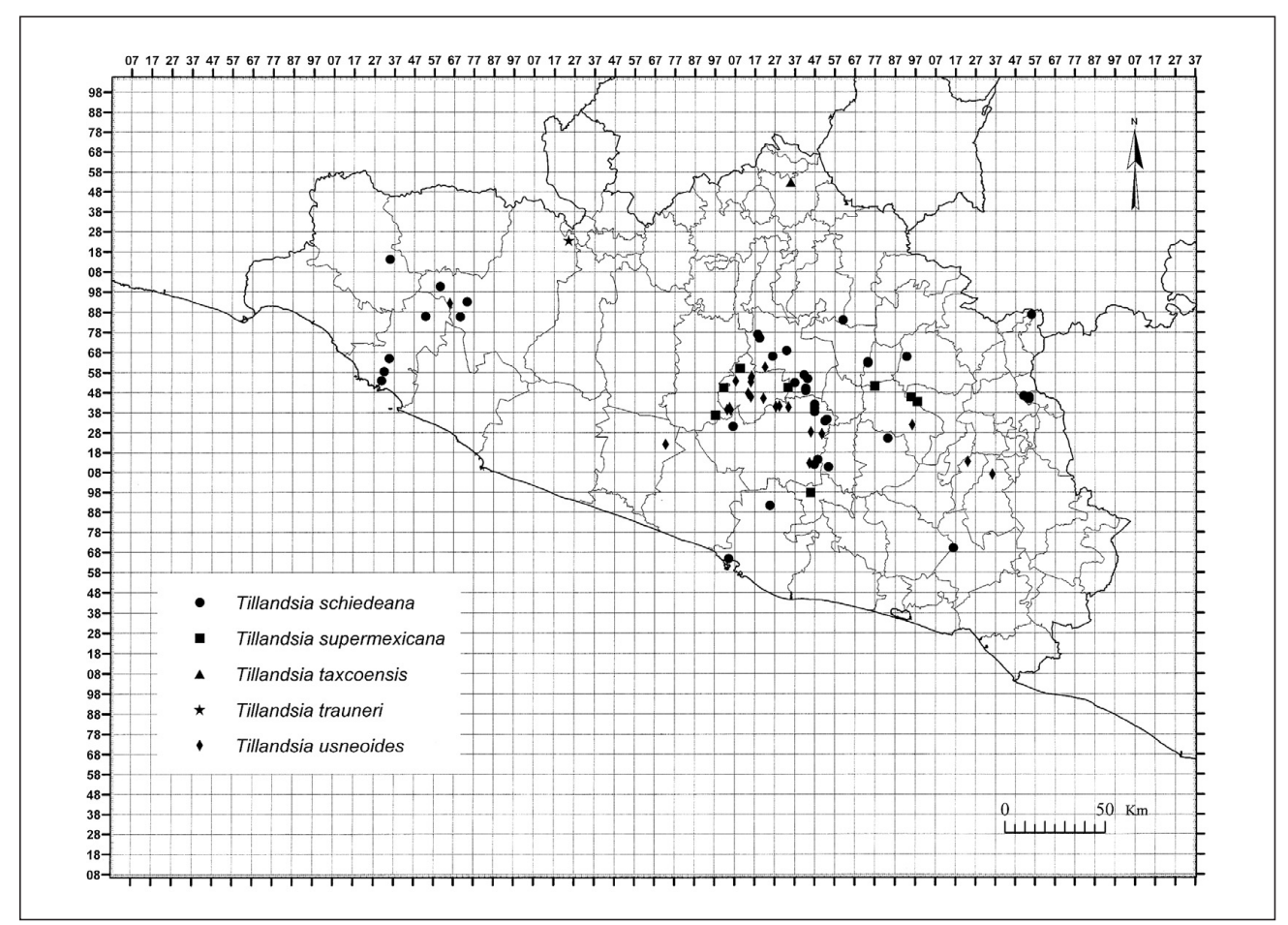

Figura 21. Distribución de algunas especies de Tillandsia en Guerrero.

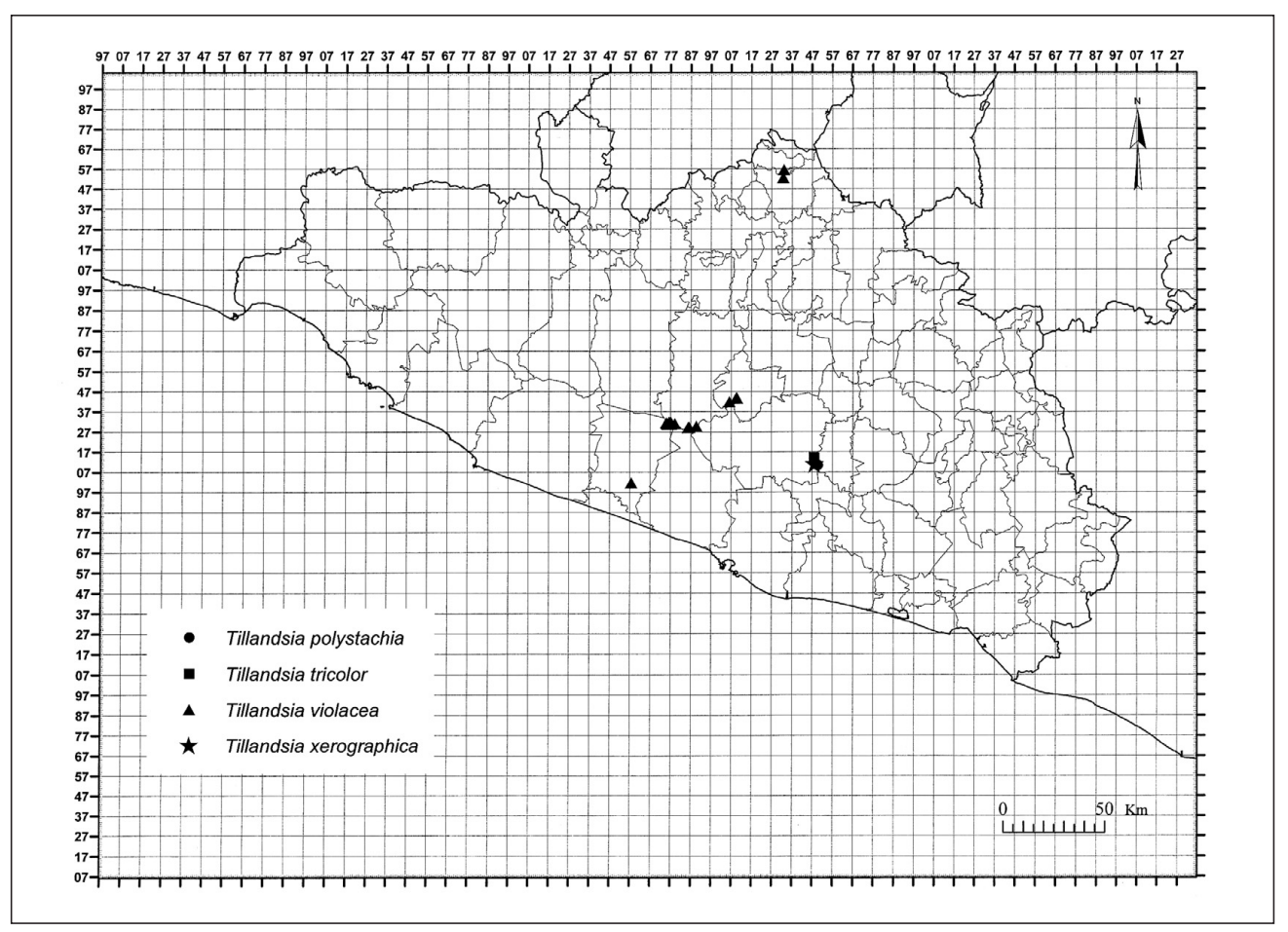

Figura 22. Distribución de algunas especies de Tillandsia en Guerrero. 
Valeria Angélica Pulido-Esparza, Ana Rosa LóPez-Ferrari y Adolfo EsPejo-Serna

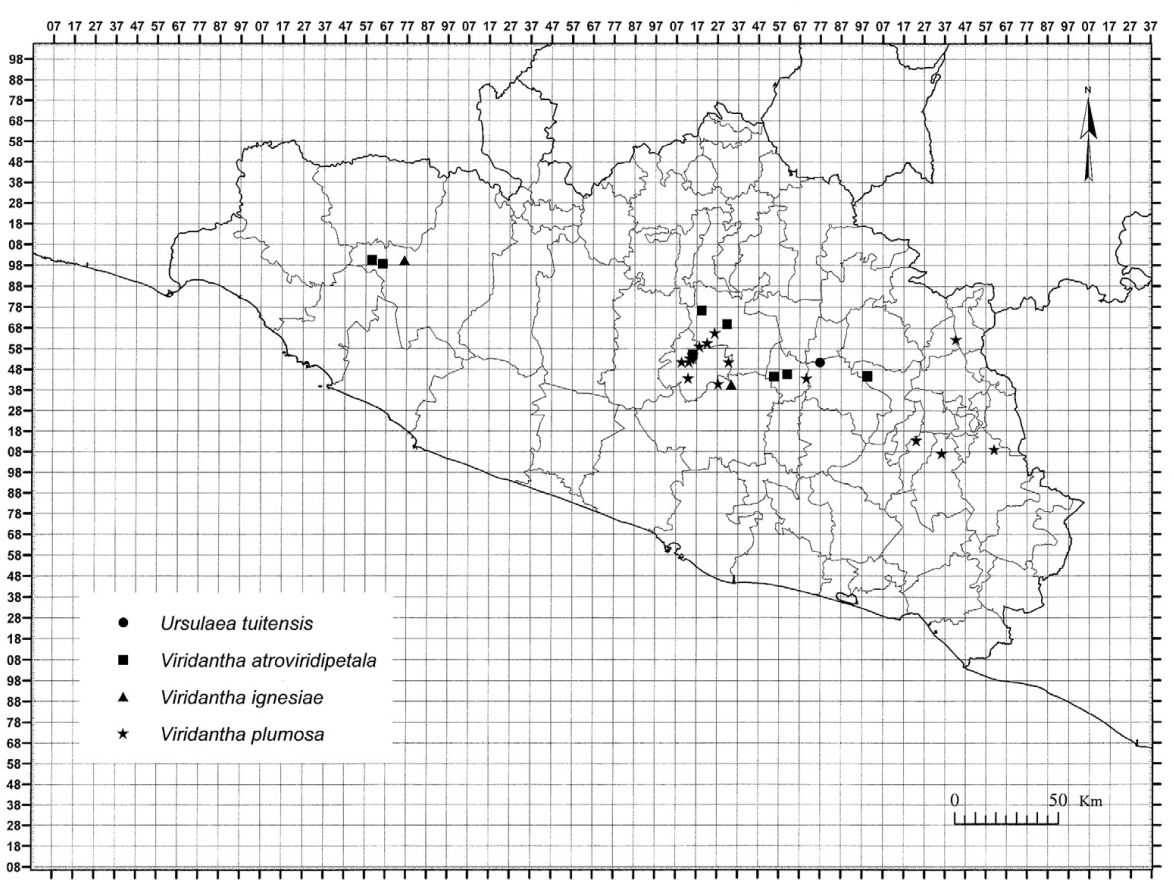

Figura 23. Distribución de las especies de Ursulaea y Viridantha en Guerrero. 